\title{
Analytical solution of the tokamak equilibrium. I. The fixed-boundary case
}

\author{
G. O. Ludwig ${ }^{\text {a) }}$ \\ Departamento de Física, Instituto Tecnológico de Aeronáutica, Comando da Aeronáutica, 12228-900 São José \\ dos Campos, SP, Brazil
}

(Received 16 May 2017; accepted 4 July 2017; published online 10 August 2017)

\begin{abstract}
This article presents an analytical method to evaluate the equilibrium of plasmas in axisymmetric, nested magnetic configurations relevant for tokamak experiments. The emphasis is in the use of spectral representations of the magnetic flux surfaces and variational methods to solve the GradShafranov equation. Approximate solutions are obtained based on power series expansions of the variational moment equations and sectionally continuous representations of the Fourier amplitudes. The method is applied to an up-down asymmetric configuration of an ITER-like tokamak in a fixed-boundary equilibrium. The free-boundary case is treated in the Paper II [G. O. Ludwig, Phys. Plasma 24, 092503 (2017)]. Published by AIP Publishing. [http://dx.doi.org/10.1063/1.4997793]
\end{abstract}

\section{INTRODUCTION}

The analytical description of nested magnetic flux surfaces for axisymmetric configurations is an important tool in the study of the equilibrium, stability, and control of present and future tokamak experiments. The analytical models should represent configurations with an arbitrary aspect ratio, describing the noncircular geometry as well as the possible vertical asymmetry of the flux surfaces. Moreover, the modeled equilibria must constitute adequate solutions of the Grad-Shafranov equation (properly named the ShafranovLüst-Schlüter-Grad-Rubin equation ${ }^{2-4}$ ) and be consistent with externally applied magnetic fields. This problem has been dealt with since the beginning of fusion efforts, but remains open to improvements.

Usually, the fully analytical solutions of the GradShafranov equation assume a simple linear or quadratic dependence of the source term on the poloidal flux function. ${ }^{5-14}$ Taking a different approach, in the present article no assumption is made about the form of the toroidal current density in relation to the poloidal flux, but the flux surfaces are described by appropriate spectral expansions in flux coordinates. Approximate equilibrium solutions are attempted based on a variational moment method and radial power series expansions around the magnetic axis. These solutions are extended from the plasma core to the border using sectionally continuous approximations for the Fourier amplitudes. The optimum matching between core and border asymptotic solutions is performed using a direct variational approach based on a stationary state of the internal energy. For given plasma shape and source profiles with a limited number of free parameters, the flux surface geometry is determined from the radial power series expansions of the variational moment equations in a fast, straightforward manner.

The first task in this work is to develop a general spectral representation of the nested flux surfaces. This is accomplished in Sec. II starting with a mapping between cylindrical $(R, Z)$ and flux $(\rho, \theta)$ coordinates. The Fourier coefficients of this mapping must be determined in order to satisfy the Grad-Shafranov equation. With this objective in mind, power

\footnotetext{
${ }^{\text {a)} E-m a i l: ~ g e r s o n . l u d w i g @ i n p e . b r ~}$
}

series expansions of the poloidal flux function around the magnetic axis are carried out in Sec. III, both in cylindrical and flux coordinates. In this way, various geometrical constraints and relationships are obtained, leading to the general form of the Taylor series expansions in the radial coordinate $\rho$ for the Fourier coefficients of the inverse mapping.

The formulation of one-dimensional variational moment solutions of the Grad-Shafranov equation is presented in Sec. IV, taking advantage of the reduction in dimensionality obtained through elimination of the poloidal angle dependence. The definition of various geometric and magnetic coefficients relevant to the tokamak equilibrium problem is reviewed in this section in accordance with the present formulation. The various energy terms that form both the internal energy integral and the action integral for the tokamak equilibrium are detailed. The Lagrangian method applied to the action integral leads to a system of coupled differential Euler equations for the Fourier coefficients, while the stationary value of the internal energy provides an additional constraint to the equilibrium configuration.

In Sec. V, it is shown how to construct sectionally continuous approximations to the Fourier coefficients based on the Taylor series taken both around the magnetic axis and at the plasma boundary. The relationships between the amplitudes of the Fourier coefficients around the magnetic axis are obtained from expansions of the Euler equations, which are equivalent to the Grad-Shafranov equation. The matching between core and border expansions is performed by searching for an extremum value of the internal plasma energy defined earlier in Sec. IV. The Taylor series on the axis of the geometric and magnetic coefficients of the equilibrium equations is presented in Appendix B, while Appendix C presents the related power series expansions of the Euler equations. The higher order terms in these expansions become quite cumbersome, but have a simple algebraic structure that allows fast solution of the equilibrium.

Finally, Sec. VI presents an application of the method to an ITER-like tokamak equilibrium. A slightly up-down asymmetric configuration is chosen in order to make full use of the method. Some concluding remarks are given in Sec. VII. 


\section{SPECTRAL REPRESENTATION OF THE FLUX SURFACES IN A TOKAMAK PLASMA EQUILIBRIUM}

Consider a mapping between cylindrical coordinates $(R$, $Z)$ and flux coordinates $(\rho, \theta)$ in an axisymmetric system. Here, $\rho$ denotes the radial variable and $\theta$ is the poloidal angle variable. The toroidal angle variable $\phi$ in the cylindrical system corresponds to the toroidal angle $-\zeta$ in the flux coordinates, so that $(R, \phi, Z)$ and $(\rho, \theta, \zeta)$ form right-handed systems. The mapping of a simply connected flux surface in the $(R, Z)$ plane onto a unit circle $z=\exp (i \theta)$ in the complex $z$ plane can be written as ${ }^{15,16}$

$$
\begin{aligned}
\frac{R(\rho, \theta)}{a}+i \frac{Z(\rho, \theta)}{e(\rho) a}= & S_{0}(\rho)+i A_{0}(\rho)+\frac{S_{1}(\rho)+i A_{1}(\rho)}{z^{*}} \\
& +\sum_{n=2}^{\infty}\left(\frac{\rho}{a}\right)^{n-1}\left(S_{n}(\rho)+i A_{n}(\rho)\right)\left(z^{* n}-1\right),
\end{aligned}
$$

where $z^{*}$ denotes the complex conjugate, $a$ is a normalizing radius (minor radius), and $e(\rho), S(\rho)$ and $A(\rho)$ are dimensionless, real differentiable functions. This is a unique representation according to the Riemann mapping theorem. Strictly speaking, the conformal mapping requires all powers of $n$ up to infinity. However, in what follows the expressions of $R(\rho, \theta)$ and $Z(\rho, \theta)$ are taken as a truncated Fourier series which represent the flux surfaces with $2 \leqslant n \leqslant N$ an integer. Using $z^{n}=\cos (n \theta)+i \sin (n \theta)$, the real and imaginary parts of the above expression yield the radial representation

$$
\begin{aligned}
\frac{R(\rho, \theta)}{a}= & S_{0}(\rho)+S_{1}(\rho) \cos \theta-A_{1}(\rho) \sin \theta-\sum_{n=2}^{N}\left(\frac{\rho}{a}\right)^{n-1} \\
& \times\left[S_{n}(\rho)(1-\cos (n \theta))-A_{n}(\rho) \sin (n \theta)\right]
\end{aligned}
$$

and the axial representation

$$
\begin{aligned}
\frac{Z(\rho, \theta)}{e(\rho) a}= & A_{0}(\rho)+A_{1}(\rho) \cos \theta+S_{1}(\rho) \sin \theta-\sum_{n=2}^{N}\left(\frac{\rho}{a}\right)^{n-1} \\
& \times\left[A_{n}(\rho)(1-\cos (n \theta))+S_{n}(\rho) \sin (n \theta)\right] .
\end{aligned}
$$

A set of coupled differential equations, which allow us to determine all the real Fourier coefficients consistent with a tokamak equilibrium, up to a desired order, is derived in Sec. IV using a variational formulation of the Grad-Shafranov equation. The lowest order Fourier coefficients have simple geometrical interpretations, as shown in the following. The origin $\theta=0$ is defined by the point

$$
\begin{aligned}
& \frac{R(\rho, 0)}{a}=S_{0}(\rho)+S_{1}(\rho)=\frac{R_{0}(\rho)+\rho}{a}, \\
& \frac{Z(\rho, 0)}{a}=e(\rho)\left(A_{0}(\rho)+A_{1}(\rho)\right)=\frac{Z_{0}(\rho)}{a},
\end{aligned}
$$

and the opposite point at $\theta=\pi$ by

$$
\begin{aligned}
\frac{R(\rho, \pi)}{a}= & S_{0}(\rho)-S_{1}(\rho)-\sum_{n=2}^{N}\left(\frac{\rho}{a}\right)^{n-1} S_{n}(\rho)\left(1-(-1)^{n}\right) \\
= & \frac{R_{0}(\rho)-\rho}{a}, \\
\frac{Z(\rho, \pi)}{a}= & e(\rho)\left(A_{0}(\rho)-A_{1}(\rho)\right. \\
& \left.-\sum_{n=2}^{N}\left(\frac{\rho}{a}\right)^{n-1} A_{n}(\rho)\left(1-(-1)^{n}\right)\right)=\frac{Z_{0}(\rho)}{a},
\end{aligned}
$$

where the pair $R_{0}(\rho), Z_{0}(\rho)$ corresponds to the position of the major axis. These equations can be solved for $S_{0}(\rho)$, $S_{1}(\rho), A_{0}(\rho)$ and $A_{1}(\rho)$ in terms of $\rho, R_{0}(\rho), Z_{0}(\rho), e(\rho)$ and the higher order coefficients $S_{n}(\rho), A_{n}(\rho)$. Substitution of the results in the expressions for $R(\rho, \theta)$ and $Z(\rho, \theta)$ gives

$$
\begin{aligned}
\frac{R(\rho, \theta)}{a}= & \frac{R_{0}(\rho)}{a}+\frac{\rho}{a} \cos \theta+\sum_{n=1}^{[(N-1) / 2]}\left(\frac{\rho}{a}\right)^{2 n} \\
& \times\left[S_{2 n+1}(\rho)(1-\cos \theta)+A_{2 n+1}(\rho) \sin \theta\right] \\
& -\sum_{n=2}^{N}\left(\frac{\rho}{a}\right)^{n-1}\left[S_{n}(\rho)(1-\cos (n \theta))-A_{n}(\rho) \sin (n \theta)\right], \\
\frac{Z(\rho, \theta)}{e(\rho) a}= & \frac{Z_{0}(\rho)}{e(\rho) a}+\left(\frac{\rho}{a} \sin \theta+\sum_{n=1}^{[(N-1) / 2]}\left(\frac{\rho}{a}\right)^{2 n}\right. \\
& \times\left[A_{2 n+1}(\rho)(1-\cos \theta)-S_{2 n+1}(\rho) \sin \theta\right] \\
& \left.-\sum_{n=2}^{N}\left(\frac{\rho}{a}\right)^{n-1}\left[A_{n}(\rho)(1-\cos (n \theta))+S_{n}(\rho) \sin (n \theta)\right]\right),
\end{aligned}
$$

where $[(N-1) / 2]$ is the greatest integer less than or equal to $(N-1) / 2$.

The set $S_{n}(\rho)$ corresponds to the symmetric and the set $A_{n}(\rho)$ to the antisymmetric coefficients with respect to the equatorial plane $Z=Z_{0}(\rho)$; the elongation coefficient $e(\rho)$ stretches the flux surfaces along the $Z$ coordinate; the value $\rho=0$ corresponds to the position of the magnetic axis

$$
\begin{aligned}
& R(0, \theta)=R_{0}(0)=R_{m}, \\
& Z(0, \theta)=Z_{0}(0)=Z_{m},
\end{aligned}
$$

and $\rho=a$ corresponds to the outermost flux surface, i.e., the plasma edge. These spectral representations for $R(\rho, \theta)$ and $Z(\rho, \theta)$ can also be written in terms of Chebyshev polynomials according to

$$
\cos (n \theta)=T_{n}(\mu), \quad \sin (n \theta)=\sqrt{1-\mu^{2}} U_{n-1}(\mu),
$$

where $\mu=\cos \theta$. Note that the representation described in this section corresponds to an O-type configuration of the magnetic field.

The elongation $\kappa(\rho)$ and the triangularity $\delta(\rho)$ at the extreme points in the $Z$ coordinate are defined by 


$$
\begin{aligned}
& \kappa_{1}(\rho)=\frac{Z\left(\rho, \theta_{1}\right)-Z_{0}(\rho)}{\rho}=\frac{Z_{1}(\rho)-Z_{0}(\rho)}{\rho}, \\
& \delta_{1}(\rho)=\frac{R\left(\rho, \theta_{1}\right)-R_{0}(\rho)}{\rho}=\frac{R_{1}(\rho)-R_{0}(\rho)}{\rho}, \\
& \kappa_{2}(\rho)=\frac{Z\left(\rho, \theta_{2}\right)-Z_{0}(\rho)}{\rho}=\frac{Z_{2}(\rho)-Z_{0}(\rho)}{\rho}, \\
& \delta_{2}(\rho)=\frac{R\left(\rho, \theta_{2}\right)-R_{0}(\rho)}{\rho}=\frac{R_{2}(\rho)-R_{0}(\rho)}{\rho},
\end{aligned}
$$

where $\theta_{1}$ and $\theta_{2}$ are roots of the equation $\partial Z(\rho, \theta) / \partial \theta=0$ which correspond to the extreme upper and lower values of $Z(\rho, \theta)$, respectively. The set of seven parameters $a, R_{0}(a)$,
$Z_{0}(a), \kappa_{1}(a), \delta_{1}(a), \kappa_{2}(a)$, and $\delta_{2}(a)$ at the plasma edge should be sufficient to describe the equilibrium configuration of current tokamak experiments, since the plasma boundary usually presents a simple convex shape. Assuming $\kappa_{2}(a)$ $=\kappa_{1}(a)$ and $\delta_{2}(a)=\delta_{1}(a)$, one has a symmetric configuration with respect to the equatorial plane $Z=Z_{0}(a)$, reducing the modeling of the plasma shape to four parameters besides the minor radius $a$. Even this reduced set of coefficients should be sufficient for most practical purposes. ${ }^{17}$

The surface $S_{T}(\rho)$ of the poloidal cross section of a given flux surface and the volume $V(\rho)$ contained by it can be calculated by integration

$$
\begin{aligned}
S_{T}(\rho)= & \int_{0}^{2 \pi} R(\rho, \theta) \frac{\partial Z(\rho, \theta)}{\partial \theta} d \theta=\pi a^{2} e(\rho)\left(S_{1}^{2}(\rho)+A_{1}^{2}(\rho)-\sum_{n=2}^{N} n\left(\frac{\rho}{a}\right)^{2 n-2}\left[S_{n}^{2}(\rho)+A_{n}^{2}(\rho)\right]\right), \\
V(\rho)= & \pi \int_{0}^{2 \pi} R^{2}(\rho, \theta) \frac{\partial Z(\rho, \theta)}{\partial \theta} d \theta=2 \pi a\left(S_{0}(\rho)-\sum_{n=2}^{N}\left(\frac{\rho}{a}\right)^{n-1} S_{n}(\rho)\right) S_{T}(\rho) \\
& -\frac{\pi^{2}}{2} a^{3} e(\rho) \sum_{n=2}^{N-1}\left(\frac{\rho}{a}\right)^{2 n-3} S_{n}(\rho)\left(4 n\left(\frac{\rho}{a}\right)^{2}\left[S_{1}(\rho) S_{n+1}(\rho)-A_{1}(\rho) A_{n+1}(\rho)\right]\right. \\
& \left.+\sum_{m=2}^{N-n}(3 n+m)\left(\frac{\rho}{a}\right)^{2 m}\left[S_{m}(\rho) S_{n+m}(\rho)+A_{m}(\rho) A_{n+m}(\rho)\right]\right)-\frac{\pi^{2}}{2} a^{3} e(\rho) \sum_{n=2}^{N-1}\left(\frac{\rho}{a}\right)^{2 n-3} A_{n}(\rho) \\
& \times\left(4 n\left(\frac{\rho}{a}\right)^{2}\left[S_{1}(\rho) A_{n+1}(\rho)+A_{1}(\rho) S_{n+1}(\rho)\right]+\sum_{m=2}^{N-n}(3 n+m)\left(\frac{\rho}{a}\right)^{2 m}\left[S_{m}(\rho) A_{n+m}(\rho)-A_{m}(\rho) S_{n+m}(\rho)\right]\right) .
\end{aligned}
$$

The mapping presented in this section corresponds to a simply connected domain. Any doubly connected domain, which is the next simplest case, can be mapped in a conformal way onto an annulus $\left|z_{0}\right|<|z|<1$ with $0<z_{0}<1$ in the complex $z$ plane, but the details and application of this mapping remain to be derived.

\section{CONSISTENT POWER SERIES EXPANSIONS OF THE POLOIDAL FLUX FUNCTION}

In this section, the power series expansion of the normalized poloidal flux function around the magnetic axis is performed both in cylindrical and flux coordinates. The objective here is to obtain adequate Taylor series expansions for the Fourier coefficients which describe the flux surfaces. This work extends the analysis carried out in Ref. 18. As shown below, geometric and algebraic consistency leads to constraints and relationships between the multivariate power series coefficients in cylindrical coordinates and the Fourier coefficients in the spectral representation of the closed flux surfaces. The general form of the Taylor series will be used in Sec. V to obtain approximate solutions of the Euler equations consistent with a Grad-Shafranov equilibrium. The expansion method and the moment cancelation procedure will be demonstrated next keeping up to fourth-order terms, but the final results will be extended to the sixth-order in $\rho$.
The multivariate expansion of the normalized poloidal flux function $\psi=\Phi_{P}(\rho) / \Phi_{P}(a)$ to the fourth-order near the magnetic axis is

$$
\begin{aligned}
\psi(R, Z)= & \psi_{m}+\underbrace{\psi_{m}^{(1,0)}}_{=0}\left(R-R_{m}\right)+\frac{1}{2} \psi_{m}^{(2,0)}\left(R-R_{m}\right)^{2} \\
& +\frac{1}{6} \psi_{m}^{(3,0)}\left(R-R_{m}\right)^{3}+\frac{1}{24} \psi_{m}^{(4,0)}\left(R-R_{m}\right)^{4} \\
& +(\underbrace{\psi_{m}^{(0,1)}}_{=0}+\psi_{m}^{(1,1)}\left(R-R_{m}\right)+\frac{1}{2} \psi_{m}^{(2,1)}\left(R-R_{m}\right)^{2} \\
& \left.+\frac{1}{6} \psi_{m}^{(3,1)}\left(R-R_{m}\right)^{3}\right)\left(Z-Z_{m}\right) \\
& +\left(\frac{1}{2} \psi_{m}^{(0,2)}+\frac{1}{2} \psi_{m}^{(1,2)}\left(R-R_{m}\right)+\frac{1}{4} \psi_{m}^{(2,2)}\left(R-R_{m}\right)^{2}\right) \\
& \times\left(Z-Z_{m}\right)^{2}+\left(\frac{1}{6} \psi_{m}^{(0,3)}+\frac{1}{6} \psi_{m}^{(1,3)}\left(R-R_{m}\right)\right) \\
& \times\left(Z-Z_{m}\right)^{3}+\frac{1}{24} \psi_{m}^{(0,4)}\left(Z-Z_{m}\right)^{4}+\cdots
\end{aligned}
$$

The coefficients $\psi_{m}^{(1,0)}$ and $\psi_{m}^{(0,1)}$ of the linear terms obviously vanish since the flux is proportional to the area, but these conditions can also be determined by first-order terms cancelation as shown in the following. The Fourier 
coefficients can be expanded as a Taylor series in the neighborhood of the magnetic axis $\rho=0$ :

$$
\begin{aligned}
& R_{0}(\rho)=R_{m}+\sum_{k=1}^{\infty} \frac{R_{0}^{(k)}(0)}{k !} \rho^{k} \\
& Z_{0}(\rho)=Z_{m}+\sum_{k=1}^{\infty} \frac{Z_{0}^{(k)}(0)}{k !} \rho^{k} \\
& e(\rho)=\kappa_{m}+\sum_{k=1}^{\infty} \frac{e^{(k)}(0)}{k !} \rho^{k}
\end{aligned}
$$

for the main coefficients, with $\kappa_{m}=e(0)$ denoting the magnetic axis elongation; and

$$
\begin{aligned}
& S_{n}(\rho)=\sum_{k=0}^{\infty} \frac{S_{n}^{(k)}(0)}{k !} \rho^{k}, \\
& A_{n}(\rho)=\sum_{k=0}^{\infty} \frac{A_{n}^{(k)}(0)}{k !} \rho^{k},
\end{aligned}
$$

for the remaining symmetric and antisymmetric coefficients. The values $R_{0}(0)=R_{m}$ and $Z_{0}(0)=Z_{m}$ immediately give $\psi\left[R_{0}(0), Z_{0}(0)\right]=\psi_{m}$ as the zero-order constraint. Now substituting the above Taylor series in the spectral representations $R(\rho, \theta), Z(\rho, \theta)$ of Sec. II, collecting terms of the same power in $\rho$ in the expansion of the flux function $\psi(R, Z)$, and canceling moments in $\theta$, one obtains the conditions and relationships listed below for increasing order in $\rho$.

First-order in $\rho$ :

$$
\begin{aligned}
& \psi_{m}^{(1,0)}=0 \\
& \psi_{m}^{(0,1)}=0 .
\end{aligned}
$$

Second-order in $\rho$ :

$$
\begin{array}{ll}
S_{2}(0)=0, & A_{2}(0)=0, \\
R_{0}^{\prime}(0)=0, & Z_{0}^{\prime}(0)=0,
\end{array}
$$

and

$$
\psi_{m}^{(1,1)}=0, \quad \psi_{m}^{(0,2)}=\frac{\psi_{m}^{(2,0)}}{\kappa_{m}^{2}} .
$$

Third-order in $\rho$ :

$$
S_{3}(0)=0, \quad A_{3}(0)=0, \quad e_{0}^{\prime}(0)=0,
$$

and

$$
\begin{aligned}
& \psi_{m}^{(3,0)}=-3 R_{0}^{\prime \prime}(0) \psi_{m}^{(2,0)}, \\
& \psi_{m}^{(2,1)}=-\left(\frac{Z_{0}^{\prime \prime}(0)}{\kappa_{m}}+4 A_{2}^{\prime}(0)\right) \frac{\psi_{m}^{(2,0)}}{\kappa_{m}}, \\
& \psi_{m}^{(1,2)}=-\left[R_{0}^{\prime \prime}(0)-8 S_{2}^{\prime}(0)\right] \frac{\psi_{m}^{(2,0)}}{\kappa_{m}^{2}}, \\
& \psi_{m}^{(0,3)}=-3\left(\frac{Z_{0}^{\prime \prime}(0)}{\kappa_{m}}-4 A_{2}^{\prime}(0)\right) \frac{\psi_{m}^{(2,0)}}{\kappa_{m}^{3}} .
\end{aligned}
$$

Fourth-order in $\rho$ :

$$
\begin{aligned}
& S_{4}(0)=0, \quad A_{4}(0)=0, \quad S_{2}^{\prime \prime}(0)=0 \\
& A_{2}^{\prime \prime}(0)=0, \quad R_{0}^{(3)}(0)=0, \quad Z_{0}^{(3)}(0)=0
\end{aligned}
$$

and

$$
\begin{aligned}
\psi_{m}^{(3,1)}= & 6\left(\left[R_{0}^{\prime \prime}(0)-4 S_{2}^{\prime}(0)\right] \frac{Z_{0}^{\prime \prime}(0)}{\kappa_{m}}+4\left[R_{0}^{\prime \prime}(0)-S_{2}^{\prime}(0)\right] A_{2}^{\prime}(0)\right. \\
& \left.-\frac{4 A_{3}^{\prime}(0)}{a}\right) \frac{\psi_{m}^{(2,0)}}{\kappa_{m}}, \\
\psi_{m}^{(2,2)}= & \frac{\psi_{m}^{(4,0)}}{3 \kappa_{m}^{2}}-2\left[\left[R_{0}^{\prime \prime}(0)-2 S_{2}^{\prime}(0)\right]\left[R_{0}^{\prime \prime}(0)+14 S_{2}^{\prime}(0)\right]\right. \\
& \left.-\left(\frac{Z_{0}^{\prime \prime}(0)}{\kappa_{m}}-2 A_{2}^{\prime}(0)\right)^{2}+\frac{e^{\prime \prime}(0)}{\kappa_{m}}-\frac{16 S_{3}^{\prime}(0)}{a}\right] \frac{\psi_{m}^{(2,0)}}{\kappa_{m}^{2}} \\
\psi_{m}^{(1,3)}= & 6\left[\left[R_{0}^{\prime \prime}(0)-8 S_{2}^{\prime}(0)\right] \frac{Z_{0}^{\prime \prime}(0)}{\kappa_{m}}\right. \\
& \left.+4\left(3 S_{2}^{\prime}(0) A_{2}^{\prime}(0)+\frac{A_{3}^{\prime}(0)}{a}\right)\right] \frac{\psi_{m}^{(2,0)}}{\kappa_{m}^{3}}, \\
\psi_{m}^{(0,4)}= & \frac{\psi_{m}^{(4,0)}}{\kappa_{m}^{4}}-12\left[\left[R_{0}^{\prime \prime}(0)-2 S_{2}^{\prime}(0)\right]\left[R_{0}^{\prime \prime}(0)+6 S_{2}^{\prime}(0)\right]\right. \\
& \left.-\left(\frac{Z_{0}^{\prime \prime}(0)}{\kappa_{m}}-10 A_{2}^{\prime}(0)\right)\left(\frac{Z_{0}^{\prime \prime}(0)}{\kappa_{m}}-2 A_{2}^{\prime}(0)\right)+\frac{e^{\prime \prime}(0)}{\kappa_{m}}\right] \\
&
\end{aligned}
$$

These relationships become increasingly cumbersome. But one easily verifies that the Fourier coefficients have the general Taylor series expansions:

$$
\begin{aligned}
& R_{0}(\rho)=R_{m}+\sum_{k=1}^{\infty} \frac{R_{0}^{(2 k)}(0)}{(2 k) !} \rho^{2 k}, \\
& Z_{0}(\rho)=Z_{m}+\sum_{k=1}^{\infty} \frac{Z_{0}^{(2 k)}(0)}{(2 k) !} \rho^{2 k}, \\
& e(\rho)=\kappa_{m}+\sum_{k=1}^{\infty} \frac{e^{(2 k)}(0)}{(2 k) !} \rho^{2 k},
\end{aligned}
$$

of even powers in $\rho$ for the main coefficients; and

$$
\begin{aligned}
& S_{n}(\rho)=\sum_{k=1}^{\infty} \frac{S_{n}^{(2 k-1)}(0)}{(2 k-1) !} \rho^{2 k-1}, \\
& A_{n}(\rho)=\sum_{k=1}^{\infty} \frac{A_{n}^{(2 k-1)}(0)}{(2 k-1) !} \rho^{2 k-1},
\end{aligned}
$$

of odd powers in $\rho$ for the remaining coefficients.

The flux function can be written as a Taylor series in $\rho$

$$
\psi(\rho)=\psi_{m}+\frac{\rho^{2}}{2 !} \psi^{\prime \prime}(0)+\frac{\rho^{4}}{4 !} \psi^{(4)}(0)+\frac{\rho^{6}}{6 !} \psi^{(6)}(0)+\cdots
$$

where the coefficients up to the sixth-order are given by: 


$$
\begin{aligned}
\psi^{\prime \prime}(0)= & \psi_{m}^{(2,0)} \\
\psi^{(4)}(0)= & \psi_{m}^{(4,0)}-3\left[5 R_{0}^{\prime \prime}(0)^{2}+\left(\frac{Z_{0}^{\prime \prime}(0)}{\kappa_{m}}+8 A_{2}^{\prime}(0)\right) \frac{Z_{0}^{\prime \prime}(0)}{\kappa_{m}}\right] \psi_{m}^{(2,0)} \\
\psi^{(6)}(0)= & \psi_{m}^{(6,0)}-15\left[7 R_{0}^{\prime \prime}(0)^{2}+\left(\frac{Z_{0}^{\prime \prime}(0)}{\kappa_{m}}+8 A_{2}^{\prime}(0)\right) \frac{Z_{0}^{\prime \prime}(0)}{\kappa_{m}}\right] \psi_{m}^{(4,0)} \\
& -15\left\{7\left[R_{0}^{(4)}(0)-6 R_{0}^{\prime \prime}(0)^{3}\right] R_{0}^{\prime \prime}(0)+\frac{Z_{0}^{(4)}(0)}{\kappa_{m}}\left(\frac{Z_{0}^{\prime \prime}(0)}{\kappa_{m}}+4 A_{2}^{\prime}(0)\right)\right. \\
& +6\left[R_{0}^{\prime \prime}(0)\left[R_{0}^{\prime \prime}(0)-28 S_{2}^{\prime}(0)\right]-\frac{e^{\prime \prime}(0)}{\kappa_{m}}+4\left(7 S_{2}^{\prime}(0)^{2}+\frac{4 S_{3}^{\prime}(0)}{a}\right)-4\left(\frac{Z_{0}^{\prime \prime}(0)}{\kappa_{m}}+3 A_{2}^{\prime}(0)\right) A_{2}^{\prime}(0)\right]\left(\frac{Z_{0}^{\prime \prime}(0)}{\kappa_{m}}\right)^{2} \\
& +8\left[3\left(2 R_{0}^{\prime \prime}(0)\left[R_{0}^{\prime \prime}(0)-7 S_{2}^{\prime}(0)\right]-\frac{e^{\prime \prime}(0)}{\kappa_{m}}+8 S_{2}^{\prime}(0)^{2}+\frac{8 S_{3}^{\prime}(0)}{a}\right) A_{2}^{\prime}(0)-6\left[7 R_{0}^{\prime \prime}(0)-4 S_{2}^{\prime}(0)\right]\right. \\
& \left.\left.\frac{A_{3}^{\prime}(0)}{a}+A_{2}^{(3)}(0)+\frac{12 A_{4}^{\prime}(0)}{a^{2}}\right] \frac{Z_{0}^{\prime \prime}(0)}{\kappa_{m}}\right\} \psi_{m}^{(2,0)} .
\end{aligned}
$$

This series for $\psi(\rho)$ takes the form of a "multipolar" expansion. Coupled with $\psi(R, Z)$ it shows that the Fourier coefficients of the spectral representation map the multivariate derivatives $\psi_{m}^{(2,0)}, \psi_{m}^{(4,0)}, \psi_{m}^{(6,0)} \ldots$, taken along the $R$ axis, onto any point of the $(R, Z)$ plane. The Fourier coefficients describe the geometry of the flux surfaces, while the multivariate derivatives along the $R$ axis are directly related to the toroidal current profile, which composes the internal source term in the Grad-Shafranov equation. The flux function $\psi(\rho)$ is an even function of $\rho$, since it varies with the area of the poloidal cross section, which can be expanded as:

$$
\begin{aligned}
\frac{S_{T}(\rho)}{\pi \kappa_{m} \rho^{2}}= & 1+\frac{\rho^{2}}{2}\left[\frac{e^{\prime \prime}(0)}{\kappa_{m}}-4\left(S_{2}^{\prime}(0)^{2}+A_{2}^{\prime}(0)^{2}+\frac{S_{3}^{\prime}(0)}{a}\right)\right] \\
& +\frac{\rho^{4}}{24}\left[\frac{e^{(4)}(0)}{\kappa_{m}}-24 \frac{e^{\prime \prime}(0)}{\kappa_{m}}\left(S_{2}^{\prime}(0)^{2}+A_{2}^{\prime}(0)^{2}+\frac{S_{3}^{\prime}(0)}{a}\right)\right. \\
& -16\left[S_{2}^{\prime}(0) S_{2}^{(3)}(0)+A_{2}^{\prime}(0) A_{2}^{(3)}(0)\right] \\
& \left.-48\left(\frac{S_{3}^{\prime}(0)^{2}}{a^{2}}+\frac{A_{3}^{\prime}(0)^{2}}{a^{2}}\right)-8 \frac{S_{3}^{(3)}(0)}{a}-48 \frac{S_{5}^{\prime}(0)}{a^{3}}\right]+\cdots
\end{aligned}
$$

To be entirely consistent within the sixth-order in $\rho$ one must include pentagonal $S_{4}(\rho), A_{4}(\rho)$ and hexagonal $S_{5}(\rho), A_{5}(\rho)$ corrections, as indicated by the above expansions for $\psi(\rho)$ and $S_{T}(\rho)$. However, as pointed out in Sec. II, it is usually sufficient to describe the equilibrium, besides the normalizing minor radius $a$, by a set of seven parameters $R_{0}(a), Z_{0}(a), e(a), S_{2}(a), A_{2}(a), S_{3}(a)$, and $A_{3}(a)$, which can be determined from the vertical reference position $Z_{0}(a)$ and the fixed points $R_{0}(a)+a, R_{0}(a)-a, Z_{0}(a)+\kappa_{1}(a) a$, $Z_{0}(a)-\kappa_{1}(a) a, R_{0}(a)-\delta_{1}(a) a$, and $R_{0}(a)-\delta_{2}(a) a$, defined on the plasma boundary. Thus, the representation of the flux surfaces in the remainder of this article will be mostly limited to Shafranov shift, elongation, triangularity, and quadrangularity effects, keeping the up-down asymmetry. Higher order corrections in the plasma shape require very high precision in the calculations and are practically impossible to detect experimentally in a tokamak configuration.

\section{SIMPLIFIED VARIATIONAL MOMENT SOLUTION TO THE TOKAMAK PLASMA EQUILIBRIUM PROBLEM}

In this section, the definitions and formulas leading to a variational moment solution of the Grad-Shafranov equation are reviewed. The formulation follows previous works ${ }^{16,19}$ and reduces the problem to a set of ordinary differential equations in the radial variable $\rho$, maintaining the original concept of the variational moment method. ${ }^{18}$ The variational method provides a set of coupled differential Euler equations for the Fourier coefficients of the mapping between cylindrical and flux coordinates, while the stationary value of the internal energy gives an additional constraint to the equilibrium configuration.

The flux function $\Phi=\Phi_{0}-\Phi_{P}$ between the symmetry axis and a given flux surface is related to the toroidal current density $j_{T}$ by Ampère's law in flux coordinates

$$
\begin{aligned}
\Delta^{2} \Phi & =h_{\zeta}^{2} \nabla \cdot\left(h_{\zeta}^{-2} \nabla \Phi\right) \\
& =\frac{h_{\zeta}^{2}}{\sqrt{g}}\left[\frac{\partial}{\partial \rho}\left(\frac{h_{\theta}^{2}}{\sqrt{g}} \frac{d \Phi}{d \rho}\right)-\frac{d \Phi}{d \rho} \frac{\partial}{\partial \theta}\left(\frac{g_{\rho \theta}}{\sqrt{g}}\right)\right]=-2 \pi \mu_{0} h_{\zeta} j_{T} .
\end{aligned}
$$

In terms of the spectral representations in cylindrical coordinates (transformation to flux coordinates $(R, \phi, Z) \rightarrow(\rho, \theta, \zeta)$ ), the metric coefficients are given by

$$
\begin{aligned}
& g_{\rho \rho}=h_{\rho}^{2}=\left(\frac{\partial R}{\partial \rho}\right)^{2}+\left(\frac{\partial Z}{\partial \rho}\right)^{2}, \\
& g_{\theta \theta}=h_{\theta}^{2}=\left(\frac{\partial R}{\partial \theta}\right)^{2}+\left(\frac{\partial Z}{\partial \theta}\right)^{2}, \\
& g_{\xi \xi}=h_{\zeta}^{2}=R^{2}, \\
& g_{\rho \theta}=\frac{\partial R}{\partial \rho} \frac{\partial R}{\partial \theta}+\frac{\partial Z}{\partial \rho} \frac{\partial Z}{\partial \theta},
\end{aligned}
$$


and the Jacobian by

$$
\sqrt{g}=\sqrt{h_{\zeta}^{2}\left(h_{\rho}^{2} h_{\theta}^{2}-g_{\rho \theta}^{2}\right)}=R\left(\frac{\partial R}{\partial \rho} \frac{\partial Z}{\partial \theta}-\frac{\partial R}{\partial \theta} \frac{\partial Z}{\partial \rho}\right) .
$$

The metric coefficients can be easily calculated from the spectral representations given in Secs. II and III. The toroidal current density $j_{T}$ represents both the internal and external sources with respect to the flux surface. Note that the total poloidal flux $\Phi=\Phi_{0}-\Phi_{P}$ is given by the difference between the flux $\Phi_{0}$ linked by the magnetic axis and the poloidal flux $\Phi_{P}$, between the magnetic axis and the flux surface, which is produced by the toroidal plasma current.

Using magnetic flux coordinates $(\rho, \theta, \zeta)$, the internal toroidal current density in a single-nested axisymmetric tokamak plasma equilibrium is given by

$$
\begin{aligned}
j_{T}(\rho, \theta)= & \frac{\mu_{0} d I_{T} / d \rho}{2 \pi h_{\zeta}(\rho, \theta) d L / d \rho}-\frac{K(\rho)}{I_{T}(\rho)} \\
& \times\left(2 \pi h_{\zeta}(\rho, \theta)-\frac{\mu_{0} d V / d \rho}{2 \pi h_{\zeta}(\rho, \theta) d L / d \rho}\right) \frac{d p}{d \rho},
\end{aligned}
$$

where $I_{T}(\rho)$ and $p(\rho)$ denote the radial profiles of the toroidal plasma current and plasma pressure, respectively. In this form, Ampère's law leads to the Grad-Shafranov equation. The total toroidal current contained by a flux surface is

$$
I_{T}(\rho)=\iint_{S_{T}(\rho)} j_{T}(\rho, \theta) d^{2} r(\zeta)
$$

where $d^{2} r(\zeta)=\left(\sqrt{g} / h_{\zeta}\right) d \rho d \theta$ is the differential area element in the coordinate surface $\zeta=$ constant and $S_{T}(\rho)$ $=\iint d^{2} r(\zeta)$ is the area of the poloidal cross section. The volume enclosed by a flux surface is

$$
V(\rho)=2 \pi \iint_{S_{T}(\rho)} h_{\zeta}(\rho, \theta) d^{2} r(\zeta) .
$$

Furthermore, the inductance of the toroidal solenoid defined by a flux surface is evaluated by the integral

$$
L(\rho)=\frac{\mu_{0}}{2 \pi} \iint_{S_{T}(\rho)} \frac{d^{2} r(\zeta)}{h_{\zeta}(\rho, \theta)},
$$

and the inverse kernel of the internal inductance of the plasma loop by

$$
K(\rho)=\frac{1}{2 \pi \mu_{0}} \oint \frac{|\nabla \rho|}{h_{\zeta}(\rho, \theta)} d \ell(\theta) .
$$

Here, $d \ell(\theta)=h_{\theta} d \theta$ is the differential arc length along the coordinate-curve $\theta$. The unit vector normal to the flux surfaces is $\hat{n}=\nabla \rho /|\nabla \rho|$, where

$$
|\nabla \rho|=\frac{h_{\theta}(\rho, \theta) h_{\zeta}(\rho, \theta)}{\sqrt{g}(\rho, \theta)} .
$$

The geometric and magnetic equilibrium coefficients can be calculated taking the poloidal-angle average $\langle\ldots\rangle_{\theta}$ $=(2 \pi)^{-1} \int_{0}^{2 \pi}(\ldots) d \theta$ :

$$
\begin{gathered}
\frac{d S_{T}}{d \rho}=2 \pi\left\langle\frac{\sqrt{g}(\rho, \theta)}{h_{\zeta}(\rho, \theta)}\right\rangle_{\theta}, \\
\frac{d V}{d \rho}=4 \pi^{2}\langle\sqrt{g}(\rho, \theta)\rangle_{\theta}, \\
\frac{d L}{d \rho}=\mu_{0}\left\langle\frac{\sqrt{g}(\rho, \theta)}{h_{\zeta}^{2}(\rho, \theta)}\right\rangle_{\theta}, \\
K(\rho)=\frac{1}{\mu_{0}}\left\langle\frac{h_{\theta}^{2}(\rho, \theta)}{\sqrt{g}(\rho, \theta)}\right\rangle_{\theta} .
\end{gathered}
$$

Hence, after integration by parts (the expressions for $S_{T}(\rho)$ and $V(\rho)$ were already introduced in Sec. II):

$$
\begin{aligned}
S_{T}(\rho) & =\int_{0}^{2 \pi}\left(R(\rho, \theta) \frac{\partial Z}{\partial \theta}\right) d \theta, \\
V(\rho) & =\pi \int_{0}^{2 \pi}\left(R^{2}(\rho, \theta) \frac{\partial Z}{\partial \theta}\right) d \theta, \\
L(\rho) & =-\frac{\mu_{0}}{2 \pi} \int_{0}^{2 \pi}\left(\frac{Z(\rho, \theta)}{R(\rho, \theta)} \frac{\partial R}{\partial \theta}\right) d \theta, \\
K(\rho) & =\frac{1}{2 \pi \mu_{0}} \int_{0}^{2 \pi}\left(\frac{h_{\theta}^{2}(\rho, \theta)}{\sqrt{g}(\rho, \theta)}\right) d \theta .
\end{aligned}
$$

Note that the axisymmetric flux-surface average is related to the poloidal-angle average:

$$
\langle\ldots\rangle=\frac{4 \pi^{2}}{d V / d \rho}\langle\sqrt{g}(\rho, \theta)(\ldots)\rangle_{\theta} .
$$

Using a spectral representation for the flux surfaces as presented in Sec. II, the $\theta$-integrals can be calculated by direct integration or by the method of residues, ${ }^{16}$ effectively reducing the problem to a one-dimensional dependence of the Fourier coefficients on $\rho$. As an example, the geometric coefficients $S_{T}(\rho)$ and $V(\rho)$ were calculated in Sec. II. But the analytical results are convenient only in simple or limiting cases. ${ }^{19}$ In general, the above poloidal-angle averages can be numerically calculated by Gauss-Chebyshev quadrature with adequate precision using few terms $(n \geqslant 2 N$, where $N$ is the Fourier series order):

$$
\frac{1}{2 \pi} \int_{0}^{2 \pi} f(\theta) d \theta \cong \frac{1}{2 n} \sum_{k=1}^{n}\left[f\left(\theta_{k}\right)+f\left(\pi+\theta_{k}\right)\right] .
$$

Here, $\theta_{k}=(2 k-1) \pi /(2 n)$, which corresponds to an optimum integration mesh in the poloidal direction. For symmetric integrands, this reduces to

$$
\frac{1}{\pi} \int_{0}^{\pi} f^{(s)}(\theta) d \theta \cong \frac{1}{n} \sum_{k=1}^{n} f^{(s)}\left(\theta_{k}\right) .
$$

The flux-surface averaged Grad-Shafranov equation (equilibrium equation) for the poloidal flux function $\Phi_{P}$ is ${ }^{20}$ 


$$
\frac{d \Phi_{P}}{d \rho} \frac{d}{d \rho}\left(K(\rho) \frac{d \Phi_{P}}{d \rho}\right)+\frac{d V}{d \rho} \frac{d p}{d \rho}+\frac{1}{2} \frac{d L}{d \rho} \frac{d I^{2}}{d \rho}=0 .
$$

This equation for the tokamak equilibrium can be obtained by variational principles from the various stored energy terms, which are presented in the following paragraphs. The poloidal, $\Phi_{P}$, and toroidal, $\Phi_{T}$, flux functions satisfy the firstorder differential relationships:

$$
\begin{aligned}
& \frac{d \Phi_{P}}{d \rho}=\frac{I_{T}(\rho)}{K(\rho)}, \\
& \frac{d \Phi_{T}}{d \rho}=I(\rho) \frac{d L}{d \rho} .
\end{aligned}
$$

Here, $I(\rho)$ is the total poloidal current due to the plasma and toroidal field coils, and external to the magnetic surface. The poloidal plasma current is $I_{P}(\rho)=I(0)-I(\rho)$. The total poloidal current can be calculated in terms of $p(\rho)$ and $I_{T}(\rho)$ using the equilibrium equation (40)

$$
\begin{aligned}
I^{2}(\rho) & =I^{2}(0)-\int_{0}^{\rho} \frac{2}{d L / d \rho^{\prime}}\left(\frac{1}{2 K\left(\rho^{\prime}\right)} \frac{d I_{T}^{2}}{d \rho^{\prime}}+\frac{d V}{d \rho^{\prime}} \frac{d p}{d \rho^{\prime}}\right) d \rho^{\prime} \\
& =I^{2}(a)+\int_{\rho}^{a} \frac{2}{d L / d \rho^{\prime}}\left(\frac{1}{2 K\left(\rho^{\prime}\right)} \frac{d I_{T}^{2}}{d \rho^{\prime}}+\frac{d V}{d \rho^{\prime}} \frac{d p}{d \rho^{\prime}}\right) d \rho^{\prime}
\end{aligned}
$$

where $\rho=a$ designates the plasma edge, $I(a)=$ $2 \pi R_{0}(a) B_{0} / \mu_{0}$ is the total current in the toroidal field coils (rod current), and $I(0)=2 \pi R_{m} B_{m} / \mu_{0} ; B_{0}$ corresponds to the external toroidal magnetic field at the geometric center $R_{0}(a)$; and $B_{m}$ corresponds to the total magnetic field on the magnetic axis $R_{m}$. The poloidal current density is given in terms of the total poloidal current by

$$
j_{P}(\rho, \theta)=-\frac{h_{\theta}(\rho, \theta)}{2 \pi \sqrt{g}(\rho, \theta)} \frac{d I}{d \rho}=\frac{h_{\theta}(\rho, \theta)}{2 \pi \sqrt{g}(\rho, \theta)} \frac{d I_{P}}{d \rho},
$$

and the safety factor is given by the ratio

$$
q(\rho)=\frac{d \Phi_{T}}{d \Phi_{P}}=\frac{I(\rho)}{I_{T}(\rho)} K(\rho) \frac{d L}{d \rho} .
$$

The magnetic field in a tokamak configuration can be written as

$$
\vec{B}=\frac{1}{2 \pi} \frac{d \Phi_{P}}{d \rho}(\nabla \zeta \times \nabla \rho)+\frac{\mu_{0}}{2 \pi} I(\rho) \nabla \zeta=\vec{B}_{P}+\vec{B}_{T},
$$

where the amplitudes of the poloidal and toroidal field components are

$$
\begin{aligned}
& B_{P}(\rho, \theta)=\frac{\left|\nabla \Phi_{P}\right|}{2 \pi h_{\zeta}(\rho, \theta)}=\frac{h_{\theta}(\rho, \theta)}{2 \pi \sqrt{g}(\rho, \theta)} \frac{d \Phi_{P}}{d \rho}, \\
& B_{T}(\rho, \theta)=\frac{\mu_{0} I(\rho)}{2 \pi h_{\zeta}(\rho, \theta)} .
\end{aligned}
$$

Note that $|\nabla \zeta \times \nabla \rho|=h_{\theta}(\rho, \theta) / \sqrt{g}(\rho, \theta)$ and $|\nabla \zeta|=1 /$ $h_{\zeta}(\rho, \theta)$.
Next, the various energy contributions to the tokamak equilibrium will be derived in detail. This allows us to correctly define both the internal energy $U(a)$, used in the direct variational procedure, and the action functional $J(a)$, which leads to the variational Euler equations for the Fourier coefficients describing the flux surfaces. Both variational approaches will be used in Sec. V to obtain approximate solutions to the Grad-Shafranov equation. These energy expressions are also useful in some applications requiring detailed comparison of energy terms. ${ }^{21}$ First, the internal magnetostatic energy stored in the plasma loop is evaluated by a volume integral with $d^{3} r=\sqrt{g} d \rho d \theta d \zeta$ as

$$
W_{P}(a)=\iiint_{V(a)} \frac{B_{P}^{2}}{2 \mu_{0}} d^{3} r=\int_{0}^{a} \frac{I_{T}^{2}(\rho)}{2 K(\rho)} d \rho=\frac{1}{2} L_{T}(a) I_{T}^{2}(a),
$$

which defines the internal self-inductance $L_{T}(a)$ of the plasma loop. Likewise, the total magnetic energy stored in the plasma solenoid is

$$
\iiint_{V(a)} \frac{B_{T}^{2}}{2 \mu_{0}} d^{3} r=\int_{0}^{a} \frac{I^{2}(\rho)}{2} \frac{d L}{d \rho} d \rho
$$

and the magnetic energy stored inside the empty toroidal plasma solenoid, that is, the energy due to the external field $\vec{B}_{T, 0}$, is

$$
\begin{aligned}
W_{T, 0}(a) & =\iiint_{V(a)} \frac{B_{T, 0}^{2}}{2 \mu_{0}} d^{3} r \\
& =2 \pi \int_{0}^{a} \int_{0}^{2 \pi} \frac{1}{2 \mu_{0}}\left(\frac{B_{0} R_{0}(a)}{h_{\zeta}(\rho, \theta)}\right)^{2} \sqrt{g}(\rho, \theta) d \rho d \theta \\
& =\frac{1}{2} L(a) I^{2}(a),
\end{aligned}
$$

with $L(0)=0$. Hence, the internal magnetostatic energy stored in the plasma solenoid (total energy less the vacuum field energy) is

$$
\begin{aligned}
W_{T}(a) & =\iiint_{V(a)} \frac{B_{T}^{2}-B_{T, 0}^{2}}{2 \mu_{0}} d^{3} r=\int_{0}^{a} \frac{I^{2}(\rho)-I^{2}(a)}{2} \frac{d L}{d \rho} d \rho \\
& =\int_{0}^{a} L(\rho) I(\rho) \frac{d I_{P}}{d \rho} d \rho \\
& =\int_{0}^{a} L(\rho)\left[I(a)+I_{P}(a)-I_{P}(\rho)\right] \frac{d I_{P}}{d \rho} d \rho \\
& =\frac{1}{2} \int_{0}^{a}\left[I_{P}(a)-I_{P}(\rho)\right]^{2} \frac{d L}{d \rho} d \rho+I(a) \int_{0}^{a} L(\rho) \frac{d I_{P}}{d \rho} d \rho .
\end{aligned}
$$

Defining the internal self-inductance of the toroidal plasma solenoid 


$$
L_{P}(a)=\frac{1}{I_{P}^{2}(a)} \int_{0}^{a}\left[I_{P}(a)-I_{P}(\rho)\right]^{2} \frac{d L}{d \rho} d \rho
$$

and the mutual inductance between the toroidal field coils and the toroidal plasma solenoid

$$
L_{M}(a)=\frac{1}{I_{P}(a)} \int_{0}^{a} L(\rho) \frac{d I_{P}}{d \rho} d \rho,
$$

the energy integral $W_{T}(a)$ can be written as

$$
\begin{aligned}
W_{T}(a) & =\iiint_{V(a)} \frac{B_{T}^{2}-B_{T, 0}^{2}}{2 \mu_{0}} d^{3} r \\
& =\frac{1}{2} L_{P}(a) I_{P}^{2}(a)+L_{M}(a) I(a) I_{P}(a) .
\end{aligned}
$$

The tokamak plasma magnetization is defined by

$$
\vec{M}=-\frac{I_{P}(\rho)}{2 \pi} \nabla \zeta
$$

so that

$$
\nabla \times \vec{M}=-\frac{1}{2 \pi} \frac{d I_{P}}{d \rho} \nabla \rho \times \nabla \zeta=\vec{j}_{P}
$$

The integral $W_{M}(a)=L_{M}(a) I(a) I_{P}(a)$ gives the energy required to establish the plasma magnetization in the external magnetic field $\vec{B}_{T, 0}$, not taking into account the work done in creating $\vec{M}$. Assuming $I(a)>0$ the tokamak equilibrium configuration is diamagnetic if $L_{M}(a) I_{P}(a)<0$ and paramagnetic if $L_{M}(a) I_{P}(a)>0$. A detailed calculation of the interaction energy $W_{M}(a)$ between the plasma and the external toroidal magnetic field is presented in Appendix A.

Finally, the energy stored in the plasma due to quasistatic work is

$$
W_{Q S}(a)=\iiint_{V(a)} p d^{3} r=\int_{0}^{a} p(\rho) \frac{d V}{d \rho} d \rho .
$$

Collecting the various energy terms, the internal energy can be written as

$$
\begin{aligned}
U(a)= & \iiint_{V(a)}\left(\frac{B_{P}^{2}}{2 \mu_{0}}+\frac{B_{T}^{2}-B_{T, 0}^{2}}{2 \mu_{0}}+p\right) d^{3} r \\
= & W_{P}(a)+W_{T}(a)+W_{Q S}(a) \\
= & \frac{1}{2} L_{T}(a) I_{T}^{2}(a)+\frac{1}{2} L_{P}(a) I_{P}^{2}(a) \\
& +L_{M}(a) I(a) I_{P}(a)+W_{Q S}(a) .
\end{aligned}
$$

The energy $U(a)$ is the change in energy within the volume $V(a)$ in the external field when the plasma is introduced; it excludes the energy $W_{T, 0}(a)=L(a) I^{2}(a) / 2$ of the volume prior to the introduction of the plasma. In terms of the equilibrium variables

$$
\begin{aligned}
U(a)= & \int_{0}^{a}\left[\left(I_{T}(\rho)+\frac{2 L(\rho)}{d L / d \rho} \frac{d I_{T}}{d \rho}\right) \frac{I_{T}(\rho)}{2 K(\rho)}\right. \\
& \left.+\left(p(\rho)+\frac{L(\rho)}{d L / d \rho} \frac{d p}{d \rho}\right) \frac{d V}{d \rho}\right] d \rho .
\end{aligned}
$$

The internal energy $U(a)$ is stationary under virtual displacements $\delta \rho$ of the radius $\rho$ for fixed-boundary conditions $\delta \rho(a)=0$. This is an application of Castigliano's principle that allows direct variational solutions to the Grad-Shafranov equation. ${ }^{16}$ Characterization of the toroidal equilibrium of a magnetically confined plasma as a stationary state of the internal energy has been carried out originally by Kruskal and Kulsrud. ${ }^{22}$ In the present formulation, one can identify the term corresponding to the magnetic energy stored in the plasma loop, $W_{P}(a)$, and the terms corresponding to the total electromechanical and quasistatic expansion work done by the toroidal plasma solenoid on its surroundings, $W_{T}(a)+W_{Q S}(a)$ :

$$
\begin{aligned}
W_{P}(a) & =\frac{1}{2} \int_{0}^{a} I_{T}(\rho) d \Phi_{P}, \\
W_{T}(a)+W_{Q S}(a) & =\int_{0}^{a}\left(\frac{I^{2}(\rho)-I^{2}(a)}{2} d L+p(\rho) d V\right) .
\end{aligned}
$$

As it stands, $W_{T}(a)+W_{Q S}(a)$ denotes the positive output work. The input work $-W_{T}(a)-W_{Q S}(a)$ is positive if it increases the internal energy of the system. The sum $W_{P}(a)+W_{T}(a)+W_{Q S}(a)=U(a)$ corresponds to the total internal energy stored by magnetization and adiabatic compression of the plasma ring.

As will be shown next, the equilibrium profiles are such as to give an extremum of the action functional

$$
\begin{aligned}
J(a)= & W_{P}(a)-W_{T}(a)-W_{Q S}(a) \\
= & \iiint_{V(a)}\left(\frac{B_{P}^{2}}{2 \mu_{0}}-\frac{B_{T}^{2}-B_{T, 0}^{2}}{2 \mu_{0}}-p\right) d^{3} r \\
= & \int_{0}^{a}\left[\left(I_{T}(\rho)-\frac{2 L(\rho)}{d L / d \rho} \frac{d I_{T}}{d \rho}\right) \frac{I_{T}(\rho)}{2 K(\rho)}\right. \\
& \left.+\left(V(\rho)-\frac{L(\rho)}{d L / d \rho} \frac{d V}{d \rho}\right) \frac{d p}{d \rho}\right] d \rho \\
= & \int_{0}^{a}\left[\frac{K(\rho)}{2}\left(\frac{d \Phi_{P}}{d \rho}\right)^{2}+\frac{L(\rho)}{2} \frac{d I^{2}}{d \rho}+V(\rho) \frac{d p}{d \rho}\right] d \rho
\end{aligned}
$$

subject to the constraints at the fixed boundary $\rho=a$, e.g., pressure $p(a)=0$ and total toroidal current $I_{T}(a)=$ constant. In other words, the equilibrium state (an excited state) corresponds to a balance between minimum internally stored magnetic energy in the plasma loop and maximum delivery of work of the plasma solenoid with $\delta J(a)=0$ for a fixed boundary. Integration by parts of the last two terms in $J(a)$ yields

$$
J(a)=\int_{0}^{a}\left[\frac{K(\rho)}{2}\left(\frac{d \Phi_{P}}{d \rho}\right)^{2}-\frac{I^{2}(\rho)-I^{2}(a)}{2} \frac{d L}{d \rho}-p(\rho) \frac{d V}{d \rho}\right] d \rho .
$$


According to the Lagrangian method, the functional $J(a)$ is written in the form

$$
J(a)=\int_{0}^{a} \mathcal{L}\left(\Phi_{P}, \Phi_{P}^{\prime}, \rho\right) d \rho
$$

with the Lagrangian density

$$
\begin{aligned}
\mathcal{L}\left(\Phi_{P}, \Phi_{P}^{\prime}, \rho\right)= & \frac{K(\rho)}{2}\left(\frac{d \Phi_{P}}{d \rho}\right)^{2}-\frac{I^{2}\left(\Phi_{P}\right)-I^{2}(a)}{2} \frac{d L}{d \rho} \\
& -p\left(\Phi_{P}\right) \frac{d V}{d \rho}
\end{aligned}
$$

and $\Phi_{P}^{\prime}=d \Phi_{P} / d \rho$. The Euler equation for a fixed boundary is

$$
\frac{\partial \mathcal{L}}{\partial \Phi_{P}}-\frac{d}{d \rho}\left(\frac{\partial \mathcal{L}}{\partial \Phi_{P}^{\prime}}\right)=0
$$

which is simply the flux-surface averaged Grad-Shafranov equation:

$$
\frac{d}{d \rho}\left(K(\rho) \frac{d \Phi_{P}}{d \rho}\right)+\frac{d V}{d \rho} \frac{d p}{d \Phi_{P}}+\frac{1}{2} \frac{d L}{d \rho} \frac{d I^{2}}{d \Phi_{P}}=0 .
$$

Hence, the functional $J(a)$ gives a variational principle for fixed-boundary equilibrium solutions in the form $\delta[J(a)]_{\Phi_{P}}$ $=0$. The poloidal flux function $\Phi_{P}$ is the generalized coordinate and $\Phi_{P}^{\prime}$ is the generalized velocity, with $\partial \mathcal{L} / \partial \Phi_{P}^{\prime}$ $=K(\rho) d \Phi_{P} / d \rho=I_{T}(\rho)$ corresponding to the generalized momentum. Consequently, the second-order Grad-Shafranov equation can be written as a system of two first-order canonical Euler equations

$$
\begin{aligned}
\frac{d \Phi_{P}}{d \rho} & =\frac{I_{T}(\rho)}{K(\rho)}, \\
\frac{d I_{T}}{d \rho} & =-\frac{d L}{d \rho} I(\rho) \frac{d I}{d \Phi_{P}}-\frac{d V}{d \rho} \frac{d p}{d \Phi_{P}} .
\end{aligned}
$$

Early formulations of magnetohydrodynamic equilibria in Lagrangian form have been carried out by Grad and Rubin ${ }^{4}$ and later extended by Grad. ${ }^{23}$ The derivation of the twodimensional Grad-Shafranov equation from a variational principle has been accomplished by Khait. ${ }^{24}$

Now consider the shape and the position of the plasma cross section characterized by a set of admissible functions $u_{i}(\rho)$, that is, sufficiently smooth functions that satisfy the boundary conditions. In the present article, each one of the Fourier amplitudes corresponds to such a function $u_{i}(\rho)$. Taking

$$
\begin{aligned}
& R=R\left(\rho, \theta, u_{i}\right), \\
& Z=Z\left(\rho, \theta, u_{i}\right),
\end{aligned}
$$

yields the scale factors and the Jacobian

$$
\begin{aligned}
h_{\theta} & =h_{\theta}\left(\rho, \theta, u_{i}\right), \\
h_{\zeta} & =h_{\zeta}\left(\rho, \theta, u_{i}\right), \\
\sqrt{g} & =\sqrt{g}\left(\rho, \theta, u_{i}, u_{i}^{\prime}\right),
\end{aligned}
$$

and the equilibrium coefficient derivatives

$$
\begin{aligned}
\frac{d V}{d \rho} & =\frac{\partial V}{\partial \rho}+\sum_{i} \frac{d u_{i}}{d \rho} \frac{\partial V}{\partial u_{i}}, \\
\frac{d L}{d \rho} & =\frac{\partial L}{\partial \rho}+\sum_{i} \frac{d u_{i}}{d \rho} \frac{\partial L}{\partial u_{i}}, \\
\frac{d K}{d \rho} & =\frac{\partial K}{\partial \rho}+\sum_{i} \frac{d u_{i}}{d \rho} \frac{\partial K}{\partial u_{i}}+\sum_{i} \frac{d u_{i}^{\prime}}{d \rho} \frac{\partial K}{\partial u_{i}^{\prime}},
\end{aligned}
$$

where $u_{i}^{\prime}=d u_{i} / d \rho$. For given profiles $I(\rho)$ and $p(\rho)$, one can find an extremum of the functional $J(a)$ defined on the space of the admissible functions $u_{i}(\rho)$. With the functional in the form

$$
J(a)=\int_{0}^{a}\left[\frac{K(\rho)}{2}\left(\frac{d \Phi_{P}}{d \rho}\right)^{2}+\frac{L(\rho)}{2} \frac{d I^{2}}{d \rho}+V(\rho) \frac{d p}{d \rho}\right] d \rho
$$

the related Euler equation for $u_{i}(\rho)$ is

$$
\begin{aligned}
& \frac{d}{d \rho}\left[\frac{1}{2} \frac{\partial K}{\partial u_{i}^{\prime}}\left(\frac{d \Phi_{P}}{d \rho}\right)^{2}\right]-\frac{1}{2} \frac{\partial K}{\partial u_{i}}\left(\frac{d \Phi_{P}}{d \rho}\right)^{2}-\frac{\partial V}{\partial u_{i}} \frac{d p}{d \rho}-\frac{1}{2} \frac{\partial L}{\partial u_{i}} \frac{d I^{2}}{d \rho} \\
& \quad=0
\end{aligned}
$$

subject to the fixed-boundary condition $\delta u_{i}(a)=0$. In terms of $I_{T}(\rho)$ and $p(\rho)$, the system of ordinary differential equations for the set of functions $u_{i}(\rho)$ is

$$
\frac{d}{d \rho}\left(\frac{I_{T}^{2}(\rho)}{2 K^{2}(\rho)} \frac{\partial K}{\partial u_{i}^{\prime}}\right)-\frac{I_{T}^{2}(\rho)}{2 K^{2}(\rho)} \frac{\partial K}{\partial u_{i}}-\frac{\partial V}{\partial u_{i}} \frac{d p}{d \rho}-\frac{1}{2} \frac{\partial L}{\partial u_{i}} \frac{d I^{2}}{d \rho}=0
$$

where

$$
\frac{d I^{2}}{d \rho}=-\frac{2}{d L / d \rho}\left(\frac{I_{T}(\rho)}{K(\rho)} \frac{d I_{T}}{d \rho}+\frac{d V}{d \rho} \frac{d p}{d \rho}\right)
$$

Considering the set $u_{i}(\rho)=\left\{R_{0}(\rho), Z_{0}(\rho), e(\rho), S_{2}(\rho)\right.$, $\left.A_{2}(\rho), S_{3}(\rho), A_{3}(\rho) \ldots\right\}$ of coefficients of the inverse mapping presented in Sec. II, the Euler equations for the Fourier amplitudes are equivalent to the variational moment equations. ${ }^{18}$

\section{APPROXIMATE SOLUTION OF THE EQUILIBRIUM EQUATIONS}

As discussed in Sec. IV, the Fourier coefficients $u_{i}(\rho)$ in the spectral representation of the flux surfaces satisfy the set of Euler equations

$$
\begin{aligned}
& \frac{d}{d \rho}\left(\frac{I_{T}^{2}(\rho)}{2 K^{2}(\rho)} \frac{\partial K}{\partial u_{i}^{\prime}}\right)-\frac{I_{T}^{2}(\rho)}{2 K^{2}(\rho)} \frac{\partial K}{\partial u_{i}}-\frac{\partial V}{\partial u_{i}} \frac{d p}{d \rho}+\frac{\partial L / \partial u_{i}}{d L / d \rho} \\
& \quad \times\left(\frac{I_{T}(\rho)}{K(\rho)} \frac{d I_{T}}{d \rho}+\frac{d V}{d \rho} \frac{d p}{d \rho}\right)=0 .
\end{aligned}
$$

These equations can be solved numerically for given profiles of the pressure, $p(\rho)$, and toroidal current, $I_{T}(\rho)$. 
Since $\rho=0$ is a critical point, the integration starts at a sufficiently small value $0 \lesssim \delta \rho \ll a$ with initial values guessed from the boundary conditions at the plasma edge $\rho=a$ as indicated by the Taylor series expansions:

$$
\begin{aligned}
& R_{0}(\delta \rho) \cong R_{m}-\left(R_{m}-R_{0}(a)\right) \frac{\delta \rho^{2}}{a^{2}} \\
& R_{0}^{\prime}(\delta \rho) \cong-2\left(R_{m}-R_{0}(a)\right) \frac{\delta \rho}{a^{2}}, \\
& Z_{0}(\delta \rho) \cong Z_{m}+\left(Z_{0}(a)-Z_{m}\right) \frac{\delta \rho^{2}}{a^{2}} \\
& Z_{0}^{\prime}(\delta \rho) \cong 2\left(Z_{0}(a)-Z_{m}\right) \frac{\delta \rho}{a^{2}}, \\
& e(\delta \rho) \cong \kappa_{m}+\left(e(a)-\kappa_{m}\right) \frac{\delta \rho^{2}}{a^{2}}, \\
& e^{\prime}(\delta \rho) \cong 2\left(e(a)-\kappa_{m}\right) \frac{\delta \rho}{a^{2}}, \\
& \cdots \\
& S_{n}(\delta \rho) \cong d S_{n} \delta \rho, \quad S_{n}^{\prime}(\delta \rho) \cong d S_{n}, \\
& A_{n}(\delta \rho) \cong d A_{n} \delta \rho, \quad A_{n}^{\prime}(\delta \rho) \cong d A_{n} .
\end{aligned}
$$

The parameters $R_{m}, Z_{m}, \kappa_{m}, \ldots d S_{n}$, and $d A_{n}$ are adjusted by iteration until the end-point conditions $R_{0}(a), Z_{0}(a), e(a), \ldots$ $S_{n}(a)$ and $A_{n}(a)$ are attained with sufficient precision (for a sufficiently small value of $\delta \rho$ the final adjusted parameters are independent of it). In this way, the set of coefficients $\left\{R_{0}(\rho), Z_{0}(\rho), e(\rho), \ldots S_{n}(\rho), A_{n}(\rho)\right\}$ can be precisely obtained for given $p(\rho)$ and $I_{T}(\rho)$. But the numerical procedure will not be pursued in this article.

An approximation to $R_{m}, Z_{m}, \kappa_{m}, R_{0}^{\prime \prime}(0), Z_{0}^{\prime \prime}(0), e^{\prime \prime}(0)$, $S_{2}^{\prime}(0), A_{2}^{\prime}(0), S_{3}^{\prime}(0)$, and $A_{3}^{\prime}(0)$ can be determined making power series in $\rho$ of both the flux function and the flux surface geometry, as discussed in Sec. III. The power series expansions of the Fourier coefficients, and of the geometric and magnetic coefficients in the equilibrium equation, are given in Appendix B. For simplicity, the presentation of these expansions is limited to the fourth-order, although sixth-order terms are needed in the final results. The expansions displayed in Appendix B are used only to obtain the terms in the expansion of each Euler equation as a power series in $\rho$. These terms are listed in Appendix $\mathrm{C}$ for increasing powers of $\rho$. The exponent of each term in the expansions increases by a factor of two with respect to the previous one. Cancelation of these terms relates the amplitudes of the Fourier coefficients around the magnetic axis. After the parameters $R_{m}, Z_{m}, \kappa_{m}, R_{0}^{\prime \prime}(0), Z^{\prime \prime}(0), e^{\prime \prime}(0)$, $S_{2}^{\prime}(0), A_{2}^{\prime}(0), S_{3}^{\prime}(0)$, and $A_{3}^{\prime}(0)$ have been calculated from the expanded Euler equations, as explained in the remainder of this section, and the (approximate) flux surface geometry has been fully determined, the equilibrium quantities can be evaluated using the expressions listed in Sec. IV.

The lowest-order terms in the expansions of the Euler equations for $R_{0}(\rho)$ and $Z_{0}(\rho)$ are given by Eqs. (C1) and $(\mathrm{C} 2)$, respectively. Information about the radial and vertical positions of the magnetic axis is contained in these equations that can be written as

$$
\begin{aligned}
& \left(3 \kappa_{m}^{2}+1\right) R_{m} R_{0}^{\prime \prime}(0)-8 R_{m} S_{2}^{\prime}(0) \\
& \quad+\kappa_{m}^{2}\left(1-\frac{8 \pi^{2}\left(\kappa_{m}^{2}+1\right)^{2} p^{\prime \prime}(0)}{\kappa_{m}^{2} \mu_{0} I_{T}^{\prime \prime}(0)^{2}}\right)=0
\end{aligned}
$$

and

$$
\left(\kappa_{m}^{2}+3\right) \frac{R_{m} Z_{0}^{\prime \prime}(0)}{\kappa_{m}}+4\left(\kappa_{m}^{2}-3\right) R_{m} A_{2}^{\prime}(0)=0,
$$

coupled to the equation for the magnetic axis elongation (C3) obtained from the lowest-order term of the Euler equation for $e(\rho)$

$$
\begin{aligned}
& 3\left(1+\frac{1}{\kappa_{m}^{2}}+\frac{4}{\kappa_{m}^{2}+1}\right)-3\left(3+\frac{3}{\kappa_{m}^{2}}-\frac{4}{\kappa_{m}^{2}+1}\right) R_{m}^{2} R_{0}^{\prime \prime}(0)^{2}-3\left(5+\frac{21}{\kappa_{m}^{2}}+\frac{4}{\kappa_{m}^{2}+1}\right) \frac{R_{m}^{2} Z_{0}^{\prime \prime}(0)^{2}}{\kappa_{m}^{2}} \\
& +3\left[3+\frac{1}{\kappa_{m}^{2}}+\frac{4}{\kappa_{m}^{2}+1}+16\left(3+\frac{3}{\kappa_{m}^{2}}+\frac{2}{\kappa_{m}^{2}+1}\right) R_{m} S_{2}^{\prime}(0)\right] R_{m} R_{0}^{\prime \prime}(0) \\
& +48\left(\frac{9}{\kappa_{m}^{2}}+\frac{4}{\kappa_{m}^{2}+1}\right) R_{m} A_{2}^{\prime}(0) \frac{R_{m} Z_{0}^{\prime \prime}(0)}{\kappa_{m}}+3\left(1+\frac{5}{\kappa_{m}^{2}}+\frac{12}{\kappa_{m}^{2}+1}\right) \frac{R_{m}^{2} e^{\prime \prime}(0)}{\kappa_{m}} \\
& +12\left[3-\frac{5}{\kappa_{m}^{2}}+\frac{4}{\kappa_{m}^{2}+1}-\left(19+\frac{23}{\kappa_{m}^{2}}+\frac{20}{\kappa_{m}^{2}+1}\right) R_{m} S_{2}^{\prime}(0)\right] R_{m} S_{2}^{\prime}(0) \\
& -12\left(5+\frac{41}{\kappa_{m}^{2}}+\frac{28}{\kappa_{m}^{2}+1}\right) R_{m}^{2} A_{2}^{\prime}(0)^{2}-3\left(1-\frac{1}{\kappa_{m}^{2}}\right) \frac{40 R_{m}^{2} S_{3}^{\prime}(0)}{a} \\
& +\left(1-\frac{1}{\kappa_{m}^{2}}\right)\left(\frac{R_{m}^{2} I_{T}^{(4)}(0)}{I_{T}^{\prime \prime}(0)}\right)-3\left[1-4 R_{m} R_{0}^{\prime \prime}(0)+8 R_{m} S_{2}^{\prime}(0)\right]\left(\frac{8 \pi^{2}\left(\kappa_{m}^{2}+1\right)^{2} p^{\prime \prime}(0)}{\kappa_{m}^{2} \mu_{0} I_{T}^{\prime \prime}(0)^{2}}\right)=0 .
\end{aligned}
$$

Note that these relationships involving derivatives on the magnetic axis are exact, no matter what the order of the power series approximation in $\rho$. Near the magnetic axis, the Fourier amplitudes are given by (the antisymmetric 
quadrangular coefficient $A_{3}(\rho)$ does not contribute near the axis or to the equilibrium coefficients within the fourth-order in $\rho$ )

$$
\begin{aligned}
R_{0}(\rho) & =R_{m}+\frac{\rho^{2}}{2} R_{0}^{\prime \prime}(0)+\cdots, \\
Z_{0}(\rho) & =Z_{m}+\frac{\rho^{2}}{2} Z_{0}^{\prime \prime}(0)+\cdots, \\
e(\rho) & =\kappa_{m}+\frac{\rho^{2}}{2} e_{0}^{\prime \prime}(0)+\cdots, \\
S_{2}(\rho) & =\rho S_{2}^{\prime}(0)+\cdots, \\
A_{2}(\rho) & =\rho A_{2}^{\prime}(0)+\cdots, \\
S_{3}(\rho) & =\rho S_{3}^{\prime}(0)+\cdots .
\end{aligned}
$$

Now the simplest approximation is obtained truncating the above power series. In this case, the parameters $R_{0}^{\prime \prime}(0)$, $Z_{0}^{\prime \prime}(0), e^{\prime \prime}(0), S_{2}^{\prime}(0), A_{2}^{\prime}(0)$, and $S_{3}^{\prime}(0)$ are determined by the plasma shape as

$$
\begin{aligned}
& R_{0}^{\prime \prime}(0) \cong-\frac{2\left(R_{m}-R_{0}(a)\right)}{a^{2}}, \quad Z_{0}^{\prime \prime}(0) \cong \frac{2\left(Z_{0}(a)-Z_{m}\right)}{a^{2}} \\
& e^{\prime \prime}(0) \cong \frac{2\left(e(a)-\kappa_{m}\right)}{a^{2}}, \quad S_{2}^{\prime}(0) \cong \frac{S_{2}(a)}{a}, \\
& A_{2}^{\prime}(0) \cong \frac{A_{2}(a)}{a}, \quad S_{3}^{\prime}(0) \cong \frac{S_{3}(a)}{a}
\end{aligned}
$$

so that

$$
\begin{aligned}
& R_{0}(\rho) \cong R_{m}-\left(R_{m}-R_{0}(a)\right) \frac{\rho^{2}}{a^{2}} \\
& Z_{0}(\rho) \cong Z_{m}+\left(Z_{0}(a)-Z_{m}\right) \frac{\rho^{2}}{a^{2}} \\
& e(\rho) \cong \kappa_{m}+\left(e(a)-\kappa_{m}\right) \frac{\rho^{2}}{a^{2}}, \quad S_{2}(\rho) \cong S_{2}(a) \frac{\rho}{a}, \\
& A_{2}(\rho) \cong A_{2}(a) \frac{\rho}{a}, \quad S_{3}(\rho) \cong S_{3}(a) \frac{\rho}{a} .
\end{aligned}
$$

Using these approximations, Eqs. (76) and (77) for the magnetic axis position give

$$
\begin{aligned}
R_{m} \cong & \frac{R_{0}(a)}{2}-\frac{2 a S_{2}(a)}{3 \kappa_{m}^{2}+1}+\left[\left(\frac{R_{0}(a)}{2}-\frac{2 a S_{2}(a)}{3 \kappa_{m}^{2}+1}\right)^{2}\right. \\
& \left.+\frac{\kappa_{m}^{2} a^{2}}{2\left(3 \kappa_{m}^{2}+1\right)}\left(1-\frac{\left(\kappa_{m}^{2}+1\right)^{2}}{\kappa_{m}^{2}} \frac{8 \pi^{2} p^{\prime \prime}(0)}{\mu_{0} I_{T}^{\prime \prime}(0)^{2}}\right)\right]^{1 / 2} \\
Z_{m} \cong & Z_{0}(a)-\frac{2 \kappa_{m}\left(3-\kappa_{m}^{2}\right)}{3+\kappa_{m}^{2}} a A_{2}(a) .
\end{aligned}
$$

Note that only the difference $Z_{0}(a)-Z_{m}$ is relevant for the internal equilibrium of the plasma, as expected. The elongation $\kappa_{m}$ is calculated from Eq. (78), which is denoted by $f\left(\kappa_{m}\right)=0 ; \kappa_{m}$ can be determined numerically using the Newton-Raphson formula

$$
\kappa_{i+1}=\kappa_{i}-\frac{f\left(\kappa_{i}\right)}{f^{\prime}\left(\kappa_{i}\right)} .
$$

The value of the elongation at the plasma boundary, $e(a)$, is taken as a trial solution, giving a very good approximation to the true root using the two or three step Newton-Raphson method. This crude approximation for the Fourier amplitudes and for the magnetic axis parameters is appropriate for largeaspect-ratio configurations and was used to represent the magnetic islands in the core of a tokamak. ${ }^{21}$

The next order approximation for the tokamak equilibrium can be obtained taking again into account the boundary conditions and writing the position of the major radius in the form

$$
R_{0}(\rho) \cong R_{m}+\frac{\rho^{2}}{2} R_{0}^{\prime \prime}(0)+\left(R_{0}(a)-R_{m}-\frac{1}{2} a^{2} R_{0}^{\prime \prime}(0)\right) \frac{\rho^{4}}{a^{4}}
$$

with similar expressions for $Z_{0}(\rho)$ and $e(\rho)$. The symmetric triangularity coefficient is approximated by

$$
S_{2}(\rho) \cong \rho S_{2}^{\prime}(0)+\left(S_{2}(a)-a S_{2}^{\prime}(0)\right) \frac{\rho^{3}}{a^{3}},
$$

with similar expressions for $A_{2}(\rho), S_{3}(\rho)$ and $A_{3}(\rho)$. The coefficients $R_{m}, Z_{m}, \kappa_{m}, R_{0}^{\prime \prime}(0), Z_{0}^{\prime \prime}(0), e^{\prime \prime}(0), S_{2}^{\prime}(0), A_{2}^{\prime}(0)$, $S_{3}^{\prime}(0)$, and $A_{3}^{\prime}(0)$ are determined using Eqs. (76)-(78) coupled with Eqs. (C8)-(C14). In this way, the series truncation is moved nearer to the plasma border, generating simple, fast equilibrium solutions. However, improved solutions can be obtained introducing sectionally continuous approximations for the Fourier coefficients, as described in the following.

The sectionally continuous approximations are constructed by matching the Taylor series in the plasma core with the series in the plasma border, as illustrated for the position of the major radius $R_{0}(\rho)$ :

$$
\begin{aligned}
& R_{0, \text { core }}(\rho)=R_{m}+\frac{\rho^{2}}{2} R_{0}^{\prime \prime}(0)+\frac{\rho^{4}}{24} R_{0}^{(4)}(0)+\cdots \quad 0 \leqslant \rho \leqslant \rho_{*}, \\
& R_{0, \text { edge }}(\rho)=R_{0}(a)-(a-\rho) R_{0}^{\prime}(a)+\frac{(a-\rho)^{2}}{2} R_{0}^{\prime \prime}(a)+\cdots \\
& \quad \rho * \leqslant \rho \leqslant a .
\end{aligned}
$$

Defining a matching radius $\rho_{*}$ such that

$$
\begin{aligned}
& R_{0, \text { core }}\left(\rho_{*}\right)=R_{0, \text { edge }}\left(\rho_{*}\right), \quad R_{0, \text { core }}^{\prime}\left(\rho_{*}\right)=R_{0, \text { edge }}^{\prime}\left(\rho_{*}\right), \\
& \quad \text { and } \quad R_{0, \text { core }}^{\prime \prime}\left(\rho_{*}\right)=R_{0, \text { edge }}^{\prime \prime}\left(\rho_{*}\right),
\end{aligned}
$$

one obtains

$$
\begin{aligned}
& R_{0}^{\prime \prime}(0) \\
& =\frac{-4\left(3 a-2 \rho_{*}\right)\left(R_{m}-R_{0}(a)\right)-\left(6 a^{2}-8 a \rho_{*}+3 \rho_{*}^{2}\right) R_{0}^{\prime}(a)}{a\left(4 a-3 \rho_{*}\right) \rho_{*}} \\
& R_{0}^{(4)}(0)=\frac{12\left(2\left(R_{m}-R_{0}(a)\right)+a R_{0}^{\prime}(a)\right)}{\left(4 a-3 \rho_{*}\right) \rho_{*}^{3}}, \\
& R_{0}^{\prime \prime}(a)=\frac{8\left(R_{m}-R_{0}(a)\right)+\left(8 a-3 \rho_{*}\right) R_{0}^{\prime}(a)}{a\left(4 a-3 \rho_{*}\right)}
\end{aligned}
$$

with similar expressions for $Z_{0}(\rho)$ and $e(\rho)$. Likewise, the sectionally continuous approximation for the symmetric triangularity coefficient is: 


$$
\begin{aligned}
S_{2, \text { core }}(\rho)= & \rho S_{2}^{\prime}(0)+\frac{\rho^{3}}{6} S_{2}^{(3)}(0)+\cdots \quad 0 \leqslant \rho \leqslant \rho_{*}, \\
S_{2, \text { edge }}(\rho)= & S_{2}(a)-(a-\rho) S_{2}^{\prime}(a) \\
& +\frac{(a-\rho)^{2}}{2} S_{2}^{\prime \prime}(a)+\cdots \quad \rho_{*} \leqslant \rho \leqslant a .
\end{aligned}
$$

Using the same matching radius $\rho_{*}$ such that

$S_{2, \text { core }}\left(\rho_{*}\right)=S_{2, \text { edge }}\left(\rho_{*}\right), \quad S_{2, \text { core }}^{\prime}\left(\rho_{*}\right)=S_{2, \text { edge }}^{\prime}\left(\rho_{*}\right), \quad$ and

$S_{2, \text { core }}^{\prime \prime}\left(\rho_{*}\right)=S_{2, \text { edge }}^{\prime \prime}\left(\rho_{*}\right)$,

one obtains

$$
\begin{aligned}
S_{2}^{\prime}(0) & =\frac{3\left(2 a-\rho_{*}\right) S_{2}(a)-\left(3 a^{2}-3 a \rho_{*}+\rho_{*}^{2}\right) S_{2}^{\prime}(a)}{3 a^{2}-\rho_{*}^{2}}, \\
S_{2}^{(3)}(0) & =-\frac{6\left(S_{2}(a)-a S_{2}^{\prime}(a)\right)}{\left(3 a^{2}-\rho_{*}^{2}\right) \rho_{*}} \\
S_{2}^{\prime \prime}(a) & =-\frac{6\left(S_{2}(a)-a S_{2}^{\prime}(a)\right)}{3 a^{2}-\rho_{*}^{2}}
\end{aligned}
$$

with similar expressions for $A_{2}(\rho), S_{3}(\rho)$ and $A_{3}(\rho)$.

In this way, the flux surface geometry is defined in terms of the magnetic axis parameters, $R_{m}, Z_{m}$, and $\kappa_{m}$, the shape of the plasma, $a, R_{0}(a), Z_{0}(a), e(a), S_{2}(a), A_{2}(a), S_{3}(a)$, $A_{3}(a)$ (Dirichlet parameters), the radial derivatives at the plasma boundary, $R_{0}^{\prime}(a), Z_{0}^{\prime}(a), e^{\prime}(a), S_{2}^{\prime}(a), A_{2}^{\prime}(a), S_{3}^{\prime}(a)$, $A_{3}^{\prime}(a)$ (Neumann parameters), and the matching radius, $\rho_{*}$. Now the magnetic axis and the set of Neumann parameters are determined for given Dirichlet conditions (plasma shape) by solving Eqs. (76)-(78) coupled with Eqs. (C8)-(C14). There are ten equations available for ten variables, with $\rho_{*}$ as an additional variable. The optimum matching radius $\rho_{*}$ is determined by a direct variational procedure searching for a stationary value of the internal energy $U(a)$ given in Sec. IV by Eq. (58). Conversely, the magnetic axis parameters, $R_{m}$, $Z_{m}$, and $\kappa_{m}$, the Dirichlet parameters, $a, e(a), S_{2}(a)$, and $A_{2}(a), S_{3}(a), A_{3}(a)$, and the matching radius $\rho_{*}$ can be determined for given Neumann conditions, $R_{0}^{\prime}(a), Z_{0}^{\prime}(a)$, $e^{\prime}(a), S_{2}^{\prime}(a), A_{2}^{\prime}(a), S_{3}^{\prime}(a)$, and $A_{3}^{\prime}(a)$, by solving the same Eqs. (76)-(78) coupled with Eqs. (C8)-(C14). Again, there are ten equations for ten variables, including $\rho_{*}$. Since the Neumann conditions are gradients, it is necessary to specify a fixed point in order to obtain a unique answer. A convenient choice is to specify the position of the major axis through the pair of coordinates $R_{0}(a)$ and $Z_{0}(a)$. The same solution is obtained using either Dirichlet or Neumann boundary conditions, interchangeably.

Note that a simpler sectionally continuous approximation can be constructed by reducing the order of the Taylor series in the plasma core and calculating the magnetic axis parameters, $R_{m}, Z_{m}$, and $\kappa_{m}$ from the given Dirichlet parameters by means of Eqs. (76)-(78) only. The matching radius is determined, as before, searching for a stationary state of the internal energy $U(a)$. Such approximation should be useful for fast simulations of the equilibrium evolution. An extended sectionally continuous approximation is used in solving the free-boundary problem presented in the Paper II. ${ }^{1}$

\section{APPLICATION TO AN ITER-LIKE TOKAMAK}

The approximate solution developed in Sec. V using the sectionally continuous approximations (86) and (89) for the Fourier amplitudes is applied to an ITER-like tokamak. The main geometric parameters of the ITER tokamak are $R=6.2 \mathrm{~m}, Z=0.55 \mathrm{~m}$, and $a=2.0 \mathrm{~m} .^{25}$ A slightly up-down asymmetric configuration is assumed with the upper elongation and triangularity given by $\kappa_{1}=1.8$ and $\delta_{1}=0.40$, and the lower elongation and triangularity given by $\kappa_{2}=1.8$ and $\delta_{2}=0.45$, in respective order. Figure 1 shows a cross section of the ITER-like tokamak indicating the central and extreme points of the plasma boundary. As discussed in Sec. II, the vertical position $Z$ and the fixed points corresponding to the values $R+a, R-a, Z+\kappa_{1} a, Z-\kappa_{2} a, R-\delta_{1} a$, and $R-\delta_{2} a$ can be adjusted with the set of Fourier amplitudes $R_{0}(a)$, $Z_{0}(a), e(a), S_{2}(a), A_{2}(a), S_{3}(a)$, and $A_{3}(a)$ on the plasma boundary $\rho=a$ (Dirichlet parameters) listed in Table I.

In order to solve the Euler equations, or the GradShafranov equation for that matter, it is necessary to specify the profiles of the plasma pressure $p(\rho)$ and of the toroidal current $I_{T}(\rho)$. The power series expansions for the Euler equations presented in Appendix $\mathrm{C}$ depend on $p^{\prime \prime}(0)$, $p^{(4)}(0), I_{T}^{\prime \prime}(0), I_{T}^{(4)}(0)$, and $I_{T}^{(6)}(0)$ as free parameters. In this article, the pressure is represented by an eight-order polynomial in $\rho$

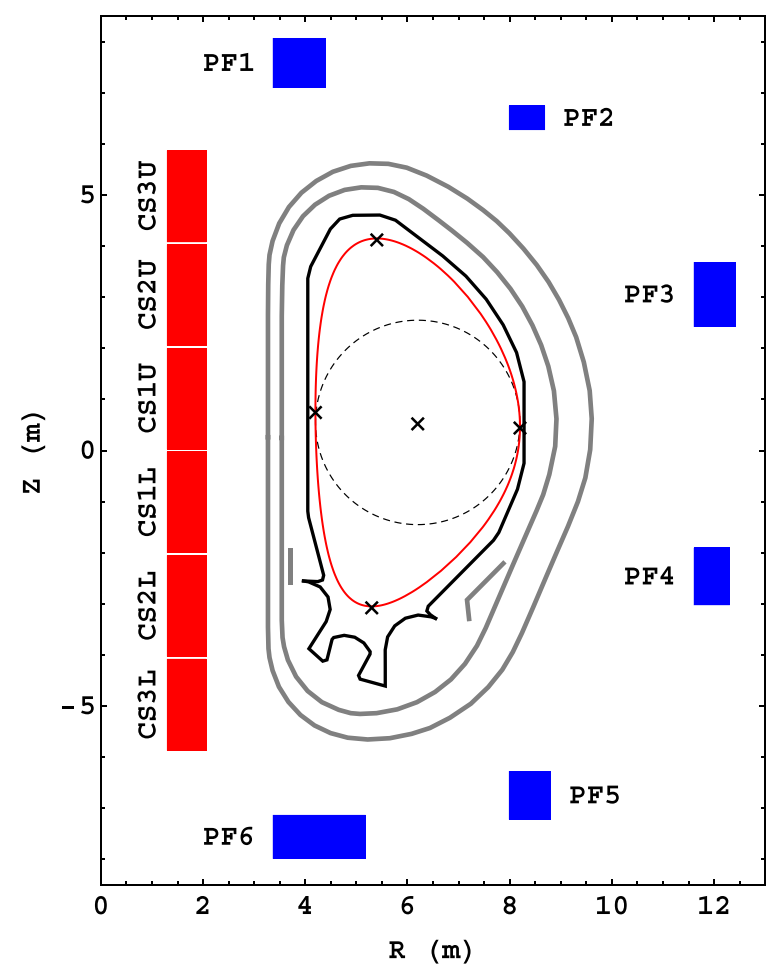

FIG. 1. ITER-like cross section: the crosses indicate the central point $(R=6.2 \mathrm{~m}, Z=0.55 \mathrm{~m})$ and the extreme points $\left(R_{1}, Z_{1}\right),\left(R_{2}, Z_{2}\right),\left(R_{3}, Z_{3}\right)$, and $\left(R_{4}, Z_{4}\right)$ of the plasma boundary; the dotted circle corresponds to the minor radius $a=2.0 \mathrm{~m}$; the plasma is surrounded by the limiter and vacuum vessel walls; the rectangles represent the poloidal cross sections of the central solenoid (CS) and equilibrium field coils (PF). 
TABLE I. Geometric parameters which describe the plasma boundary of the ITER-like tokamak shown in Fig. 1.

\begin{tabular}{lcc}
\hline \hline Parameter set & \multicolumn{2}{c}{ Values } \\
\hline Dirichlet & $R_{0}(a)=6.2 \mathrm{~m}$ & $Z_{0}(a)=0.55 \mathrm{~m}$ \\
& $a=2.0 \mathrm{~m}$ & $e(a)=1.75718$ \\
& $S_{2}(a)=0.109542$ & $A_{2}(a)=-0.00344217$ \\
Extreme points & $S_{3}(a)=-0.00734624$ & $A_{3}(a)=-0.00817006$ \\
& $R_{1}(a)=5.400 \mathrm{~m}$ & $Z_{1}(a)=4.150 \mathrm{~m}$ \\
& $R_{2}(a)=5.300 \mathrm{~m}$ & $Z_{2}(a)=-3.050 \mathrm{~m}$ \\
$R_{3}(a)=8.201 \mathrm{~m}$ & $Z_{3}(a)=0.469 \mathrm{~m}$ \\
& $R_{4}(a)=4.199 \mathrm{~m}$ & $Z_{4}(a)=0.772 \mathrm{~m}$ \\
\hline \hline
\end{tabular}

$$
\begin{aligned}
p(\rho)= & p_{0}+\frac{\rho^{2}}{2} p^{\prime \prime}(0)-\frac{\rho^{4}}{8 a^{4}}\left(48 p_{0}+12 a^{2} p^{\prime \prime}(0)-a^{2} p^{\prime \prime}(a)\right) \\
& +\frac{\rho^{6}}{4 a^{6}}\left(32 p_{0}+6 a^{2} p^{\prime \prime}(0)-a^{2} p^{\prime \prime}(a)\right) \\
& -\frac{\rho^{8}}{8 a^{8}}\left(24 p_{0}+4 a^{2} p^{\prime \prime}(0)-a^{2} p^{\prime \prime}(a)\right),
\end{aligned}
$$

which satisfies the constraints $p(a)=0$ and $p^{\prime}(a)=0$, along with $p_{0}>0$ and $p^{\prime}(0)=0$. With these constraints, the free parameter $p^{(4)}(0)$ is replaced by $p^{\prime \prime}(a)$, besides the fundamental quantity $p(0)=p_{0}$ and the remaining free parameter $p^{\prime \prime}(0)$. Furthermore, $p^{\prime \prime}(0)$ is replaced by $-2 \alpha_{p} p_{0} / a^{2}$ such that near the magnetic axis the pressure profile approaches the standard binomial form

$$
p(\rho) \sim p_{0}\left(1-\frac{\rho^{2}}{a^{2}}\right)^{\alpha_{p}}
$$

with a pressure peaking factor $\alpha_{p}$. The conditions for positive pressure in the range $0 \leqslant \rho \leqslant a$ are

$$
p^{\prime \prime}(a) \geqslant 0 \quad \text { and } \quad-\frac{a^{2} p^{\prime \prime}(0)}{2 p_{0}}=\alpha_{p} \leqslant 4+\sqrt{\frac{a^{2} p^{\prime \prime}(a)}{2 p_{0}}} .
$$

Peaked profiles correspond to $0<\alpha_{p} \leqslant 4$ (a hollow profile is obtained with $\alpha_{p}<0$ ) giving a pressure distribution described by three parameters $p_{0}, \alpha_{p}$, and $p^{\prime \prime}(a)$. The pressure on the magnetic axis is given by

$$
p_{0}=\left(10^{3} e\right) n_{e}(0)\left(T_{e}(0)+\frac{T_{i}(0)}{Z_{\mathrm{eff}}}\right),
$$

where $n_{e}(0)$ is the electron plasma density; $T_{e}(0)$ and $T_{i}(0)$ are the electron and ion temperatures in $\mathrm{keV}$, respectively;
$Z_{\text {eff }}$ is the effective charge number; and $e$ is the electron charge. Throughout this article, the plasma pressure on the axis is fixed with $n_{e}(0)=10^{20} \mathrm{~m}^{-3}, T_{e}(0)=T_{i}(0)=10$ $\mathrm{keV}$, and $Z_{\text {eff }}=3 / 2$.

The toroidal current profile can be represented by a tenth-order polynomial in $\rho$ which satisfies the constraint $I_{T}^{\prime}(a)=0$ with $I_{T}(0)=0, I_{T}^{\prime}(0)=0$ and $I_{T}(a)>0$ (the plasma current is assumed positive). The free parameters are $I_{T}^{\prime \prime}(0)>0, I_{T}^{(4)}(0)$ and $I_{T}^{(6)}(0)$. The parameter $I_{T}^{(6)}(0)$ can be substituted by $I_{T}^{\prime \prime}(a)<0$, besides $I_{T}(a)>0, I_{T}^{(4)}(0)$ and $I_{T}^{\prime \prime}(0)>0$. However, a set of four parameters results in nine conditions for positive toroidal current in the range $0 \leqslant \rho \leqslant a$. Therefore, in this paper the toroidal current profile is represented, as well as the pressure profile, by an eight-order polynomial in $\rho$

$$
\begin{aligned}
I_{T}(\rho)= & \frac{\rho^{2}}{2} I_{T}^{\prime \prime}(0)+\frac{\rho^{4}}{8 a^{4}}\left[48 I_{T}(a)-12 a^{2} I_{T}^{\prime \prime}(0)+a^{2} I_{T}^{\prime \prime}(a)\right] \\
& -\frac{\rho^{6}}{4 a^{6}}\left[32 I_{T}(a)-6 a^{2} I_{T}^{\prime \prime}(0)+a^{2} I_{T}^{\prime \prime}(a)\right] \\
& +\frac{\rho^{8}}{8 a^{8}}\left[24 I_{T}(a)-4 a^{2} I_{T}^{\prime \prime}(0)+a^{2} I_{T}^{\prime \prime}(a)\right],
\end{aligned}
$$

such that $I_{T}^{\prime}(a)=0$. Replacing $I_{T}^{\prime \prime}(0)$ by $2\left(1+\alpha_{j}\right) I_{T}(a) / a^{2}$ the toroidal current approaches, near the magnetic axis, the standard binomial profile

$$
I_{T}(\rho) \sim I_{T}(a)\left[1-\left(1-\frac{\rho^{2}}{a^{2}}\right)^{1+\alpha_{j}}\right]
$$

with a current peaking factor $\alpha_{j}$. In this way, the toroidal current distribution is described by a reduced set of three parameters $I_{T}(a), \alpha_{j}$, and $I_{T}^{\prime \prime}(a)$. The flux-averaged toroidal current density is approximately given by

$$
\left\langle j_{T}\right\rangle(\rho) \cong \frac{1}{2 \pi \kappa_{m} \rho} \frac{d I_{T}}{d \rho} .
$$

Note that this is a simple approximation, the correct distribution being obtained by taking the flux-surface average of $j_{T}(\rho, \theta)$ defined by Eq. (29). Nevertheless, on the magnetic axis one obtains exactly

$$
\left\langle j_{T}\right\rangle(\rho) \underset{\rho \rightarrow 0}{\rightarrow} \frac{I_{T}^{\prime \prime}(0)}{2 \pi \kappa_{m}}=\frac{\left(1+\alpha_{j}\right) I_{T}(a)}{\pi \kappa_{m} a^{2}}=j_{T}(0) .
$$

The conditions for positive toroidal current in the range $0 \leqslant \rho \leqslant a$ are

$$
\begin{cases}-\frac{48 I_{T}(a)}{a^{2}} \leqslant I_{T}^{\prime \prime}(a) \leqslant 0 & \text { and } I_{T}^{\prime \prime}(0) \geqslant 0 \\ & \text { or } \\ I_{T}^{\prime \prime}(a)<-\frac{48 I_{T}(a)}{a^{2}} & \text { and } I_{T}^{\prime \prime}(0) \geqslant \frac{I_{T}^{\prime \prime}(a)}{3}+\left[\frac{176}{27}+\frac{\sqrt{2}}{216}\left(-\frac{3 a^{2} I_{T}^{\prime \prime}(a)}{I_{T}(a)}-16\right)^{3 / 2}\right] \frac{I_{T}(a)}{a^{2}} .\end{cases}
$$


For simplicity, only the first condition is considered in this paper.

A Taylor expansion of the equilibrium equation (40) around the magnetic axis gives

$$
I(\rho)=I(0)+\frac{I^{\prime \prime}(0)}{2} \rho^{2}+\cdots
$$

where $I(0)=2 \pi R_{m} B_{m} / \mu_{0}$ and

$$
I^{\prime \prime}(0)=-\frac{2 \pi R_{m}}{B_{m}}\left(\frac{\mu_{0} \kappa_{m}^{2} j_{T}^{2}(0)}{\kappa_{m}^{2}+1}+p^{\prime \prime}(0)\right)<0
$$

for a standard monotonic poloidal current profile (without reversed shear and current holes). In terms of the pressure profile peaking factor, this gives

$$
\alpha_{p}<\frac{\mu_{0} \kappa_{m}^{2} a^{2} j_{T}^{2}(0)}{2\left(\kappa_{m}^{2}+1\right) p_{0}}
$$

Likewise, a Taylor expansion of the equilibrium equation (40) at the plasma boundary gives

$$
I(\rho)=I(a)+\frac{I^{\prime \prime}(a)}{2}(a-\rho)^{2}+\cdots
$$

where $I(a)=2 \pi R_{0}(a) B_{0} / \mu_{0}$ and

$$
I^{\prime \prime}(a)=-\frac{1}{I(a) L^{\prime}(a)}\left(\frac{I_{T}(a) I_{T}^{\prime \prime}(a)}{K(a)}+V^{\prime}(a) p^{\prime \prime}(a)\right)
$$

Since $V^{\prime}(a)>0, L^{\prime}(a)>0, K(a)>0, I(a)>0, I_{T}(a)$ $>0, \quad I_{T}^{\prime \prime}(a) \leqslant 0, p^{\prime \prime}(a) \geqslant 0$, and $I^{\prime \prime}(a) \geqslant 0$ for a standard monotonic poloidal current profile, it follows that

TABLE II. ITER-like tokamak equilibria obtained for various values of the peaking factors $\alpha_{n}$ and $\alpha_{T}$ of the density and temperature profiles, respectively. The plasma current in MA corresponds to the maximum value consistent with a central $q(0)$ value close to one, but limited by the nominal value $I_{T}(a)=15 \mathrm{MA}$. The table also gives the values of the matching radius $\rho_{*}$ in $\mathrm{m}$, the internal energy $U(a)$ in $\mathrm{MJ}$, the poloidal flux $\Phi_{P}(a)$ in $\mathrm{Wb}$, and the edge safety factor $q(a)$.

\begin{tabular}{lcccccccc}
\hline \hline Profiles & $\alpha_{\mathrm{n}}$ & $\alpha_{\mathrm{T}}$ & $I_{T}(a)$ & $\rho_{*}$ & $U(a)$ & $\Phi_{P}(a)$ & $q(0)$ & $q(a)$ \\
\hline Peaked & $2 \alpha_{T}$ & 1.4 & 7.22 & 1.356 & 206.5 & 49.45 & 1.000 & 7.171 \\
Peaked & $2 \alpha_{T}$ & 1.3 & 7.78 & 1.596 & 234.8 & 51.86 & 1.000 & 6.727 \\
Peaked & $2 \alpha_{T}$ & 1.2 & 8.50 & 1.972 & 274.1 & 55.01 & 1.000 & 6.107 \\
Intermediate & $3 \alpha_{T} / 2$ & 1.1 & 9.31 & 1.830 & 320.4 & 58.18 & 1.000 & 5.516 \\
Intermediate & $\alpha_{\mathrm{T}}$ & 1.0 & 10.33 & 1.585 & 383.6 & 62.16 & 1.000 & 4.832 \\
Intermediate & $\alpha_{\mathrm{T}}$ & 0.9 & 11.64 & 1.690 & 473.2 & 67.35 & 1.000 & 4.218 \\
Intermediate & $\alpha_{\mathrm{T}}$ & 0.8 & 13.51 & 1.258 & 615.6 & 74.49 & 1.000 & 3.498 \\
Flat & $\alpha_{T} / 2$ & 0.8 & 13.48 & 1.187 & 612.8 & 74.29 & 1.000 & 3.502 \\
Flat & $\alpha_{T} / 2$ & 0.739 & 15.00 & 0.825 & 741.4 & 79.99 & 1.000 & 3.104 \\
Flat & $\alpha_{T} / 2$ & 0.7 & 15.00 & 0.577 & 731.6 & 78.41 & 1.058 & 3.095 \\
\hline \hline
\end{tabular}

$$
0 \leqslant p^{\prime \prime}(a) \leqslant-\frac{I_{T}(a) I_{T}^{\prime \prime}(a)}{K(a) V^{\prime}(a)}
$$

Table II lists the results of equilibrium calculations performed for the ITER-like tokamak with varying peaking factors for the density, $\alpha_{n}$, and temperature, $\alpha_{T}$. The pressure peaking factor is given by $\alpha_{p}=\alpha_{n}+\alpha_{T}$, which defines the parameter $p^{\prime \prime}(0)=-2 \alpha_{p} p_{0} / a^{2}$. The current density peaking factor is given in terms of the temperature peaking factor by $\alpha_{j}=3 \alpha_{T} / 2$ for an ohmically relaxed state $\left(j \sim T^{3 / 2}\right)$, defining the parameter $I_{T}^{\prime \prime}(0)=2\left(1+\alpha_{j}\right) I_{T}(a) / a^{2}$. For simplicity, the remaining free parameters $p^{\prime \prime}(a)$ and $I_{T}^{\prime \prime}(a)$ are put equal to zero. The plasma pressure on the axis is fixed at $p_{0}=267 \mathrm{kPa}$ and the external toroidal magnetic field on the geometric center is $B_{0}=5.3 \mathrm{~T}$. The geometry of the plasma boundary is fixed as given in Table I and the plasma current listed in Table II reaches a value consistent with a safety factor $q(0)$ equal or close to one on the magnetic axis, but limited to the nominal value $I_{T}(a)=15 \mathrm{MA}$ expected for ITER. The equilibria are arbitrarily divided into three classes, ranging from peaked to flat profiles. As the current profile becomes flatter as given by $\alpha_{j}=3 \alpha_{T} / 2$, the pressure profile also flattens to avoid poloidal current density reversal according to the general conditions (103) and (106). The flat profiles with $\alpha_{n}=\alpha_{T} / 2$ give $\alpha_{p}=3 \alpha_{T} / 2=\alpha_{j}$, which corresponds to stationary magnetic entropy states $(j \sim p) .{ }^{26}$ In particular, the equilibrium with $\alpha_{T}=0.739$ gives a central $q(0)$ value close to one with the nominal plasma current $I_{T}(a)=15$ MA and an edge safety factor $q(a)$ just above three. This equilibrium gives nearly the maximum value of the internal energy $U(a)$ without increasing the plasma current above its nominal value.

Now Table III lists the magnetic axis, matching radius, and Neumann parameters for the peaked equilibrium corresponding to the first line in Table II. Figure 2 shows the flux surface geometry, the plasma pressure profile, $p(\rho)$, the toroidal current profile, $I_{T}(\rho)$, and the approximate toroidal current density profile, $\left\langle j_{T}\right\rangle(\rho)$ for this equilibrium. Furthermore, Fig. 3 shows the variation with the radial variable $\rho$ of the poloidal flux, $\Phi_{P}(\rho)$, of the main Fourier amplitudes, $R_{0}(\rho), Z_{0}(\rho)$ and $e(\rho)$, of the triangularity coefficients, $S_{2}(\rho)$ and $A_{2}(\rho)$, and of the quadrangularity coefficients, $S_{3}(\rho)$ and $A_{3}(\rho)$.

TABLE III. Magnetic axis, matching radius, and Neumann parameters for the ITER-like tokamak equilibrium with peaked pressure and current density profiles shown in Fig. 2, and discussed in the text.

\begin{tabular}{lcc}
\hline \hline Parameter set & Values & \\
\hline Magnetic axis & $R_{\mathrm{m}}=6.47327 \mathrm{~m}$ & $Z_{\mathrm{m}}=0.549442 \mathrm{~m}$ \\
& $\kappa_{m}=1.34389$ & \\
Matching radius & $\rho_{*}=1.35609 \mathrm{~m}$ & \\
Neumann & $R_{0}^{\prime}(a)=-0.479432$ & $Z_{0}^{\prime}(a)=0.0049004$ \\
& $e^{\prime}(a)=0.425214 \mathrm{~m}^{-1}$ & \\
& $S_{2}^{\prime}(a)=-0.00677738 \mathrm{~m}^{-1}$ & $A_{2}^{\prime}(a)=-0.000911109 \mathrm{~m}^{-1}$ \\
& $S_{3}^{\prime}(a)=0.00821685 \mathrm{~m}^{-1}$ & $A_{3}^{\prime}(a)=-0.0119952 \mathrm{~m}^{-1}$ \\
\hline
\end{tabular}



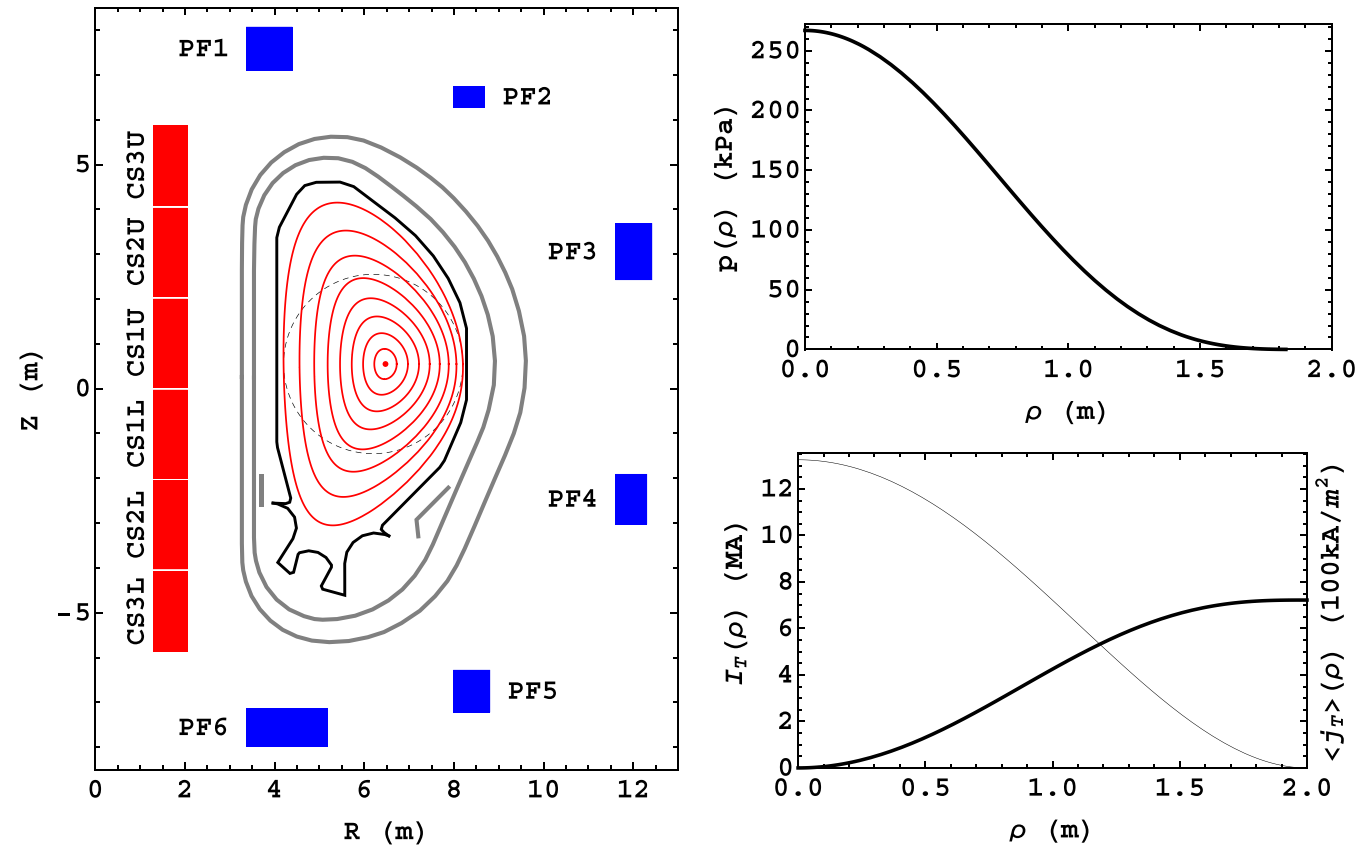

FIG. 2. Left: flux surface geometry inside the ITER-like tokamak plasma. Right: profiles of the plasma pressure, $p(\rho)$, toroidal current, $I_{T}(\rho)$ (thick line), and approximate toroidal current density, $\left\langle j_{T}\right\rangle(\rho)$ (thin line), for the equilibrium detailed in Tables I and III.
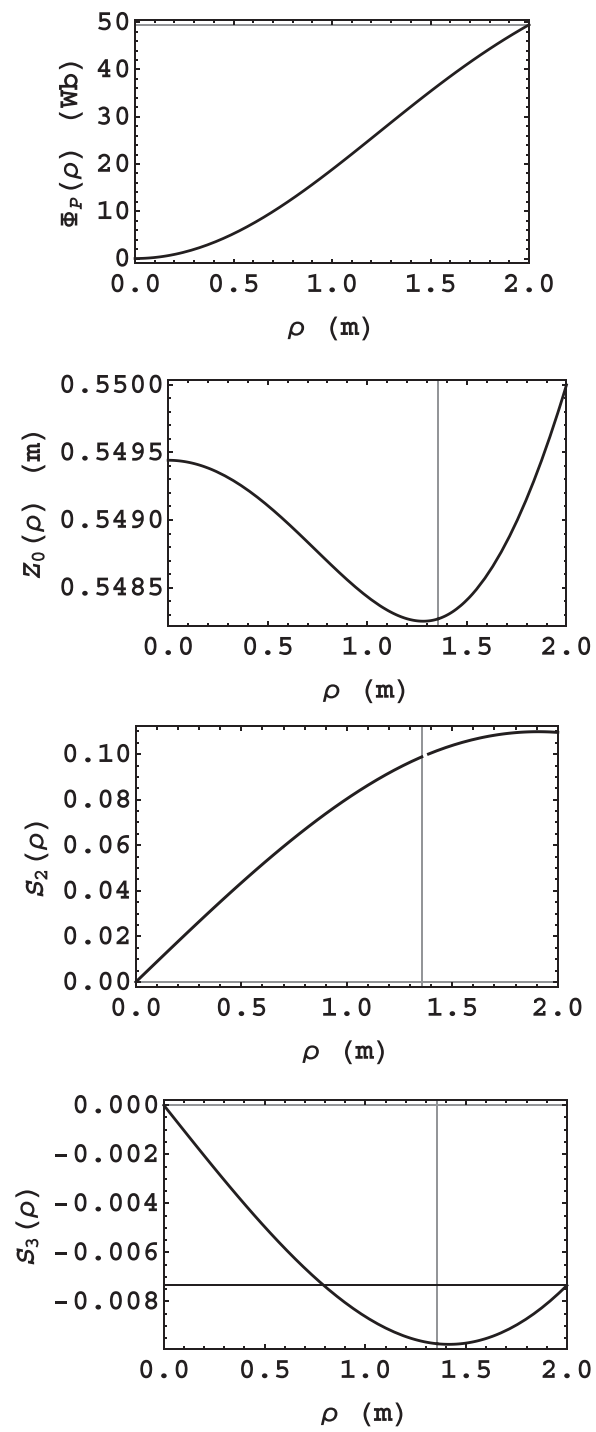
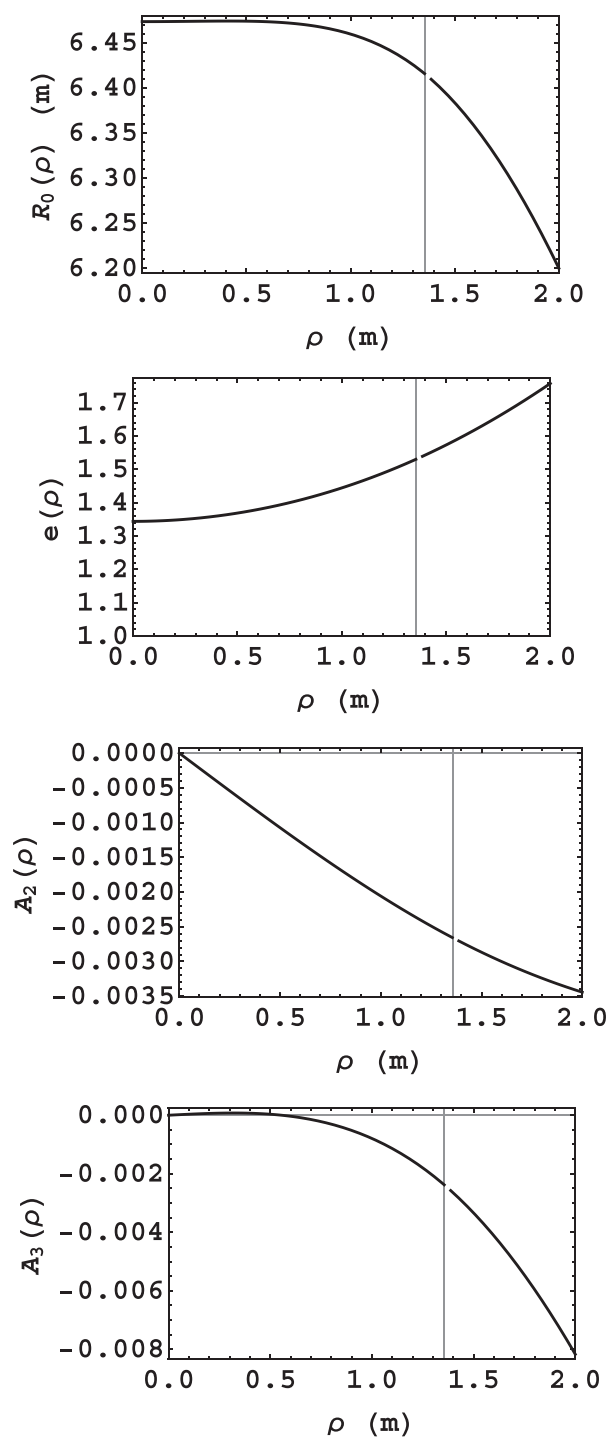

FIG. 3. Profiles of the poloidal flux, $\Phi_{P}(\rho)$; of the main Fourier amplitudes, $R_{0}(\rho), Z_{0}(\rho)$ and $e(\rho)$; of the symmetric, $S_{2}(\rho)$, and antisymmetric, $A_{2}(\rho)$, triangularity coefficients; and of the symmetric, $S_{3}(\rho)$, and antisymmetric, $A_{3}(\rho)$, quadrangularity coefficients for the ITER equilibrium shown in Fig. 2. The thin vertical line indicates the position of the matching radius, $\rho_{*}$. 


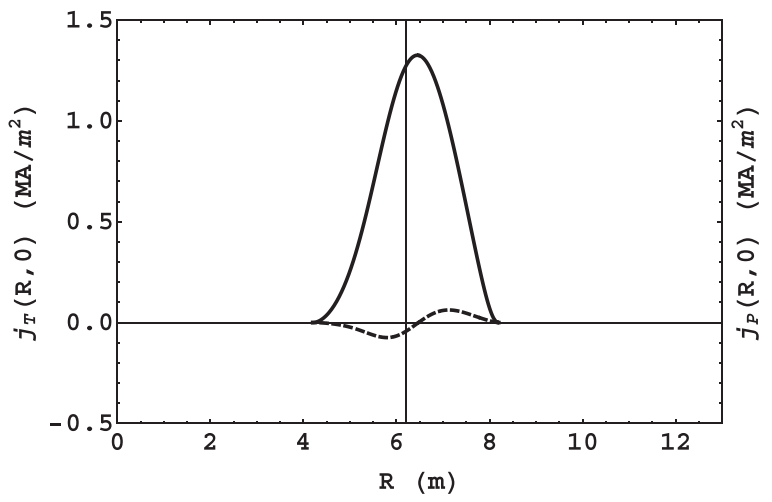

FIG. 4. Profiles of the toroidal (continuous line) and poloidal (dotted line) plasma current densities along the equatorial plane of the ITER equilibrium shown in Fig. 2. The toroidal and poloidal plasma currents are $I_{T}(a)=7.22 \mathrm{MA}$ and $I_{P}(a)=2.82 \mathrm{MA}$, respectively. The total poloidal current is $I(0)=167.12 \mathrm{MA}$ on the magnetic axis and $I(a)=164.30 \mathrm{MA}$ at the plasma boundary.

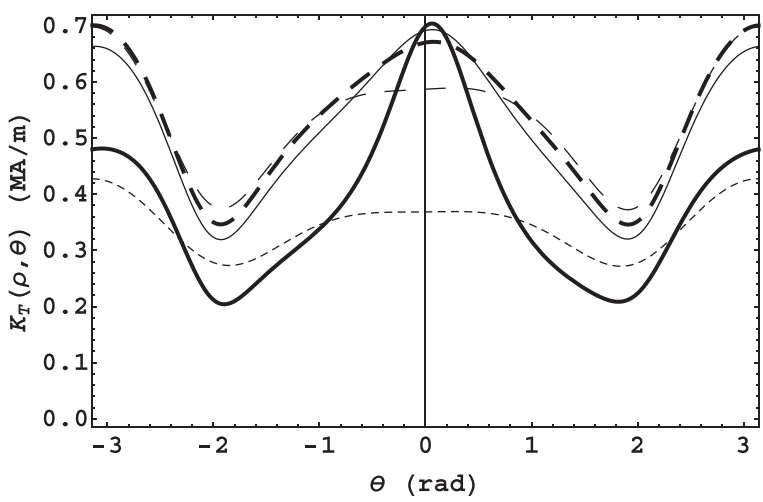

FIG. 5. Equivalent surface current density distribution in the ITER-like tokamak equilibrium shown in Fig. 2. The thick continuous line corresponds to the surface current on the plasma boundary $\rho=a$. The thin lines correspond to the surface current on the flux surfaces $\rho=3 a / 4$ (continuous), $\rho=a / 2$ (dashed), and $\rho=a / 4$ (dotted). The thick dashed line corresponds to the matching radius position $\rho_{*}=0.678 a$.
TABLE IV. Magnetic axis, matching radius and Neumann parameters for the ITER-like tokamak equilibrium with flat pressure and current density profiles shown in Fig. 6, and discussed in the text.

\begin{tabular}{lcc}
\hline \hline Parameter set & Values & \\
\hline Magnetic axis & $R_{\mathrm{m}}=6.24007 \mathrm{~m}$ & $Z_{\mathrm{m}}=0.55123 \mathrm{~m}$ \\
& $\kappa_{m}=1.62433$ & \\
Matching radius & $\rho_{*}=0.576545 \mathrm{~m}$ & \\
Neumann & $R_{0}^{\prime}(a)=-0.0503291$ & $Z_{0}^{\prime}(a)=-0.00116705$ \\
& $e^{\prime}(a)=0.127612 \mathrm{~m}^{-1}$ & \\
& $S_{2}^{\prime}(a)=0.0408533 \mathrm{~m}^{-1}$ & $A_{2}^{\prime}(a)=-0.00161027 \mathrm{~m}^{-1}$ \\
& $S_{3}^{\prime}(a)=0.00494655 \mathrm{~m}^{-1}$ & $A_{3}^{\prime}(a)=-0.00971862 \mathrm{~m}^{-1}$ \\
\hline \hline
\end{tabular}

Upon substituting the values of the Dirichlet parameters, Neumann parameters, and matching radius given in Tables I and III in the sectionally continuous approximations presented in Sec. V, the spectral representation of the flux surfaces is fully determined. Then, the equilibrium quantities can be calculated by integration using the expressions given in Sec. IV. As an example, Fig. 4 shows the profiles, along the equatorial plane, of the toroidal and poloidal current densities defined by Eqs. (29) and (43), respectively.

The toroidal component of the equivalent surface current density, $K_{T}=h_{\zeta} \vec{K} \cdot \nabla \zeta$, is defined on each flux surface of the tokamak plasma by

$$
K_{T}(\rho, \theta)=\frac{|\nabla \rho|}{2 \pi \mu_{0} h_{\zeta}} \frac{d \Phi_{P}}{d \rho}=\frac{1}{\mu_{0}}\left(\frac{h_{\theta}(\rho, \theta)}{2 \pi \sqrt{g}(\rho, \theta)}\right) \frac{I_{T}(\rho)}{K(\rho)} .
$$

Figure 5 shows the surface current density distribution on the plasma boundary and on several flux surfaces inside the plasma for the ITER-like equilibrium shown in Fig. 2. The surface current on the plasma boundary is a necessary part in the determination of the maintaining field. This topic is examined in the Paper II.
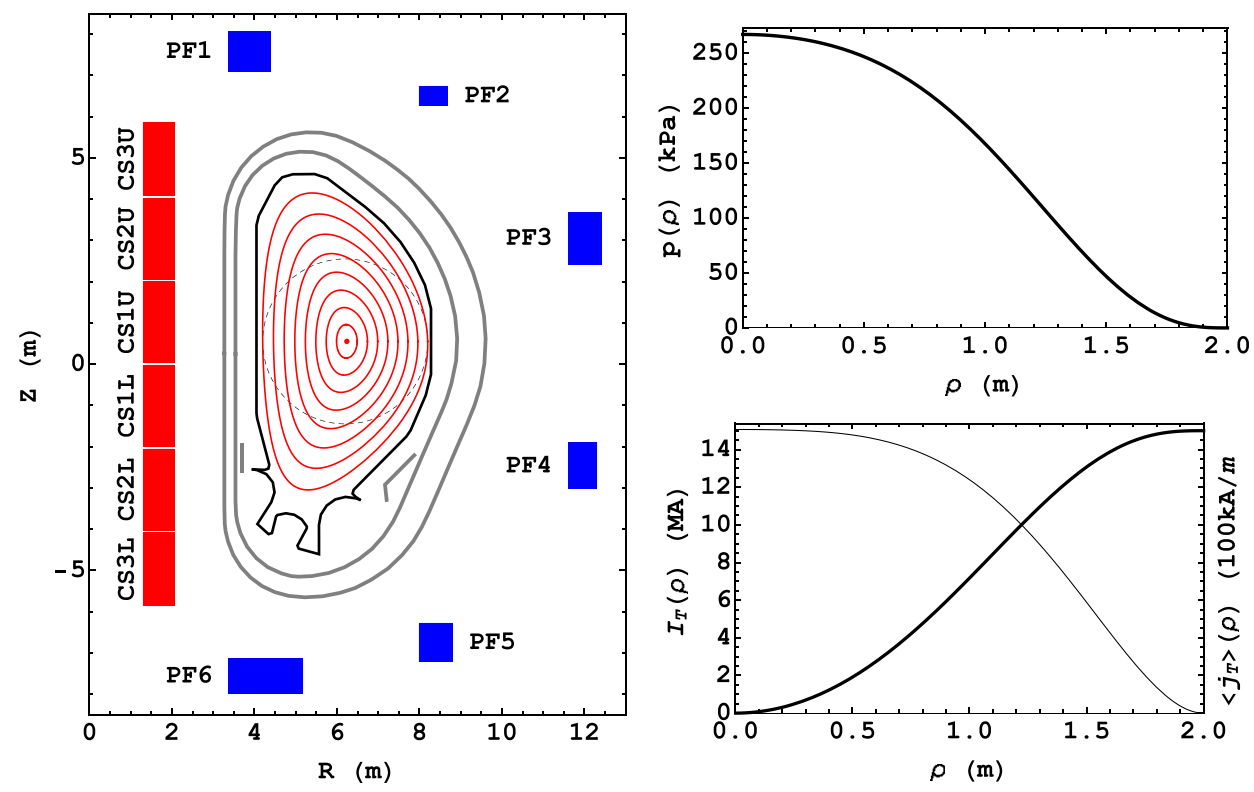

FIG. 6. Left: flux surface geometry inside the ITER-like tokamak plasma. Right: profiles of the plasma pressure, $p(\rho)$, toroidal current, $I_{T}(\rho)$ (thick line), and approximate toroidal current density, $\left\langle j_{T}\right\rangle(\rho)$ (thin line), for the equilibrium detailed in Tables I and IV. 


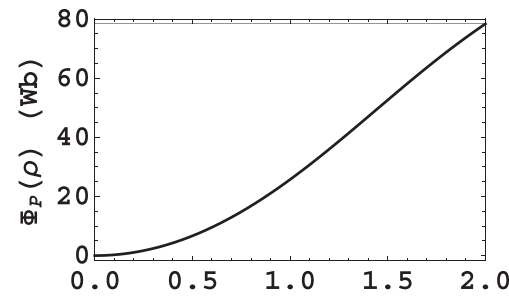

$\rho(\mathrm{m})$

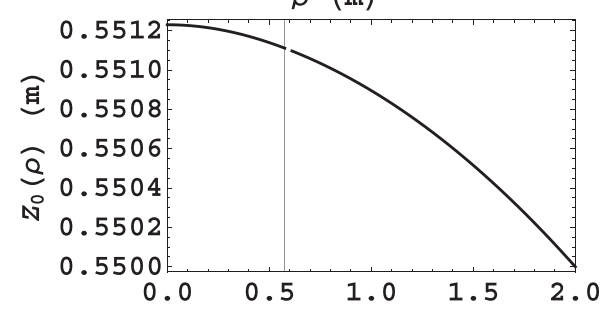

$\rho(\mathrm{m})$

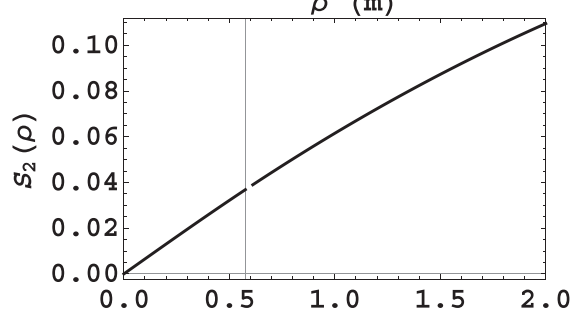

$\rho(\mathrm{m})$

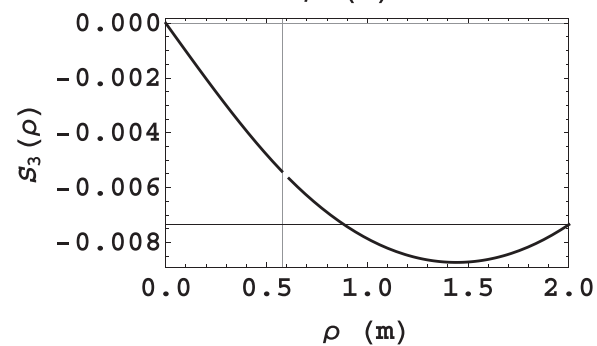

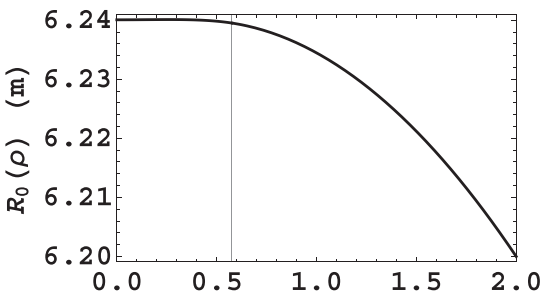

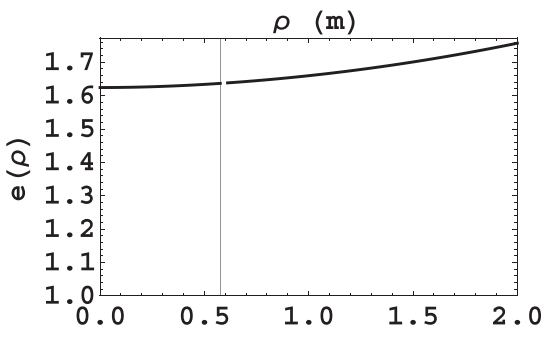

$\rho(\mathrm{m})$

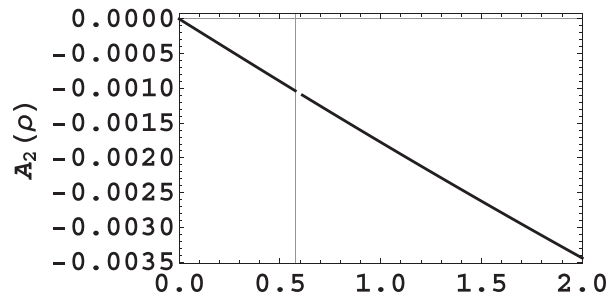

$\rho$ (m)

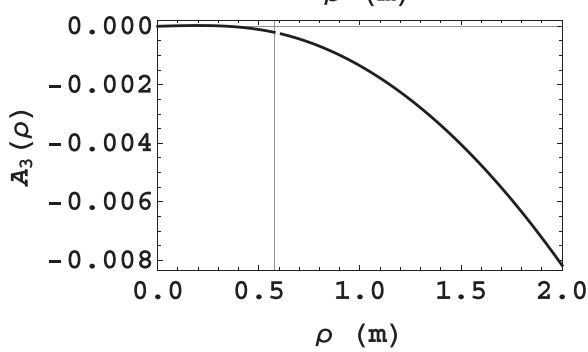

FIG. 7. Profiles of the poloidal flux, $\Phi_{P}(\rho)$; of the main Fourier amplitudes, $R_{0}(\rho), Z_{0}(\rho)$ and $e(\rho)$; of the symmetric, $S_{2}(\rho)$, and antisymmetric, $A_{2}(\rho)$, triangularity coefficients; and of the symmetric, $S_{3}(\rho)$, and antisymmetric, $A_{3}(\rho)$, quadrangularity coefficients for the ITER-like equilibrium shown in Fig. 6 . The thin vertical line indicates the position of the matching radius, $\rho_{*}$.
The last line in Table II corresponds to an ITER-like tokamak equilibrium with relatively flat profiles. Table IV lists the magnetic axis, matching radius, and Neumann parameters for this equilibrium and Fig. 6 shows the flux surface geometry, the plasma pressure profile, $p(\rho)$, the toroidal current profile, $I_{T}(\rho)$, and the approximate toroidal current density profile, $\left\langle j_{T}\right\rangle(\rho)$. Also, Fig. 7 shows the profiles of the poloidal flux and Fourier amplitudes for the equilibrium. Finally, Fig. 8 shows the profiles of the toroidal and poloidal current densities along the equatorial plane, for the ITERlike tokamak equilibrium shown in Figs. 6 and 9 shows the respective distribution of the equivalent surface current density in the internal flux surfaces.

\section{CONCLUSIONS}

This article presented an analytical model for the equilibrium of tokamak plasmas with an arbitrary aspect ratio and vertical asymmetry. The model is based on spectral representations of the flux surfaces and sectionally continuous approximations of the Fourier amplitudes. The coefficients in the sectionally continuous approximations are determined making power series expansions of the variational moment (Euler) equations. Matching between the Taylor series for the core and border plasmas is performed by a direct variational method that admits fast calculation of the equilibrium parameters. In this manner, the analytical form of the spectral representation leads to a straightforward determination of all equilibrium variables and profiles.

The analytical model was applied to an ITER-like tokamak. A wide range of plasma pressure and toroidal current density profiles can be simulated, as listed in Table II and illustrated by Figs. 2 and 6. Although the present application was limited to standard pressure and current (ohmic) profiles, the model can be extended to other profiles including externally driven currents and possibly advanced features such as reversed shear. Even equilibrium solutions with discontinuous profiles (current sheets) can be simulated by suitable modifications of the sectionally continuous approximations.

Although the model may not have sufficient precision for detailed stability studies, when a large number of Fourier components are required, it should be adequate for the conceptual design of tokamak reactors. Moreover, the onedimensional formulation obtained with the use of magnetic 


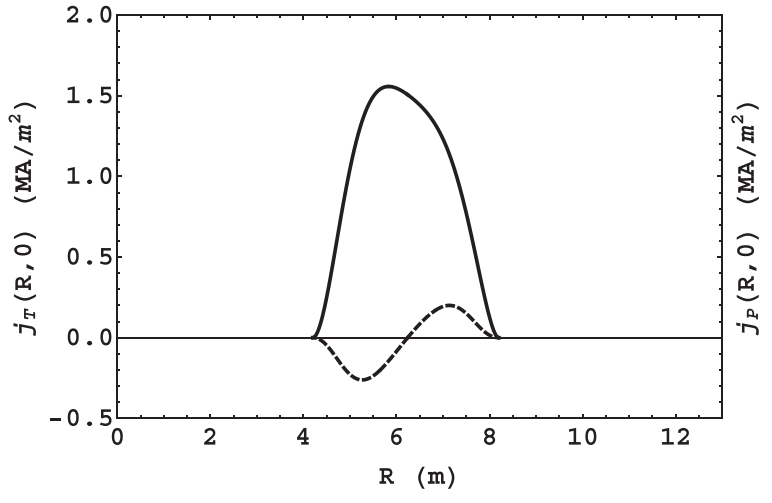

FIG. 8. Profiles of the toroidal (continuous line) and poloidal (dotted line) plasma current densities along the equatorial plane of the ITER-like equilibrium shown in Fig. 6 . The toroidal and poloidal plasma currents are $I_{T}(a)=15.00 \mathrm{MA}$ and $I_{P}(a)=9.78 \mathrm{MA}$, respectively. The total poloidal current is $I(0)=174.08 \mathrm{MA}$ on the magnetic axis and $I(a)=164.30 \mathrm{MA}$ at the plasma boundary.

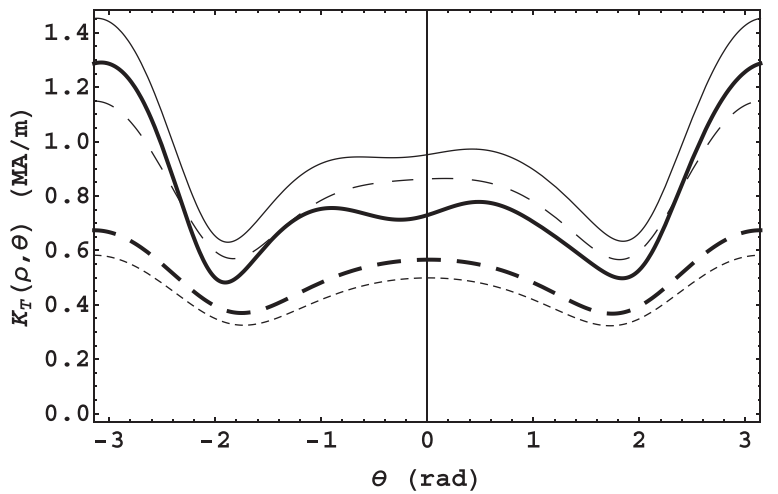

FIG. 9. Equivalent surface current density distribution in the ITER-like tokamak equilibrium shown in Fig. 6. The thick continuous line corresponds to the surface current on the plasma boundary $\rho=a$. The thin lines correspond to the surface current on the flux surfaces $\rho=3 a / 4$ (continuous), $\rho=a / 2$ (dashed), and $\rho=a / 4$ (dotted). The thick dashed line corresponds to the matching radius position $\rho_{*}=0.288 a$.

flux coordinates and spectral representations provides an appropriate framework for simple plasma transport studies and particle orbit calculations, since the flux surfaces have an analytical form. The model is also adequate to estimate the external field necessary for equilibrium, a problem that is addressed in the Paper II. ${ }^{1}$ Actually, one of the advantages of the model is the analytical description of the Cauchy conditions by means of the amplitudes and radial derivatives of the Fourier coefficients at the plasma boundary. The Fourier coefficients act as "virtual filaments" in free-boundary calculations, a problem that is also analyzed in Paper II.

\section{ACKNOWLEDGMENTS}

This work was supported by a grant provided by the Programa Pesquisador Visitante Sênior: Coordenação de Aperfeiçoamento de Pessoal de Nível Superior (CAPES).

\section{APPENDIX A: INTERACTION ENERGY BETWEEN PLASMA AND EXTERNAL TOROIDAL MAGNETIC FIELD}

The vector potential $\vec{A}_{P, 0}$ corresponding to the external toroidal magnetic field $\vec{B}_{T, 0}$ is given by

$$
\vec{A}_{P, 0}=A_{\rho, 0}(\rho, \theta) \nabla \rho+A_{\theta, 0}(\rho, \theta) \nabla \theta
$$

where the covariant components are:

$$
\begin{aligned}
& A_{\rho, 0}(\rho, \theta)=B_{0} R_{0}(a) L(\rho) \frac{\partial}{\partial \rho}\left[\frac{1}{d L / d \rho} \int_{0}^{\theta} \frac{\sqrt{g}\left(\rho, \theta^{\prime}\right)}{h_{\zeta}^{2}\left(\rho, \theta^{\prime}\right)} d \theta^{\prime}\right], \\
& A_{\theta, 0}(\rho, \theta)=B_{0} R_{0}(a) \frac{L(\rho)}{d L / d \rho} \frac{\sqrt{g}(\rho, \theta)}{h_{\zeta}^{2}(\rho, \theta)} .
\end{aligned}
$$

Accordingly,

$$
\begin{aligned}
\nabla \times \vec{A}_{P, 0} & =\nabla A_{\rho, 0}(\rho, \theta) \times \nabla \rho+\nabla A_{\theta, 0}(\rho, \theta) \times \nabla \theta \\
& =\left(-\frac{\partial A_{\rho, 0}}{\partial \theta}+\frac{\partial A_{\theta, 0}}{\partial \rho}\right) \nabla \rho \times \nabla \theta \\
& =\left(-\frac{\partial A_{\rho, 0}}{\partial \theta}+\frac{\partial A_{\theta, 0}}{\partial \rho}\right) \frac{h_{\zeta}^{2}(\rho, \theta)}{\sqrt{g}(\rho, \theta)} \nabla \zeta \\
& =B_{0} R_{0}(a) \nabla \zeta=\vec{B}_{T, 0} .
\end{aligned}
$$

Now consider the interaction energy of the magnetization currents $\overrightarrow{j_{P}}=\nabla \times \vec{M}$ in the external field of potential $\vec{A}_{P, 0}$ :

$$
\begin{aligned}
W_{M}(a) & =\iint_{V(a)} \int_{j_{P}} \cdot \vec{A}_{P, 0} d^{3} r \\
& =\iint_{0}^{a 2 \pi} \frac{d I_{P}}{d \rho} A_{\theta, 0}(\rho, \theta) \underbrace{\nabla \zeta \times \nabla \rho \cdot \nabla \theta \sqrt{g}(\rho, \theta)}_{=1} d \rho d \theta \\
& =2 \pi B_{0} R_{0}(a) \int_{0}^{a} \frac{d I_{P}}{d \rho} \frac{L(\rho)}{d L / d \rho}\left\langle\frac{\sqrt{g}(\rho, \theta)}{h_{\zeta}^{2}(\rho, \theta)}\right\rangle_{\theta} \\
& =I(a) \int_{0}^{a} L(\rho) \frac{d I_{P}}{d \rho} d \rho=L_{M}(a) I(a) I_{P}(a) .
\end{aligned}
$$

Of course, the same result is obtained replacing $\overrightarrow{j_{P}}$ by $\nabla \times \vec{M}:$

$$
\begin{aligned}
W_{M}(a) & =\iiint_{V(a)}(\nabla \times \vec{M}) \cdot \vec{A}_{P, 0} d^{3} r \\
& =\iiint_{V(a)} \vec{M} \cdot \vec{B}_{T, 0} d^{3} r+\iint_{S_{P}(a)} \vec{M} \times \vec{A}_{P, 0} \cdot d^{2} \vec{r}(\rho) .
\end{aligned}
$$

Here, the first term on the right-hand side corresponds to the potential energy of distributed magnetic moments in the external toroidal magnetic field and the second term to the 
interaction energy of surface magnetization. Performing the integrations with $d^{3} r=\sqrt{g}(\rho, \theta) d \rho d \theta d \zeta$ and $d^{2} \vec{r}(\rho)=$ $\sqrt{g}(\rho, \theta) d \theta d \zeta \nabla \rho$ one obtains

$$
\begin{aligned}
W_{M}(a) & =-B_{0} R_{0}(a) \iint_{0}^{a 2 \pi} I_{P}(\rho) \frac{\sqrt{g}(\rho, \theta)}{h_{\zeta}^{2}(\rho, \theta)} d \rho d \theta+I_{P}(a) \int_{0}^{2 \pi}\left[A_{\rho, 0}(a, \theta) \nabla \rho+A_{\theta, 0}(a, \theta) \nabla \theta\right] \times \nabla \zeta \cdot \nabla \rho \sqrt{g}(a, \theta) d \theta \\
& =-\underbrace{\frac{2 \pi B_{0} R_{0}(a)}{\mu_{0}}}_{=I(a)} \int_{0}^{a} I_{P}(\rho) \frac{d L}{d \rho} d \rho+I_{P}(a) \underbrace{\int_{0}^{2 \pi} A_{\theta, 0}(a, \theta) d \theta}_{=L(a) I(a)} \\
& =I(a) \int_{0}^{a} L(\rho) \frac{d I_{P}}{d \rho} d \rho=L_{M}(a) I(a) I_{P}(a) .
\end{aligned}
$$

\section{APPENDIX B: POWER SERIES EXPANSIONS OF THE GEOMETRIC AND MAGNETIC EQUILIBRIUM COEFFICIENTS}

The spectral representation of the flux surfaces expanded to the fourth-order in $\rho$ results in the pair of parametric equations for the radial component (in cylindrical coordinates)

$$
\begin{aligned}
R(\rho, \theta)= & R_{m}+\rho \cos \theta+\frac{\rho^{2}}{2}\left[R_{0}^{\prime \prime}(0)-4\left(S_{2}^{\prime}(0) \sin \theta-A_{2}^{\prime}(0) \cos \theta\right) \sin \theta\right] \\
& -2 \rho^{3}\left(\frac{S_{3}^{\prime}(0)}{a} \sin \theta-\frac{A_{3}^{\prime}(0)}{a} \cos \theta\right) \sin 2 \theta+\frac{\rho^{4}}{24}\left[R_{0}^{(4)}(0)-8\left(S_{2}^{(3)}(0) \sin \theta-A_{2}^{(3)}(0) \cos \theta\right) \sin \theta\right]+\cdots
\end{aligned}
$$

and for the axial component

$$
\begin{aligned}
\frac{Z(\rho, \theta)}{\kappa_{m}}= & \frac{Z_{m}}{\kappa_{m}}+\rho \sin \theta+\frac{\rho^{2}}{2}\left[\frac{Z_{0}^{\prime \prime}(0)}{\kappa_{m}}-4\left(S_{2}^{\prime}(0) \cos \theta+A_{2}^{\prime}(0) \sin \theta\right) \sin \theta\right] \\
& +\frac{\rho^{3}}{2}\left[\frac{e^{\prime \prime}(0)}{\kappa_{m}} \sin \theta-4\left(\frac{S_{3}^{\prime}(0)}{a} \cos \theta+\frac{A_{3}^{\prime}(0)}{a} \sin \theta\right) \sin 2 \theta\right] \\
& +\frac{\rho^{4}}{24}\left[\frac{Z_{0}^{(4)}(0)}{\kappa_{m}}-24 \frac{e^{\prime \prime}(0)}{\kappa_{m}}\left(S_{2}^{\prime}(0) \cos \theta+A_{2}^{\prime}(0) \sin \theta\right) \sin \theta-8\left(S_{2}^{(3)}(0) \cos \theta+A_{2}^{(3)}(0) \sin \theta\right) \sin \theta\right]+\cdots
\end{aligned}
$$

Some of the higher-order terms in the above spectral representation do not contribute to the following expansions of the geometric and magnetic coefficients of the equilibrium equation.

The volume enclosed by a flux surface is expanded as

$$
\frac{V(\rho)}{2 \pi^{2} \kappa_{m} R_{m} \rho^{2}} \cong 1+\frac{\rho^{2}}{2 R_{m}^{2}}\left\{R_{m} R_{0}^{\prime \prime}(0)+\frac{R_{m}^{2} e^{\prime \prime}(0)}{\kappa_{m}}-2\left[1+2 R_{m} S_{2}^{\prime}(0)\right] R_{m} S_{2}^{\prime}(0)-4 R_{m}^{2} A_{2}^{\prime}(0)^{2}-4 \frac{R_{m}^{2} S_{3}^{\prime}(0)}{a}\right\}
$$

and the magnetic coefficients in the equilibrium equation become

$$
\frac{2 R_{m} L(\rho)}{\mu_{0} \kappa_{m} \rho^{2}} \cong 1+\frac{\rho^{2}}{4 R_{m}^{2}}\left\{1-2\left(R_{m} R_{0}^{\prime \prime}(0)-\frac{R_{m}^{2} e^{\prime \prime}(0)}{\kappa_{m}}\right)+4\left[1-2 R_{m} S_{2}^{\prime}(0)\right] R_{m} S_{2}^{\prime}(0)-8\left(R_{m}^{2} A_{2}^{\prime}(0)^{2}+\frac{R_{m}^{2} S_{3}^{\prime}(0)}{a}\right)\right\},
$$

and

$$
\begin{aligned}
\frac{2 \mu_{0} \kappa_{m} R_{m} K(\rho)}{\left(\kappa_{m}^{2}+1\right) \rho} \cong & 1+\frac{\rho^{2}}{4 R_{m}^{2}}\left\{3-\frac{2}{\kappa_{m}^{2}+1}+\left[1-\frac{2}{\kappa_{m}^{2}+1}+\left(3-\frac{2}{\kappa_{m}^{2}+1}\right) R_{m} R_{0}^{\prime \prime}(0)-\frac{16}{\kappa_{m}^{2}+1} R_{m} S_{2}^{\prime}(0)\right] R_{m} R_{0}^{\prime \prime}(0)\right. \\
& +\left[\left(1+\frac{2}{\kappa_{m}^{2}+1}\right) \frac{R_{m} Z_{0}^{\prime \prime}(0)}{\kappa_{m}}+8\left(1-\frac{4}{\kappa_{m}^{2}+1}\right) R_{m} A_{2}^{\prime}(0)\right] \frac{R_{m} Z_{0}^{\prime \prime}(0)}{\kappa_{m}} \\
& +\left(1-\frac{6}{\kappa_{m}^{2}+1}\right) \frac{R_{m}^{2} e^{\prime \prime}(0)}{\kappa_{m}}+4\left[1-\frac{2}{\kappa_{m}^{2}+1}+5\left(1+\frac{2}{\kappa_{m}^{2}+1}\right) R_{m} S_{2}^{\prime}(0)\right] R_{m} S_{2}^{\prime}(0) \\
& \left.+4\left(3+\frac{14}{\kappa_{m}^{2}+1}\right) R_{m}^{2} A_{2}^{\prime}(0)^{2}+8 \frac{R_{m}^{2} S_{3}^{\prime}(0)}{a}\right\} .
\end{aligned}
$$


In a form suitable for a large aspect ratio, the poloidal flux function is given by

$$
\begin{aligned}
\frac{2\left(\kappa_{m}^{2}+1\right) \Phi_{P}[\rho]}{\kappa_{m} \mu_{0} I_{T}^{\prime \prime}(0) R_{m} \rho^{2}} \cong & 1+\frac{\rho^{2}}{24 R_{m}^{2}}\left\{\frac{R_{m}^{2} I_{T}^{(4)}(0)}{I_{T}^{\prime \prime}(0)}-3\left(3-\frac{2}{\kappa_{m}^{2}+1}\right)\right. \\
& -3\left[1-\frac{2}{\kappa_{m}^{2}+1}+\left(3-\frac{2}{\kappa_{m}^{2}+1}\right) R_{m} R_{0}^{\prime \prime}(0)-\frac{16}{\kappa_{m}^{2}+1} R_{m} S_{2}^{\prime}(0)\right] R_{m} R_{0}^{\prime \prime}(0) \\
& -3\left[\left(1+\frac{2}{\kappa_{m}^{2}+1}\right) \frac{R_{m} Z_{0}^{\prime \prime}(0)}{\kappa_{m}}+8\left(1-\frac{4}{\kappa_{m}^{2}+1}\right) R_{m} A_{2}^{\prime}(0)\right] \frac{R_{m} Z_{0}^{\prime \prime}(0)}{\kappa_{m}} \\
& -3\left(1-\frac{6}{\kappa_{m}^{2}+1}\right) \frac{R_{m}^{2} e^{\prime \prime}(0)}{\kappa_{m}}-12\left[1-\frac{2}{\kappa_{m}^{2}+1}+5\left(1+\frac{2}{1+\kappa_{m}^{2}}\right) R_{m} S_{2}^{\prime}(0)\right] R_{m} S_{2}^{\prime}(0) \\
& \left.-12\left[\left(3+\frac{14}{\kappa_{m}^{2}+1}\right) R_{m}^{2} A_{2}^{\prime}(0)^{2}+2 \frac{R_{m}^{2} S_{3}^{\prime}(0)}{a}\right]\right\}
\end{aligned}
$$

Note that the multivariate derivatives $\psi_{m}^{(2,0)}$ and $\psi_{m}^{(4,0)}$ in the coefficients (24) of the Taylor series in $\rho$ of the flux function are related to the derivatives $I_{T}^{\prime \prime}(0)$ and $I_{T}^{(4)}(0)$ of the toroidal current profile, as anticipated in Sec. III.

\section{APPENDIX C: POWER SERIES EXPANSIONS OF THE EULER EQUATIONS}

The leading term (lowest-order) for each Euler equation is as follows.

Geometric center $R_{0}(\rho)$ of the flux surfaces (major radius):

$$
\left(\frac{\left[\kappa_{m}^{2}+R_{m}\left(\left(3 \kappa_{m}^{2}+1\right) R_{0}^{\prime \prime}(0)-8 S_{2}^{\prime}(0)\right)\right] \mu_{0} I_{T}^{\prime \prime}(0)^{2}-8 \pi^{2}\left(\kappa_{m}^{2}+1\right)^{2} p^{\prime \prime}(0)}{2\left(\kappa_{m}^{2}+1\right)^{2}}\right) \kappa_{m} \rho^{3}+\cdots=0 .
$$

Vertical position $Z_{0}(\rho)$ of the major radius:

$$
\left(\frac{R_{m}\left[\left(\kappa_{m}^{2}+3\right) Z_{0}^{\prime \prime}(0) / \kappa_{m}-4\left(3-\kappa_{m}^{2}\right) A_{2}^{\prime}(0)\right] \mu_{0} I_{T}^{\prime \prime}(0)^{2}}{2\left(\kappa_{m}^{2}+1\right)^{2}}\right) \rho^{3}+\cdots=0 .
$$

Elongation $e(\rho)$ :

$$
\begin{aligned}
& \left\{\left[\left(\frac{3 \kappa_{m}^{4}+8 \kappa_{m}^{2}+1-\left(3 \kappa_{m}^{4}+2 \kappa_{m}^{2}+3\right) R_{m} R_{0}^{\prime \prime}(0)+16\left(3 \kappa_{m}^{4}+8 \kappa_{m}^{2}+3\right) R_{m} S_{2}^{\prime}(0)}{16\left(\kappa_{m}^{2}+1\right)^{3}}\right) R_{m} R_{0}^{\prime \prime}(0)\right.\right. \\
& -\left(\frac{\left[21+5 \kappa_{m}^{2}\left(\kappa_{m}^{2}+6\right)\right] R_{m} Z_{0}^{\prime \prime}(0) / \kappa_{m}-16\left(13 \kappa_{m}^{2}+9\right) R_{m} A_{2}^{\prime}(0)}{16\left(\kappa_{m}^{2}+1\right)^{3}}\right) \frac{R_{m} Z_{0}^{\prime \prime}(0)}{\kappa_{m}} \\
& +\left(\frac{\kappa_{m}^{4}+18 \kappa_{m}^{2}+5}{16\left(\kappa_{m}^{2}+1\right)^{3}}\right) \frac{R_{m}^{2} e^{\prime \prime}(0)}{\kappa_{m}}+\left(\frac{1-4 R_{m}\left[5 S_{2}^{\prime}(0)+R_{m}\left(23 S_{2}^{\prime}(0)^{2}+41 A_{2}^{\prime}(0)^{2}-10 S_{3}^{\prime}(0) / a\right)\right]}{16\left(\kappa_{m}^{2}+1\right)^{3}}\right) \\
& +\kappa_{m}^{2}\left(\frac{3+4 R_{m}\left[S_{2}^{\prime}(0)-31 R_{m} S_{2}^{\prime}(0)^{2}-37 R_{m} A_{2}^{\prime}(0)^{2}\right]}{8\left(\kappa_{m}^{2}+1\right)^{3}}\right) \\
& \left.+\kappa_{m}^{4}\left(\frac{1+12 R_{m} S_{2}^{\prime}(0)-4 R_{m}^{2}\left[19 S_{2}^{\prime}(0)^{2}+5 A_{2}^{\prime}(0)^{2}+10 S_{3}^{\prime}(0) / a\right]}{16\left(\kappa_{m}^{2}+1\right)^{3}}\right)\right] \mu_{0} I_{T}^{\prime \prime}(0)^{2} \\
& \left.+\left(\frac{\left(\kappa_{m}^{2}-1\right) R_{m}^{2}}{48\left(\kappa_{m}^{2}+1\right)^{2}}\right) \mu_{0} I_{T}^{\prime \prime}(0) I_{T}^{(4)}(0)-\left(\frac{\pi^{2}\left[1-4 R_{m}\left(R_{0}^{\prime \prime}(0)-2 S_{2}^{\prime}(0)\right)\right]}{2}\right) p^{\prime \prime}(0)\right\} \frac{\rho^{5}}{R_{m}}+\cdots=0 .
\end{aligned}
$$


Symmetric triangularity coefficient $S_{2}(\rho)$ :

$$
-\left(\frac{\left[\kappa_{m}^{2}+R_{m}\left(\left(3 \kappa_{m}^{2}+1\right) R_{0}^{\prime \prime}(0)-8 S_{2}^{\prime}(0)\right)\right] \mu_{0} I_{T}^{\prime \prime}(0)^{2}-8 \pi^{2}\left(\kappa_{m}^{2}+1\right)^{2} p^{\prime \prime}(0)}{2\left(\kappa_{m}^{2}+1\right)^{2}}\right) \kappa_{m} \rho^{4}+\cdots=0 .
$$

Antisymmetric triangularity coefficient $A_{2}(\rho)$ :

$$
-\left(\frac{R_{m}\left[\left(\kappa_{m}^{2}+3\right) Z_{0}^{\prime \prime}(0) / \kappa_{m}-4\left(3-\kappa_{m}^{2}\right) A_{2}^{\prime}(0)\right] \mu_{0} I_{T}^{\prime \prime}(0)^{2}}{2\left(\kappa_{m}^{2}+1\right)^{2}}\right) \kappa_{m} \rho^{4}+\cdots=0
$$

Symmetric quadrangular coefficient $S_{3}(\rho)$ :

$$
\begin{aligned}
& -\left\{\left[\left(\frac{2\left(13 \kappa_{m}^{2}+7\right)+\left(57 \kappa_{m}^{2}+47\right) R_{m} R_{0}^{\prime \prime}(0)-4\left(43 \kappa_{m}^{2}+141\right) R_{m} S_{2}^{\prime}(0)}{32\left(\kappa_{m}^{2}+1\right)^{2}}\right) R_{m} R_{0}^{\prime \prime}(0)\right.\right. \\
& +\left(\frac{\left(47 \kappa_{m}^{2}+57\right) R_{m} Z_{0}^{\prime \prime}(0) / \kappa_{m}+4\left(47 \kappa_{m}^{2}-71\right) R_{m} A_{2}^{\prime}(0)}{32\left(\kappa_{m}^{2}+1\right)^{2}}\right) \frac{R_{m} Z_{0}^{\prime \prime}(0)}{\kappa_{m}} \\
& -\left(\frac{7\left(\kappa_{m}^{2}+7\right)}{16\left(\kappa_{m}^{2}+1\right)^{2}}\right) \frac{R_{m}^{2} e^{\prime \prime}(0)}{\kappa_{m}}+\left(\frac{21-238 R_{m} S_{2}^{\prime}(0)+4 R_{m}^{2}\left(495 S_{2}^{\prime}(0)^{2}+273 A_{2}^{\prime}(0)^{2}+154 S_{3}^{\prime}(0) / a\right)}{32\left(\kappa_{m}^{2}+1\right)^{2}}\right) \\
& \left.+\kappa_{m}^{2}\left(\frac{35-34 R_{m} S_{2}^{\prime}(0)+28 R_{m}^{2}\left(31 S_{2}^{\prime}(0)^{2}+17 A_{2}^{\prime}(0)^{2}+22 S_{3}^{\prime}(0) / a\right)}{32\left(\kappa_{m}^{2}+1\right)^{2}}\right)\right] \mu_{0} I_{T}^{\prime \prime}(0)^{2} \\
& \left.+4 \pi^{2} R_{m}\left(R_{0}^{\prime \prime}(0)-4 S_{2}^{\prime}(0)\right) p^{\prime \prime}(0)\right\} \frac{\kappa_{m} \rho^{6}}{R_{m} a}+\cdots=0 .
\end{aligned}
$$

Antisymmetric quadrangular coefficient $A_{3}(\rho)$ :

$$
\begin{aligned}
& \left\{\left[\left(\frac{7\left(\kappa_{m}^{2}-1\right)+21\left(\kappa_{m}^{2}-1\right) R_{m} R_{0}^{\prime \prime}(0)+2\left(13 \kappa_{m}^{2}+67\right) R_{m} S_{2}^{\prime}(0)}{16\left(\kappa_{m}^{2}+1\right)^{2}}\right) \frac{R_{m} Z_{0}^{\prime \prime}(0)}{\kappa_{m}}\right.\right. \\
& \quad+\left(\frac{19 \kappa_{m}^{2}+77+10\left(5 \kappa_{m}^{2}+11\right) R_{m} R_{0}^{\prime \prime}(0)-4\left(23 \kappa_{m}^{2}+137\right) R_{m} S_{2}^{\prime}(0)}{16\left(\kappa_{m}^{2}+1\right)^{2}}\right) R_{m} A_{2}^{\prime}(0) \\
& \left.\left.\quad-\left(\frac{35 R_{m}^{2} A_{3}^{\prime}(0) / a}{4\left(\kappa_{m}^{2}+1\right)}\right)\right] \mu_{0} I_{T}^{\prime \prime}(0)^{2}+8 \pi^{2} R_{m} A_{2}^{\prime}(0) p^{\prime \prime}(0)\right\} \frac{\kappa_{m} \rho^{6}}{R_{m} a}+\cdots=0
\end{aligned}
$$

Note that the two equations for the symmetric and antisymmetric triangularity coefficients give lowest-order conditions that are identical to the ones given by the radial and vertical positions of the major radius. Therefore, in lowest-order there are five independent equations available, which can be used to evaluate the three parameters $R_{m}, Z_{m}$, and $\kappa_{m}$ on the magnetic axis, and to eliminate, say, $S_{3}^{\prime}(0)$ and $A_{3}^{\prime}(0)$. The remaining coefficients, $R_{0}^{\prime \prime}(0), Z_{0}^{\prime \prime}(0), e^{\prime \prime}(0), S_{2}^{\prime}(0)$, and $A_{2}^{\prime}(0)$, must be evaluated from the next order terms in the expansions of the Euler equations. These terms become very cumbersome. Nevertheless, they can be derived from the Euler equations in a straightforward manner using a computer algebra system like Mathematica. Although lengthy, the final equations have a simple algebraic structure, allowing fast calculation of the required coefficients. The seven equations suitable for evaluating the coefficients $R_{0}^{\prime \prime}(0), Z_{0}^{\prime \prime}(0), e^{\prime \prime}(0), S_{2}^{\prime}(0), A_{2}^{\prime}(0), S_{3}^{\prime}(0)$, and $A_{3}^{\prime}(0)$ are listed in the following. They complement the three Eqs. (76)-(78) used to evaluate $R_{m}, Z_{m}$, and $\kappa_{m}$.

Next to the lowest order term for the major radius $R_{0}(\rho)$ of the flux surfaces: 


$$
\begin{aligned}
& 3\left(5 \kappa_{m}^{2}-13+\frac{12}{\kappa_{m}^{2}+1}\right)-6\left(3 \kappa_{m}^{2}+1\right) R_{m}^{3} R_{0}^{(4)}(0)-3\left[6\left(\kappa_{m}^{2}+5-\frac{4}{\kappa_{m}^{2}+1}\right) R_{m}^{2} R_{0}^{\prime \prime}(0)^{2}\right. \\
& \left.+\left(18 \kappa_{m}^{2}-6+\frac{24}{\kappa_{m}^{2}+1}\right) \frac{R_{m}^{2} Z_{0}^{\prime \prime}(0)^{2}}{\kappa_{m}^{2}}\right] R_{m} R_{0}^{\prime \prime}(0)+3\left[15 \kappa_{m}^{2}-43+\frac{36}{\kappa_{m}^{2}+1}-288\left(1-\frac{1}{\kappa_{m}^{2}+1}\right) R_{m} S_{2}^{\prime}(0)\right] R_{m}^{2} R_{0}^{\prime \prime}(0)^{2} \\
& -3\left[\kappa_{m}^{2}-9+\frac{12}{\kappa_{m}^{2}+1}-48\left(3 \kappa_{m}^{2}+5-\frac{2}{\kappa_{m}^{2}+1}\right) R_{m} S_{2}^{\prime}(0)\right] \frac{R_{m}^{2} Z_{0}^{\prime \prime}(0)^{2}}{\kappa_{m}^{2}} \\
& -18\left[8\left(3 \kappa_{m}^{2}+11-\frac{8}{\kappa_{m}^{2}+1}\right) R_{m} A_{2}^{\prime}(0) \frac{R_{m} Z_{0}^{\prime \prime}(0)}{\kappa_{m}}-\left(3 \kappa_{m}^{2}-13+\frac{12}{\kappa_{m}^{2}+1}\right) \frac{R_{m}^{2} e^{\prime \prime}(0)}{\kappa_{m}}\right] R_{m} R_{0}^{\prime \prime}(0) \\
& +3\left[49 \kappa_{m}^{2}-31+\frac{36}{\kappa_{m}^{2}+1}+8\left(2\left(3 \kappa_{m}^{2}-17+\frac{18}{\kappa_{m}^{2}+1}\right)+9\left(\kappa_{m}^{2}+1+\frac{4}{\kappa_{m}^{2}+1}\right) R_{m} S_{2}^{\prime}(0)\right) R_{m} S_{2}^{\prime}(0)\right. \\
& \left.-24\left(3 \kappa_{m}^{2}-37+\frac{28}{\kappa_{m}^{2}+1}\right) R_{m}^{2} A_{2}^{\prime}(0)^{2}\right] R_{m} R_{0}^{\prime \prime}(0)-24\left[\kappa_{m}^{2}+27-\frac{24}{\kappa_{m}^{2}+1}-12\left(3 \kappa_{m}^{2}-13+\frac{16}{\kappa_{m}^{2}+1}\right) R_{m} S_{2}^{\prime}(0)\right] R_{m} A_{2}^{\prime}(0) \frac{R_{m} Z_{0}^{\prime \prime}(0)}{\kappa_{m}} \\
& +3\left[\kappa_{m}^{2}-41+\frac{36}{\kappa_{m}^{2}+1}-48\left(\kappa_{m}^{2}+5-\frac{6}{\kappa_{m}^{2}+1}\right) R_{m} S_{2}^{\prime}(0)\right] \frac{R_{m}^{2} e^{\prime \prime}(0)}{\kappa_{m}} \\
& +12\left[\kappa_{m}^{2}-43+\frac{36}{\kappa_{m}^{2}+1}+\left(29 \kappa_{m}^{2}+19+\frac{36}{\kappa_{m}^{2}+1}+24\left(5 \kappa_{m}^{2}+7-\frac{20}{\kappa_{m}^{2}+1}\right) R_{m} S_{2}^{\prime}(0)\right) R_{m} S_{2}^{\prime}(0)\right] R_{m} S_{2}^{\prime}(0) \\
& +12\left[19 \kappa_{m}^{2}+101-\frac{84}{\kappa_{m}^{2}+1}+24\left(3 \kappa_{m}^{2}+13-\frac{28}{\kappa_{m}^{2}+1}\right) R_{m} S_{2}^{\prime}(0)\right] R_{m}^{2} A_{2}^{\prime}(0)^{2}+24\left(\kappa_{m}^{2}+3\right) R_{m}^{3} S_{2}^{(3)}(0) \\
& +24\left[7 \kappa_{m}^{2}-5-24 R_{m} R_{0}^{\prime \prime}(0)+12\left(7 \kappa_{m}^{2}+9\right) R_{m} S_{2}^{\prime}(0)\right] \frac{R_{m}^{2} S_{3}^{\prime}(0)}{a}+144\left[3\left(\kappa_{m}^{2}-1\right) \frac{R_{m} Z_{0}^{\prime \prime}(0)}{\kappa_{m}}+8\left(\kappa_{m}^{2}+2\right) R_{m} A_{2}^{\prime}(0)\right] \frac{R_{m}^{2} A_{3}^{\prime}(0)}{a} \\
& +\left[5 \kappa_{m}^{2}-1+\left(18 \kappa_{m}^{2}+6\right) R_{m} R_{0}^{\prime \prime}(0)-48 R_{m} S_{2}^{\prime}(0)\right] \frac{R_{m}^{2} I_{T}^{(4)}(0)}{I_{T}^{\prime \prime}(0)}+4 \kappa_{m}^{2}\left(\frac{8 \pi^{2}\left(\kappa_{m}^{2}+1\right)^{2} R_{m}^{2} p^{(4)}(0)}{\kappa_{m}^{2} \mu_{0} I_{T}^{\prime \prime}(0)^{2}}\right) \\
& +3 \kappa_{m}^{2}\left[1+4\left(R_{m} R_{0}^{\prime \prime}(0)-2 R_{m} S_{2}^{\prime}(0)+\frac{R_{m}^{2} e^{\prime \prime}(0)}{\kappa_{m}}-4\left(R_{m}^{2} S_{2}^{\prime}(0)^{2}+R_{m}^{2} A_{2}^{\prime}(0)^{2}+\frac{R_{m}^{2} S_{3}^{\prime}(0)}{a}\right)\right)\right]\left(\frac{8 \pi^{2}\left(\kappa_{m}^{2}+1\right)^{2} p^{\prime \prime}(0)}{\kappa_{m}^{2} \mu_{0} I_{T}^{\prime \prime}(0)^{2}}\right)=0 .
\end{aligned}
$$

Next to the lowest order term for the vertical position $Z_{0}(\rho)$ of the major radius:

$$
\begin{aligned}
& \left(\kappa_{m}^{2}+3\right) \frac{R_{m}^{3} Z_{0}^{(4)}(0)}{\kappa_{m}}+3\left[\left(3 \kappa_{m}^{2}-1+\frac{4}{\kappa_{m}^{2}+1}\right) R_{m}^{2} R_{0}^{\prime \prime}(0)^{2}+\left(\kappa_{m}^{2}+5-\frac{4}{\kappa_{m}^{2}+1}\right) \frac{R_{m}^{2} Z_{0}^{\prime \prime}(0)^{2}}{\kappa_{m}^{2}}\right] \frac{R_{m} Z_{0}^{\prime \prime}(0)}{\kappa_{m}} \\
& +12\left[\left(3 \kappa_{m}^{2}+11-\frac{8}{\kappa_{m}^{2}+1}\right) R_{m}^{2} R_{0}^{\prime \prime}(0)^{2}-3\left(\kappa_{m}^{2}+9-\frac{8}{\kappa_{m}^{2}+1}\right) \frac{R_{m}^{2} Z_{0}^{\prime \prime}(0)^{2}}{\kappa_{m}^{2}}\right] R_{m} A_{2}^{\prime}(0) \\
& +3\left[\left(\kappa_{m}^{2}-1+\frac{4}{\kappa_{m}^{2}+1}-16\left(3 \kappa_{m}^{2}+5-\frac{2}{\kappa_{m}^{2}+1}\right) R_{m} S_{2}^{\prime}(0)\right) R_{m} R_{0}^{\prime \prime}(0)-\left(5 \kappa_{m}^{2}+21-\frac{12}{\kappa_{m}^{2}+1}\right) \frac{\left.R_{m}^{2} e^{\prime \prime}(0)\right]}{\kappa_{m}}\right] \frac{R_{m} Z_{0}^{\prime \prime}(0)}{\kappa_{m}} \\
& +12\left[\kappa_{m}^{2}+7-\frac{8}{\kappa_{m}^{2}+1}-4\left(3 \kappa_{m}^{2}-13+\frac{16}{\kappa_{m}^{2}+1}\right) R_{m} S_{2}^{\prime}(0)\right] R_{m} A_{2}^{\prime}(0) R_{m} R_{0}^{\prime \prime}(0) \\
& -6\left[\kappa_{m}^{2}+3-\frac{2}{\kappa_{m}^{2}+1}+2\left(4\left(\kappa_{m}^{2}+1-\frac{1}{\kappa_{m}^{2}+1}\right)-\left(27 \kappa_{m}^{2}+59-\frac{20}{\kappa_{m}^{2}+1}\right) R_{m} S_{2}^{\prime}(0)\right) R_{m} S_{2}^{\prime}(0)\right. \\
& \left.+6\left(\kappa_{m}^{2}-55+\frac{52}{\kappa_{m}^{2}+1}\right) R_{m}^{2} A_{2}^{\prime}(0)^{2}\right] \frac{R_{m} Z_{0}^{\prime \prime}(0)}{\kappa_{m}}-24\left(\kappa_{m}^{2}-14+\frac{12}{\kappa_{m}^{2}+1}\right) R_{m} A_{2}^{\prime}(0) \frac{R_{m}^{2} e^{\prime \prime}(0)}{\kappa_{m}} \\
& -24\left[\kappa_{m}^{2}-5+\frac{4}{\kappa_{m}^{2}+1}+\left(2 \kappa_{m}^{2}-11+\frac{16}{\kappa_{m}^{2}+1}+4\left(3 \kappa_{m}^{2}+20-\frac{20}{\kappa_{m}^{2}+1}\right) R_{m} S_{2}^{\prime}(0)\right) R_{m} S_{2}^{\prime}(0)\right. \\
& \left.+4\left(\kappa_{m}^{2}+26-\frac{28}{\kappa_{m}^{2}+1}\right) R_{m}^{2} A_{2}^{\prime}(0)^{2}\right] R_{m} A_{2}^{\prime}(0)+4\left(\kappa_{m}^{2}-5\right) R_{m}^{3} A_{2}^{(3)}(0)+48\left[2 \kappa_{m}^{2} \frac{R_{m} Z_{0}^{\prime \prime}(0)}{\kappa_{m}}+\left(\kappa_{m}^{2}+7\right) R_{m} A_{2}^{\prime}(0)\right] \frac{R_{m}^{2} S_{3}^{\prime}(0)}{a} \\
& -24\left[\left(\kappa_{m}^{2}-1\right)\left(1+3 R_{m} R_{0}^{\prime \prime}(0)\right)+4\left(\kappa_{m}^{2}+5\right) R_{m} S_{2}^{\prime}(0)\right] \frac{R_{m}^{2} A_{3}^{\prime}(0)}{a}+\left(\left(\kappa_{m}^{2}+3\right) \frac{R_{m} Z_{0}^{\prime \prime}(0)}{\kappa_{m}}+4\left(\kappa_{m}^{2}-3\right) R_{m} A_{2}^{\prime}(0)\right) \frac{R_{m}^{2} I_{T}^{(4)}(0)}{I_{T}^{\prime \prime}(0)}=0 .
\end{aligned}
$$


Note that the last term in the above equation vanishes as determined by Eq. (77) for the vertical position of the magnetic axis.

Next to the lowest order term for the elongation $e(\rho)$ :

$$
\begin{aligned}
& 45\left(11 \kappa_{m}^{2}+5+\frac{28}{\kappa_{m}^{2}+1}-\frac{24}{\left(\kappa_{m}^{2}+1\right)^{2}}\right)+60\left[5 \kappa_{m}^{2}+6-\frac{4}{\kappa_{m}^{2}+1}+\left(9 \kappa_{m}^{2}+17-\frac{8}{\kappa_{m}^{2}+1}\right) R_{m} R_{0}^{\prime \prime}(0)\right. \\
& \left.-8\left(5+\frac{4}{\kappa_{m}^{2}+1}\right) R_{m} S_{2}^{\prime}(0)\right] R_{m}^{3} R_{0}^{(4)}(0)+60\left[\left(5 \kappa_{m}^{2}+25+\frac{8}{\kappa_{m}^{2}+1}\right) \frac{R_{m} Z_{0}^{\prime \prime}(0)}{\kappa_{m}}+8\left(2 \kappa_{m}^{2}-7-\frac{8}{\kappa_{m}^{2}+1}\right) R_{m} A_{2}^{\prime}(0)\right] \frac{R_{m}^{3} Z_{0}^{(4)}(0)}{\kappa_{m}} \\
& -60\left(3 \kappa_{m}^{2}+15+\frac{8}{\kappa_{m}^{2}+1}\right) \frac{R_{m}^{4} e^{(4)}(0)}{\kappa_{m}}+45\left(57 \kappa_{m}^{2}+23+\frac{28}{\kappa_{m}^{2}+1}-\frac{24}{\left(\kappa_{m}^{2}+1\right)^{2}}\right) R_{m}^{4} R_{0}^{\prime \prime}(0)^{4} \\
& +45\left(29 \kappa_{m}^{2}+203+\frac{140}{\kappa_{m}^{2}+1}-\frac{24}{\left(\kappa_{m}^{2}+1\right)^{2}}\right) \frac{R_{m}^{4} Z_{0}^{\prime \prime}(0)^{4}}{\kappa_{m}^{4}}+270\left(19 \kappa_{m}^{2}+57-\frac{28}{\kappa_{m}^{2}+1}+\frac{8}{\left(\kappa_{m}^{2}+1\right)^{2}}\right) R_{m}^{2} R_{0}^{\prime \prime}(0)^{2} \frac{R_{m}^{2} Z_{0}^{\prime \prime}(0)^{2}}{\kappa_{m}^{2}} \\
& +270\left[3 \kappa_{m}^{2}-5+\frac{12}{\kappa_{m}^{2}+1}-\frac{8}{\left(\kappa_{m}^{2}+1\right)^{2}}-32\left(2 \kappa_{m}^{2}+4-\frac{3}{\kappa_{m}^{2}+1}+\frac{2}{\left(\kappa_{m}^{2}+1\right)^{2}}\right) R_{m} S_{2}^{\prime}(0)\right] R_{m}^{3} R_{0}^{\prime \prime}(0)^{3} \\
& +1440\left(4 \kappa_{m}^{2}-55-\frac{116}{\kappa_{m}^{2}+1}+\frac{24}{\left(\kappa_{m}^{2}+1\right)^{2}}\right) R_{m} A_{2}^{\prime}(0) \frac{R_{m}^{3} Z_{0}^{\prime \prime}(0)^{3}}{\kappa_{m}^{3}} \\
& +1440\left(15 \kappa_{m}^{2}-56+\frac{60}{\kappa_{m}^{2}+1}-\frac{24}{\left(\kappa_{m}^{2}+1\right)^{2}}\right) R_{m} A_{2}^{\prime}(0) R_{m}^{2} R_{0}^{\prime \prime}(0)^{2} \frac{R_{m} Z_{0}^{\prime \prime}(0)}{\kappa_{m}}+90\left[17 \kappa_{m}^{2}+25-\frac{76}{\kappa_{m}^{2}+1}+\frac{24}{\left(\kappa_{m}^{2}+1\right)^{2}}\right. \\
& \left.-16\left(19 \kappa_{m}^{2}+77+\frac{46}{\kappa_{m}^{2}+1}-\frac{12}{\left(\kappa_{m}^{2}+1\right)^{2}}\right) R_{m} S_{2}^{\prime}(0)\right] R_{m} R_{0}^{\prime \prime}(0) \frac{R_{m}^{2} Z_{0}^{\prime \prime}(0)^{2}}{\kappa_{m}^{2}} \\
& -180\left[\left(9 \kappa_{m}^{2}+72-\frac{68}{\kappa_{m}^{2}+1}+\frac{36}{\left(\kappa_{m}^{2}+1\right)^{2}}\right) R_{m}^{2} R_{0}^{\prime \prime}(0)^{2}+\left(12 \kappa_{m}^{2}+133+\frac{152}{\kappa_{m}^{2}+1}-\frac{36}{\left(\kappa_{m}^{2}+1\right)^{2}}\right) \frac{R_{m}^{2} Z_{0}^{\prime \prime}(0)^{2}}{\kappa_{m}^{2}}\right] \frac{R_{m}^{2} e^{\prime \prime}(0)}{\kappa_{m}} \\
& +45\left[17 \kappa_{m}^{2}-57+\frac{148}{\kappa_{m}^{2}+1}-\frac{72}{\left(\kappa_{m}^{2}+1\right)^{2}}-8\left(25 \kappa_{m}^{2}-9-\frac{84}{1+\kappa_{m}^{2}}+\frac{72}{\left(1+\kappa_{m}^{2}\right)^{2}}\right.\right. \\
& \left.\left.-\left(147 \kappa_{m}^{2}+657+\frac{100}{\kappa_{m}^{2}+1}-\frac{72}{\left(\kappa_{m}^{2}+1\right)^{2}}\right) R_{m} S_{2}^{\prime}(0)\right) R_{m} S_{2}^{\prime}(0)+8\left(81 \kappa_{m}^{2}+451-\frac{404}{\kappa_{m}^{2}+1}+\frac{168}{\left(\kappa_{m}^{2}+1\right)^{2}}\right) R_{m}^{2} A_{2}^{\prime}(0)^{2}\right] R_{m}^{2} R_{0}^{\prime \prime}(0)^{2} \\
& +90\left[25 \kappa_{m}^{2}+95-\frac{84}{\kappa_{m}^{2}+1}+\frac{24}{\left(\kappa_{m}^{2}+1\right)^{2}}-4\left(13 \kappa_{m}^{2}+23+\frac{52}{1+\kappa_{m}^{2}}-\frac{24}{\left(1+\kappa_{m}^{2}\right)^{2}}\right.\right. \\
& \left.-\left(227 \kappa_{m}^{2}+1109+\frac{532}{\kappa_{m}^{2}+1}-\frac{120}{\left(\kappa_{m}^{2}+1\right)^{2}}\right) R_{m} S_{2}^{\prime}(0)\right) R_{m} S_{2}^{\prime}(0) \\
& \left.+4\left(81 \kappa_{m}^{2}+655+\frac{3740}{\kappa_{m}^{2}+1}-\frac{936}{\left(\kappa_{m}^{2}+1\right)^{2}}\right) R_{m}^{2} A_{2}^{\prime}(0)^{2}\right] \frac{R_{m}^{2} Z_{0}^{\prime \prime}(0)^{2}}{\kappa_{m}^{2}}+45\left(27 \kappa_{m}^{2}+241+\frac{468}{\kappa_{m}^{2}+1}-\frac{216}{\left(\kappa_{m}^{2}+1\right)^{2}}\right) \frac{R_{m}^{4} e^{\prime \prime}(0)^{2}}{\kappa_{m}^{2}} \\
& +1440\left[4 \kappa_{m}^{2}-11+\frac{56}{\kappa_{m}^{2}+1}-\frac{24}{\left(\kappa_{m}^{2}+1\right)^{2}}-4\left(14 \kappa_{m}^{2}-37-\frac{136}{\kappa_{m}^{2}+1}+\frac{48}{\left(\kappa_{m}^{2}+1\right)^{2}}\right) R_{m} S_{2}^{\prime}(0)\right] R_{m} A_{2}^{\prime}(0) R_{m} R_{0}^{\prime \prime}(0) \frac{R_{m} Z_{0}^{\prime \prime}(0)}{\kappa_{m}} \\
& -180\left[10 \kappa_{m}^{2}+11-\frac{56}{\kappa_{m}^{2}+1}+\frac{36}{\left(\kappa_{m}^{2}+1\right)^{2}}-32\left(13+\frac{16}{\kappa_{m}^{2}+1}-\frac{9}{\left(\kappa_{m}^{2}+1\right)^{2}}\right) R_{m} S_{2}^{\prime}(0)\right] R_{m} R_{0}^{\prime \prime}(0) \frac{R_{m}^{2} e^{\prime \prime}(0)}{\kappa_{m}} \\
& -1440\left(6 \kappa_{m}^{2}-41-\frac{228}{\kappa_{m}^{2}+1}+\frac{72}{\left(\kappa_{m}^{2}+1\right)^{2}}\right) R_{m} A_{2}^{\prime}(0) \frac{R_{m} Z_{0}^{\prime \prime}(0)}{\kappa_{m}} \frac{R_{m}^{2} e^{\prime \prime}(0)}{\kappa_{m}}
\end{aligned}
$$




$$
\begin{aligned}
& -60\left[3\left(6 \kappa_{m}^{2}+19-\frac{26}{\kappa_{m}^{2}+1}+\frac{12}{\left(\kappa_{m}^{2}+1\right)^{2}}+4\left(27 \kappa_{m}^{2}+21-\frac{50}{\kappa_{m}^{2}+1}+\frac{36}{\left(\kappa_{m}^{2}+1\right)^{2}}\right) R_{m} S_{2}^{\prime}(0)\right)\right. \\
& +6\left(29 \kappa_{m}^{2}-59+\frac{52}{\kappa_{m}^{2}+1}+\frac{72}{\left(\kappa_{m}^{2}+1\right)^{2}}+8\left(55 \kappa_{m}^{2}+319+\frac{172}{\kappa_{m}^{2}+1}-\frac{120}{\left(\kappa_{m}^{2}+1\right)^{2}}\right) R_{m} S_{2}^{\prime}(0)\right) R_{m}^{2} S_{2}^{\prime}(0)^{2} \\
& -6\left(17 \kappa_{m}^{2}+25-\frac{316}{\kappa_{m}^{2}+1}+\frac{168}{\left(\kappa_{m}^{2}+1\right)^{2}}-8\left(33 \kappa_{m}^{2}+285+\frac{340}{\kappa_{m}^{2}+1}-\frac{168}{\left(\kappa_{m}^{2}+1\right)^{2}}\right) R_{m} S_{2}^{\prime}(0)\right) R_{m}^{2} A_{2}^{\prime}(0)^{2} \\
& \left.+4\left(3 \kappa_{m}^{2}+19+\frac{8}{\kappa_{m}^{2}+1}\right) R_{m}^{3} S_{2}^{(3)}(0)\right] R_{m} R_{0}^{\prime \prime}(0)-16\left[1 8 0 \left(5 \kappa_{m}^{2}+16-\frac{82}{\kappa_{m}^{2}+1}+\frac{48}{\left(\kappa_{m}^{2}+1\right)^{2}}\right.\right. \\
& \left.-4\left(7 \kappa_{m}^{2}-127-\frac{176}{\kappa_{m}^{2}+1}+\frac{60}{\left(\kappa_{m}^{2}+1\right)^{2}}\right) R_{m} S_{2}^{\prime}(0)\right) R_{m} A_{2}^{\prime}(0) R_{m} S_{2}^{\prime}(0) \\
& -45\left(3 \kappa_{m}^{2}-117+\frac{136}{\kappa_{m}^{2}+1}-\frac{48}{\left(\kappa_{m}^{2}+1\right)^{2}}+64\left(\kappa_{m}^{2}-20-\frac{71}{\kappa_{m}^{2}+1}+\frac{21}{\left(\kappa_{m}^{2}+1\right)^{2}}\right) R_{m}^{2} A_{2}^{\prime}(0)^{2}\right) R_{m} A_{2}^{\prime}(0) \\
& \left.-30\left(2 \kappa_{m}^{2}-13-\frac{12}{\kappa_{m}^{2}+1}\right) R_{m}^{3} A_{2}^{(3)}(0)\right] \frac{R_{m} Z_{0}^{\prime \prime}(0)}{\kappa_{m}}-90\left[11 \kappa_{m}^{2}+83-\frac{136}{\kappa_{m}^{2}+1}+\frac{72}{\left(\kappa_{m}^{2}+1\right)^{2}}\right. \\
& -\left(4\left(\kappa_{m}^{2}+37+\frac{56}{\kappa_{m}^{2}+1}-\frac{72}{\left(\kappa_{m}^{2}+1\right)^{2}}\right)-8\left(22 \kappa_{m}^{2}+359+\frac{332}{\kappa_{m}^{2}+1}-\frac{180}{\left(\kappa_{m}^{2}+1\right)^{2}}\right) R_{m} S_{2}^{\prime}(0)\right) R_{m} S_{2}^{\prime}(0) \\
& \left.+8\left(16 \kappa_{m}^{2}+291+\frac{612}{\kappa_{m}^{2}+1}-\frac{252}{\left(\kappa_{m}^{2}+1\right)^{2}}\right) R_{m}^{2} A_{2}^{\prime}(0)^{2}\right] \frac{R_{m}^{2} e^{\prime \prime}(0)}{\kappa_{m}}-720\left[5 \kappa_{m}^{2}-5-\frac{6}{\kappa_{m}^{2}+1}+\frac{12}{\left(\kappa_{m}^{2}+1\right)^{2}}\right. \\
& -\left(2\left(14 \kappa_{m}^{2}+40-\frac{49}{\kappa_{m}^{2}+1}+\frac{18}{\left(\kappa_{m}^{2}+1\right)^{2}}\right)+\left(2\left(\kappa_{m}^{2}-55-\frac{52}{\kappa_{m}^{2}+1}+\frac{120}{\left(\kappa_{m}^{2}+1\right)^{2}}\right)\right.\right. \\
& \left.\left.\left.+\left(425 \kappa_{m}^{2}+2063+\frac{860}{\kappa_{m}^{2}+1}-\frac{600}{\left(\kappa_{m}^{2}+1\right)^{2}}\right) R_{m} S_{2}^{\prime}(0)\right) R_{m} S_{2}^{\prime}(0)\right) R_{m} S_{2}^{\prime}(0)\right] R_{m} S_{2}^{\prime}(0) \\
& +720\left[7 \kappa_{m}^{2}+141-\frac{170}{\kappa_{m}^{2}+1}+\frac{84}{\left(\kappa_{m}^{2}+1\right)^{2}}-2\left(7 \kappa_{m}^{2}-1+\frac{172}{\kappa_{m}^{2}+1}-\frac{168}{\left(\kappa_{m}^{2}+1\right)^{2}}\right.\right. \\
& \left.\left.-\left(223 \kappa_{m}^{2}+2209+\frac{1844}{\kappa_{m}^{2}+1}-\frac{840}{\left(\kappa_{m}^{2}+1\right)^{2}}\right) R_{m} S_{2}^{\prime}(0)\right) R_{m} S_{2}^{\prime}(0)+\left(97 \kappa_{m}^{2}+2055+\frac{3324}{\kappa_{m}^{2}+1}-\frac{1176}{\left(\kappa_{m}^{2}+1\right)^{2}}\right) R_{m}^{2} A_{2}^{\prime}(0)^{2}\right] R_{m}^{2} A_{2}^{\prime}(0)^{2} \\
& -240\left[2 \kappa_{m}^{2}+7+\frac{4}{\kappa_{m}^{2}+1}-32\left(\kappa_{m}^{2}+5+\frac{2}{\kappa_{m}^{2}+1}\right) R_{m} S_{2}^{\prime}(0)\right] R_{m}^{3} S_{2}^{(3)}(0)+960\left(5 \kappa_{m}^{2}+41+\frac{24}{\kappa_{m}^{2}+1}\right) R_{m} A_{2}^{\prime}(0) R_{m}^{3} A_{2}^{(3)}(0) \\
& +720\left[2\left(10 \kappa_{m}^{2}-19+\frac{20}{\kappa_{m}^{2}+1}\right)+\left(39 \kappa_{m}^{2}+5+\frac{40}{\kappa_{m}^{2}+1}\right) R_{m}^{2} R_{0}^{\prime \prime}(0)^{2}+\left(41 \kappa_{m}^{2}+91-\frac{40}{\kappa_{m}^{2}+1}\right) \frac{R_{m}^{2} Z_{0}^{\prime \prime}(0)^{2}}{\kappa_{m}^{2}}\right. \\
& +4\left(3 \kappa_{m}^{2}-3+\frac{10}{\kappa_{m}^{2}+1}-\left(37 \kappa_{m}^{2}+157-\frac{40}{\kappa_{m}^{2}+1}\right) R_{m} S_{2}^{\prime}(0)\right) R_{m} R_{0}^{\prime \prime}(0) \\
& +8\left(15 \kappa_{m}^{2}-68+\frac{60}{\kappa_{m}^{2}+1}\right) R_{m} A_{2}^{\prime}(0) \frac{R_{m} \mathrm{Z}_{0}^{\prime \prime}(0)}{\kappa_{m}}-\left(117+13 \kappa_{m}^{2}-\frac{96}{\kappa_{m}^{2}+1}\right) \frac{R_{m}^{2} e^{\prime \prime}(0)}{\kappa_{m}} \\
& -2\left(111+11 \kappa_{m}^{2}-\frac{40}{\kappa_{m}^{2}+1}-\left(262 \kappa_{m}^{2}+810-\frac{144}{\kappa_{m}^{2}+1}\right) R_{m} S_{2}^{\prime}(0)\right) R_{m} S_{2}^{\prime}(0) \\
& \left.+4\left(65 \kappa_{m}^{2}+255-\frac{248}{\kappa_{m}^{2}+1}\right) R_{m}^{2} A_{2}^{\prime}(0)^{2}+4\left(41 \kappa_{m}^{2}+51\right) \frac{R_{m}^{2} S_{3}^{\prime}(0)}{a}\right] \frac{R_{m}^{2} S_{3}^{\prime}(0)}{a}
\end{aligned}
$$




$$
\begin{aligned}
& -1440\left[6\left(2 \kappa_{m}^{2}-1-\frac{4}{\kappa_{m}^{2}+1}\right) R_{m} R_{0}^{\prime \prime}(0) \frac{R_{m} Z_{0}^{\prime \prime}(0)}{\kappa_{m}}+8\left(3 \kappa_{m}^{2}+14+\frac{4}{\kappa_{m}^{2}+1}\right) R_{m} A_{2}^{\prime}(0) R_{m} R_{0}^{\prime \prime}(0)\right. \\
& +2\left(2 \kappa_{m}^{2}-1-\frac{4}{\kappa_{m}^{2}+1}+4\left(2 \kappa_{m}^{2}+17+\frac{8}{\kappa_{m}^{2}+1}\right) R_{m} S_{2}^{\prime}(0)\right) \frac{R_{m} Z_{0}^{\prime \prime}(0)}{\kappa_{m}} \\
& \left.+\left(7 \kappa_{m}^{2}+53+\frac{32}{\kappa_{m}^{2}+1}-36\left(\kappa_{m}^{2}+13+\frac{8}{\kappa_{m}^{2}+1}\right) R_{m} S_{2}^{\prime}(0)\right) R_{m} A_{2}^{\prime}(0)-4\left(7 \kappa_{m}^{2}+19\right) \frac{R_{m}^{2} A_{3}^{\prime}(0)}{a}\right] \frac{R_{m}^{2} A_{3}^{\prime}(0)}{a} \\
& +480\left(5 \kappa_{m}^{2}+11\right) \frac{R_{m}^{4} S_{3}^{(3)}(0)}{a}+4\left(\kappa_{m}^{2}-1\right)\left(\frac{R_{m}^{4} I_{T}^{(6)}(0)}{I_{T}^{\prime \prime}(0)}\right)+5\left(\kappa_{m}^{2}-1\right)\left(\frac{R_{m}^{4} I_{T}^{(4)}(0)^{2}}{I_{T}^{\prime \prime}(0)^{2}}\right) \\
& -30\left[\kappa_{m}^{2}-9+\frac{8}{\kappa_{m}^{2}+1}+2\left(3 \kappa_{m}^{2}-2+\frac{4}{\kappa_{m}^{2}+1}\right) R_{m}^{2} R_{0}^{\prime \prime}(0)^{2}+2\left(4 \kappa_{m}^{2}+19-\frac{4}{\kappa_{m}^{2}+1}\right) \frac{R_{m}^{2} Z_{0}^{\prime \prime}(0)^{2}}{\kappa_{m}^{2}}\right. \\
& -2\left(2 \kappa_{m}^{2}+5-\frac{4}{\kappa_{m}^{2}+1}+32\left(\kappa_{m}^{2}+2-\frac{1}{\kappa_{m}^{2}+1}\right) R_{m} S_{2}^{\prime}(0)\right) R_{m} R_{0}^{\prime \prime}(0) \\
& +8\left(\kappa_{m}^{2}-41+\frac{16}{\kappa_{m}^{2}+1}\right) R_{m} A_{2}^{\prime}(0) \frac{R_{m} Z_{0}^{\prime \prime}(0)}{\kappa_{m}}+\left(\kappa_{m}^{2}-31+\frac{24}{\kappa_{m}^{2}+1}\right) \frac{R_{m}^{2} e^{\prime \prime}(0)}{\kappa_{m}} \\
& -4\left(3 \kappa_{m}^{2}+1-\frac{8}{\kappa_{m}^{2}+1}-2\left(14 \kappa_{m}^{2}+35-\frac{20}{\kappa_{m}^{2}+1}\right) R_{m} S_{2}^{\prime}(0)\right) R_{m} S_{2}^{\prime}(0) \\
& \left.+8\left(4 \kappa_{m}^{2}+55-\frac{28}{\kappa_{m}^{2}+1}\right) R_{m}^{2} A_{2}^{\prime}(0)^{2}+56\left(\kappa_{m}^{2}-1\right) \frac{R_{m}^{2} S_{3}^{\prime}(0)}{a}\right]\left(\frac{R_{m}^{2} I_{T}^{(4)}(0)}{I_{T}^{\prime \prime}(0)}\right) \\
& -30 \kappa_{m}^{2}\left[1-4\left(R_{m} R_{0}^{\prime \prime}(0)-2 R_{m} S_{2}^{\prime}(0)\right)\right]\left(\frac{8 \pi^{2}\left(\kappa_{m}^{2}+1\right)^{2} R_{m}^{2} p^{(4)}(0)}{\kappa_{m}^{2} \mu_{0} I_{T}^{\prime \prime}(0)^{2}}\right) \\
& -30 \kappa_{m}^{2}\left[3-4 R_{m}^{3} R_{0}^{(4)}(0)-12\left(R_{m} R_{0}^{\prime \prime}(0)-\frac{R_{m}^{2} e^{\prime \prime}(0)}{\kappa_{m}}-4 R_{m} S_{2}^{\prime}(0)\right) R_{m} R_{0}^{\prime \prime}(0)-3\left(1+8 R_{m} S_{2}^{\prime}(0)\right) \frac{R_{m}^{2} e^{\prime \prime}(0)}{\kappa_{m}}\right. \\
& -12\left(1+4 R_{m} S_{2}^{\prime}(0)\right) R_{m} S_{2}^{\prime}(0)+16 R_{m}^{3} S_{2}^{(3)}(0) \\
& \left.-24\left(\left(1-8 R_{m} S_{2}^{\prime}(0)\right) \frac{R_{m}^{2} S_{3}^{\prime}(0)}{a}-8 R_{m} A_{2}^{\prime}(0) \frac{R_{m}^{2} A_{3}^{\prime}(0)}{a}\right)\right]\left(\frac{8 \pi^{2}\left(1+\kappa_{m}^{2}\right)^{2} p^{\prime \prime}(0)}{\kappa_{m}^{2} \mu_{0} I_{T}^{\prime \prime}(0)^{2}}\right)=0 .
\end{aligned}
$$

Next to the lowest order term for the symmetric triangularity coefficient $S_{2}(\rho)$ :

$$
\begin{aligned}
& 3\left(67 \kappa_{m}^{2}-11+\frac{32}{\kappa_{m}^{2}+1}\right)-8\left(\kappa_{m}^{2}-3\right) R_{m}^{3} R_{0}^{(4)}(0)+12\left(43 \kappa_{m}^{2}-3+\frac{16}{\kappa_{m}^{2}+1}\right) R_{m}^{3} R_{0}^{\prime \prime}(0)^{3} \\
& +12\left(31 \kappa_{m}^{2}+73-\frac{16}{\kappa_{m}^{2}+1}\right) R_{m} R_{0}^{\prime \prime}(0) \frac{R_{m}^{2} Z_{0}^{\prime \prime}(0)^{2}}{\kappa_{m}^{2}}+\left[435 \kappa_{m}^{2}-363+\frac{288}{\kappa_{m}^{2}+1}-48\left(21 \kappa_{m}^{2}+131-\frac{60}{\kappa_{m}^{2}+1}\right) R_{m} S_{2}^{\prime}(0)\right] R_{m}^{2} R_{0}^{\prime \prime}(0)^{2} \\
& +3\left[47 \kappa_{m}^{2}+89-\frac{32}{\kappa_{m}^{2}+1}-16\left(25 \kappa_{m}^{2}+83+\frac{28}{\kappa_{m}^{2}+1}\right) R_{m} S_{2}^{\prime}(0)\right] \frac{R_{m}^{2} Z_{0}^{\prime \prime}(0)^{2}}{\kappa_{m}^{2}} \\
& +48\left(35 \kappa_{m}^{2}-139+\frac{64}{\kappa_{m}^{2}+1}\right) R_{m} A_{2}^{\prime}(0) R_{m} R_{0}^{\prime \prime}(0) \frac{R_{m} Z_{0}^{\prime \prime}(0)}{\kappa_{m}}+6\left(21 \kappa_{m}^{2}-221+\frac{96}{\kappa_{m}^{2}+1}\right) R_{m} R_{0}^{\prime \prime}(0) \frac{R_{m}^{2} e^{\prime \prime}(0)}{\kappa_{m}} \\
& +6\left[111 \kappa_{m}^{2}-39+\frac{48}{\kappa_{m}^{2}+1}+8\left(2\left(4 \kappa_{m}^{2}-31+\frac{30}{\kappa_{m}^{2}+1}\right)+\left(113 \kappa_{m}^{2}+407+\frac{144}{\kappa_{m}^{2}+1}\right) R_{m} S_{2}^{\prime}(0)\right) R_{m} S_{2}^{\prime}(0)\right. \\
& \left.+8\left(45 \kappa_{m}^{2}+307-\frac{112}{\kappa_{m}^{2}+1}\right) R_{m}^{2} A_{2}^{\prime}(0)^{2}\right] R_{m} R_{0}^{\prime \prime}(0)+24\left[29 \kappa_{m}^{2}-105+\frac{64}{\kappa_{m}^{2}+1}-8\left(5 \kappa_{m}^{2}-59-\frac{112}{\kappa_{m}^{2}+1}\right) R_{m} S_{2}^{\prime}(0)\right] \\
& \times R_{m} A_{2}^{\prime}(0) \frac{R_{m} Z_{0}^{\prime \prime}(0)}{\kappa_{m}}+6\left[\kappa_{m}^{2}-97+\frac{48}{\kappa_{m}^{2}+1}+12\left(33+\kappa_{m}^{2}+\frac{56}{\kappa_{m}^{2}+1}\right) R_{m} S_{2}^{\prime}(0)\right] \frac{R_{m}^{2} e^{\prime \prime}(0)}{\kappa_{m}} \\
& -12\left[2\left(13 \kappa_{m}^{2}+123-\frac{72}{\kappa_{m}^{2}+1}\right)\right.
\end{aligned}
$$




$$
\begin{aligned}
& \left.-\left(87 \kappa_{m}^{2}+289+\frac{288}{\kappa_{m}^{2}+1}-16\left(57 \kappa_{m}^{2}+227+\frac{140}{\kappa_{m}^{2}+1}\right) R_{m} S_{2}^{\prime}(0)\right) R_{m} S_{2}^{\prime}(0)\right] R_{m} S_{2}^{\prime}(0) \\
& +12\left[97 \kappa_{m}^{2}+471-\frac{224}{\kappa_{m}^{2}+1}-32\left(19 \kappa_{m}^{2}+121+\frac{98}{\kappa_{m}^{2}+1}\right) R_{m} S_{2}^{\prime}(0)\right] R_{m}^{2} A_{2}^{\prime}(0)^{2} \\
& -48\left(3 \kappa_{m}^{2}+7\right) R_{m}^{3} S_{2}^{(3)}(0)+12\left[101 \kappa_{m}^{2}+119+2\left(89 \kappa_{m}^{2}+99\right) R_{m} R_{0}^{\prime \prime}(0)-4\left(145 \kappa_{m}^{2}+387\right) R_{m} S_{2}^{\prime}(0)\right] \frac{R_{m}^{2} S_{3}^{\prime}(0)}{a} \\
& +24\left[\left(17 \kappa_{m}^{2}+43\right) \frac{R_{m} Z_{0}^{\prime \prime}(0)}{\kappa_{m}}-10\left(\kappa_{m}^{2}+19\right) R_{m} A_{2}^{\prime}(0)\right] \frac{R_{m}^{2} A_{3}^{\prime}(0)}{a} \\
& -16\left[\kappa_{m}^{2}+2\left(2 \kappa_{m}^{2}+1\right) R_{m} R_{0}^{\prime \prime}(0)-\left(\kappa_{m}^{2}+15\right) R_{m} S_{2}^{\prime}(0)\right]\left(\frac{R_{m}^{2} I_{T}^{(4)}(0)}{I_{T}^{\prime \prime}(0)}\right) \\
& +16 \kappa_{m}^{2}\left(\frac{8 \pi^{2}\left(\kappa_{m}^{2}+1\right)^{2} R_{m}^{2} p^{(4)}(0)}{\kappa_{m}^{2} \mu_{0} I_{T}^{\prime \prime}(0)^{2}}\right)+48 \kappa_{m}^{2}\left[\left(1-4 R_{m} S_{2}^{\prime}(0)\right)\left(R_{m} R_{0}^{\prime \prime}(0)-R_{m} S_{2}^{\prime}(0)\right)\right. \\
& \left.+\frac{R_{m}^{2} e^{\prime \prime}(0)}{\kappa_{m}}-4 R_{m}^{2} A_{2}^{\prime}(0)^{2}\right]\left(\frac{8 \pi^{2}\left(\kappa_{m}^{2}+1\right)^{2} p^{\prime \prime}(0)}{\kappa_{m}^{2} \mu_{0} I_{T}^{\prime \prime}(0)^{2}}\right)=0 .
\end{aligned}
$$

Next to the lowest order term for the antisymmetric triangularity coefficient $A_{2}(\rho)$ :

$$
\begin{aligned}
& 8\left(\kappa_{m}^{2}-2\right) \frac{R_{m}^{3} Z_{0}^{(4)}(0)}{\kappa_{m}}+6\left[\left(9 \kappa_{m}^{2}-49+\frac{32}{\kappa_{m}^{2}+1}\right) R_{m}^{2} R_{0}^{\prime \prime}(0)^{2}-\left(3 \kappa_{m}^{2}+53+\frac{32}{\kappa_{m}^{2}+1}\right) \frac{R_{m}^{2} Z_{0}^{\prime \prime}(0) 2}{\kappa_{m}^{2}}\right] \frac{R_{m} Z_{0}^{\prime \prime}(0)}{\kappa_{m}} \\
& +48\left[\left(3 \kappa_{m}^{2}+41-\frac{26}{\kappa_{m}^{2}+1}\right) R_{m}^{2} R_{0}^{\prime \prime}(0)^{2}-2\left(\kappa_{m}^{2}-24-\frac{45}{\kappa_{m}^{2}+1}\right) \frac{R_{m}^{2} Z_{0}^{\prime \prime}(0)^{2}}{\kappa_{m}^{2}}\right] R_{m} A_{2}^{\prime}(0) \\
& +3\left[5 \kappa_{m}^{2}-13+\frac{64}{\kappa_{m}^{2}+1}-8\left(15 \kappa_{m}^{2}-31-\frac{64}{\kappa_{m}^{2}+1}\right) R_{m} S_{2}^{\prime}(0)\right] R_{m} R_{0}^{\prime \prime}(0) \frac{R_{m} Z_{0}^{\prime \prime}(0)}{\kappa_{m}} \\
& -3\left(19 \kappa_{m}^{2}-99-\frac{192}{\kappa_{m}^{2}+1}\right) \frac{R_{m} Z_{0}^{\prime \prime}(0)}{\kappa_{m}} \frac{R_{m}^{2} e^{\prime \prime}(0)}{\kappa_{m}}+48\left[\kappa_{m}^{2}+12-\frac{26}{\kappa_{m}^{2}+1}-4\left(3 \kappa_{m}^{2}+8+\frac{52}{\kappa_{m}^{2}+1}\right) R_{m} S_{2}^{\prime}(0)\right] R_{m} A_{2}^{\prime}(0) R_{m} R_{0}^{\prime \prime}(0) \\
& -3\left[17 \kappa_{m}^{2}+111-\frac{64}{\kappa_{m}^{2}+1}+8\left(9 \kappa_{m}^{2}+223+\frac{1056}{\kappa_{m}^{2}+1}\right) R_{m}^{2} A_{2}^{\prime}(0)^{2}\right. \\
& \left.+4\left(9 \kappa_{m}^{2}+19-\frac{64}{\kappa_{m}^{2}+1}+2\left(\kappa_{m}^{2}+239+\frac{160}{\kappa_{m}^{2}+1}\right) R_{m} S_{2}^{\prime}(0)\right) R_{m} S_{2}^{\prime}(0)\right] \frac{R_{m} Z_{0}^{\prime \prime}(0)}{\kappa_{m}}-12\left(13 \kappa_{m}^{2}+21+\frac{312}{\kappa_{m}^{2}+1}\right) R_{m} A_{2}^{\prime}(0) \frac{R_{m}^{2} e^{\prime \prime}(0)}{\kappa_{m}} \\
& -12\left[13 \kappa_{m}^{2}-189+\frac{416}{\kappa_{m}^{2}+1}-8\left(17 \kappa_{m}^{2}+219+\frac{260}{\kappa_{m}^{2}+1}\right) R_{m} S_{2}^{\prime}(0)\right] R_{m} S_{2}^{\prime}(0) R_{m} A_{2}^{\prime}(0) \\
& -12\left[15 \kappa_{m}^{2}-167+\frac{104}{\kappa_{m}^{2}+1}-16\left(5 \kappa_{m}^{2}+99+\frac{182}{\kappa_{m}^{2}+1}\right) R_{m}^{2} A_{2}^{\prime}(0)^{2}\right] R_{m} A_{2}^{\prime}(0) \\
& +8\left(7 \kappa_{m}^{2}+23\right) R_{m}^{3} A_{2}^{(3)}(0)+12\left[\left(11 \kappa_{m}^{2}-79\right) \frac{R_{m} Z_{0}^{\prime \prime}(0)}{\kappa_{m}}+2\left(69 \kappa_{m}^{2}+247\right) R_{m} A_{2}^{\prime}(0)\right] \frac{R_{m}^{2} S_{3}^{\prime}(0)}{a} \\
& -6\left[15 \kappa_{m}^{2}+77+\left(38 \kappa_{m}^{2}+82\right) R_{m} R_{0}^{\prime \prime}(0)-4\left(7 \kappa_{m}^{2}+109\right) R_{m} S_{2}^{\prime}(0)\right] \frac{R_{m}^{2} A_{3}^{\prime}(0)}{a} \\
& +8\left[\left(\kappa_{m}^{2}+5\right) \frac{R_{m} Z_{0}^{\prime \prime}(0)}{\kappa_{m}}+\left(5 \kappa_{m}^{2}-21\right) R_{m} A_{2}^{\prime}(0)\right]\left(\frac{R_{m}^{2} I_{T}^{(4)}(0)}{I_{T}^{\prime \prime}(0)}\right) \\
& -24 \kappa_{m}^{2}\left[\left(1-4\left(R_{m} R_{0}^{\prime \prime}(0)-2 R_{m} S_{2}^{\prime}(0)\right)\right) R_{m} A_{2}^{\prime}(0)+4 \frac{R_{m}^{2} A_{3}^{\prime}(0)}{a}\right]\left(\frac{8 \pi^{2}\left(\kappa_{m}^{2}+1\right)^{2} p^{\prime \prime}(0)}{\kappa_{m}^{2} \mu_{0} I_{T}^{\prime \prime}(0)^{2}}\right)=0 .
\end{aligned}
$$

The lowest order term for the symmetric quadrangularity coefficient $S_{3}(\rho)$ : 


$$
\begin{gathered}
7\left(5 \kappa_{m}^{2}+3\right)+\left[\left(57 \kappa_{m}^{2}+47\right) R_{m} R_{0}^{\prime \prime}(0)+2\left(13 \kappa_{m}^{2}+7-2\left(43 \kappa_{m}^{2}+141\right) R_{m} S_{2}^{\prime}(0)\right)\right] R_{m} R_{0}^{\prime \prime}(0) \\
+\left[\left(47 \kappa_{m}^{2}+57\right) \frac{R_{m} Z_{0}^{\prime \prime}(0)}{\kappa_{m}}-4\left(71-47 \kappa_{m}^{2}\right) R_{m} A_{2}^{\prime}(0)\right] \frac{R_{m} Z_{0}^{\prime \prime}(0)}{\kappa_{m}}-14\left(\kappa_{m}^{2}+7\right) \frac{R_{m}^{2} e^{\prime \prime}(0)}{\kappa_{m}} \\
-2\left[17\left(\kappa_{m}^{2}+7\right)-2\left(217 \kappa_{m}^{2}+495\right) R_{m} S_{2}^{\prime}(0)\right] R_{m} S_{2}^{\prime}(0)+28\left(17 \kappa_{m}^{2}+39\right) R_{m}^{2} A_{2}^{\prime}(0)^{2} \\
+616\left(\kappa_{m}^{2}+1\right) \frac{R_{m}^{2} S_{3}^{\prime}(0)}{a}+16 \kappa_{m}^{2}\left[R_{m} R_{0}^{\prime \prime}(0)-4 R_{m} S_{2}^{\prime}(0)\right]\left(\frac{8 \pi^{2}\left(\kappa_{m}^{2}+1\right)^{2} p^{\prime \prime}(0)}{\kappa_{m}^{2} \mu_{0} I_{T}^{\prime \prime}(0)^{2}}\right)=0 .
\end{gathered}
$$

The lowest order term for the antisymmetric quadrangularity coefficient $A_{3}(\rho)$ :

$$
\begin{aligned}
& {\left[7\left(\kappa_{m}^{2}-1\right)\left(1+3 R_{m} R_{0}^{\prime \prime}(0)\right)+2\left(13 \kappa_{m}^{2}+67\right) R_{m} S_{2}^{\prime}(0)\right] \frac{R_{m} Z_{0}^{\prime \prime}(0)}{\kappa_{m}}} \\
& \quad+\left[19 \kappa_{m}^{2}+77+10\left(5 \kappa_{m}^{2}+11\right) R_{m} R_{0}^{\prime \prime}(0)-4\left(23 \kappa_{m}^{2}+137\right) R_{m} S_{2}^{\prime}(0)\right] R_{m} A_{2}^{\prime}(0) \\
& \quad-140\left(\kappa_{m}^{2}+1\right) \frac{R_{m}^{2} A_{3}^{\prime}(0)}{a}+16 \kappa_{m}^{2} R_{m} A_{2}^{\prime}(0)\left(\frac{8 \pi^{2}\left(\kappa_{m}^{2}+1\right)^{2} p^{\prime \prime}(0)}{\kappa_{m}^{2} \mu_{0} I_{T}^{\prime \prime}(0)^{2}}\right)=0 .
\end{aligned}
$$

${ }^{1}$ G. O. Ludwig, "Analytical solution of the tokamak equilibrium. II. The free-boundary case," Phys. Plasma 24, 092503 (2017).

${ }^{2}$ V. D. Shafranov, "On magnetohydrodynamical equilibrium configurations," Zh. Eksp. Teor. Fiz. 33, 710-722 (1957) [Sov. Phys. JETP 6, 545-554 (1958)].

${ }^{3}$ R. Lüst and A. Schlüter, "Axialsymmetrische magnetohydrodynamische gleichgewichtskonfigurationen," Z. Naturforsch. 12A, 850-854 (1957).

${ }^{4} \mathrm{H}$. Grad and H. Rubin, 1958 "Hydromagnetic equilibria and force-free fields," in Proceedings of the Second United Nations International Conference on the Peaceful Uses of Atomic Energy (United Nations, Geneva, 1958), Vol. 31, pp. 190-197.

${ }^{5} \mathrm{~L}$. S. Solov'ev, "The theory of hydromagnetic stability of toroidal plasma configurations," Zh. Eksp. Teor. Fiz. 53, 626-643 (1967) [Sov. Phys. JETP 26, 400-407 (1968)].

${ }^{6} \mathrm{E}$. K. Maschke, "Exact solutions of the MHD equilibrium equation for a toroidal plasma," Plasma Phys. 15, 535-541 (1973).

${ }^{7}$ J. P. Sudano, "Equilibrium of a toroidal plasma," Phys. Fluids 17, 1915-1916 (1974).

${ }^{8}$ E. Mazzucato, "Exact equilibria of axisymmetric magnetic configurations," Phys. Fluids 18, 536-540 (1975).

${ }^{9}$ S. B. Zheng, A. J. Wootton, and E. R. Solano, "Analytical tokamak equilibrium for shaped plasmas," Phys. Plasmas 3, 1176-1178 (1996).

${ }^{10} \mathrm{P}$. J. McCarthy, "Analytic solutions to the Grad-Shafranov equation for tokamak equilibrium with dissimilar source functions," Phys. Plasmas 6, 3554-3560 (1999).

${ }^{11}$ C. V. Atanasiu, S. Günter, K. Lackner, and I. G. Miron, "Analytical solutions to the Grad-Shafranov equation," Phys. Plasmas 11, 3510-3518 (2004).

${ }^{12}$ L. Guazzotto and J. P. Freidberg, "A family of analytic equilibrium solutions for the Grad-Shafranov equation," Phys. Plasmas 14, 112508 (2007).

${ }^{13}$ B. Shi, "Exact single-null diverted tokamak equilibria," Plasma Phys. Controlled Fusion 51, 105008-105012 (2009).
${ }^{14}$ R. Srinivasan, L. L. Lao, and M. S. Chu, "Analytical description of poloidally diverted tokamak equilibrium with linear stream functions," Plasma Phys. Controlled Fusion 52, 035007-035012 (2010).

${ }^{15}$ H. Weitzner, 1981 Appendix in Ref. 18.

${ }^{16} \mathrm{G}$. O. Ludwig, "Direct variational solutions of the tokamak equilibrium problem," Plasma Phys. Controlled Fusion 39, 2021-2037 (1997).

${ }^{17}$ V. A. Yavorskij, K. Schoepf, Z. N. Andrushchenko, B. H. Cho, V. Y. Goloborod'ko, and S. N. Reznyk, "Analytical models of axisymmetric toroidal magnetic fields with non-circular flux surfaces," Plasma Phys. Controlled Fusion 43, 249-269 (2001).

${ }^{18}$ L. L. Lao, S. P. Hirshmann, and R. M. Wieland, "Variational moment solutions to the Grad-Shafranov equation," Phys. Fluids 24, 1431-1441 (1981).

${ }^{19}$ G. O. Ludwig, "Direct variational solutions to the Grad-SchlüterShafranov equation," Plasma Phys. Controlled Fusion 37, 633-646 (1995).

${ }^{20}$ L. E. Zakharov and V. D. Shafranov, "Equilibrium of current-carrying plasmas in toroidal configurations," Rev. Plasma Phys. 11, 153-308 (1986).

${ }^{21}$ G. O. Ludwig, P. Rodrigues, and J. P. S. Bizarro, "Tokamak equilibria with strong toroidal current density reversal," Nucl. Fusion 53, 053001 (2013).

${ }^{22}$ M. D. Kruskal and R. M. Kulsrud, "Equilibrium of a magnetically confined plasma in a toroid," Phys. Fluids 1, 265-274 (1958).

${ }^{23} \mathrm{H}$. Grad, "Some new variational properties of hydromagnetic equilibria," Phys. Fluids 7, 1283-1292 (1964).

${ }^{24} \mathrm{~V}$. D. Khait, "Variational method for approximately solving the problem of the MHD equilibrium of a tokamak plasma," Sov. J. Plasma Phys. 6, 476-478 (1980).

${ }^{25}$ M. Shimada et al., "Overview and summary," Nucl. Fusion 47, S1-S17 (2007).

${ }^{26} \mathrm{E}$. Minardi, "The relation between current density and pressure in the Ohmic tokamak," Phys. Lett. A 240, 70-72 (1998). 